\title{
Removal of pharmaceutical compounds in water and wastewater using fungal oxidoreductase enzymes ${ }^{\varkappa}$
}

\author{
Mitra Naghdi ${ }^{\mathrm{a}}$, Mehrdad Taheran ${ }^{\mathrm{a}}$, Satinder Kaur Brar ${ }^{\mathrm{a}, *}$, Azadeh Kermanshahi-pour ${ }^{\mathrm{b}}$, Mausam Verma ${ }^{\mathrm{a}}$, \\ R.Y. Surampallic \\ ${ }^{a}$ INRS-ETE, Université du Québec, 490, Rue de la Couronne, Québec G1K 9A9, Canada

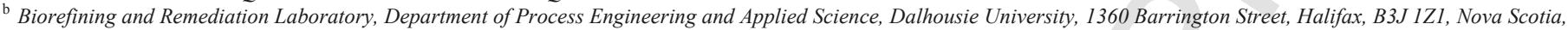 \\ Canada \\ ${ }^{\mathrm{c}}$ Department of Civil Engineering, University of Nebraska-Lincoln, N104 SEC PO Box 886105, Lincoln, NE 68588-6105, USA
}

\section{A R T I C L E IN F O}

\section{Article history:}

Received 29 August 2017

Received in revised form 13 November 2017

Accepted 16 November 2017

Available online $\mathrm{xxx}$

Keywords:

Enzymatic treatment

Pharmaceutical compounds

\begin{abstract}
A B S T R A C T
Due to recalcitrance of some pharmaceutically active compounds (PhACs), conventional wastewater treatment is not able to remove them effectively. Therefore, their occurrence in surface water and potential environmental impact has raised serious global concern. Biological transformation of these contaminants using white-rot fungi (WRF) and their oxidoreductase enzymes has been proposed as a low cost and environmentally friendly solution for water treatment. The removal performance of PhACs by a fungal culture is dependent on several factors, such as fungal species, the secreted enzymes, molecular structure of target compounds, culture medium composition, etc. In recent 20 years, numerous researchers tried to elucidate the removal mechanisms and the effects of important operational parameters such as temperature and $\mathrm{pH}$ on the enzymatic treatment of PhACs. This review summarizes and analyzes the studies performed on PhACs re-
\end{abstract}

Abbreviation: PhACs, Pharmaceutically active compounds; WRF, white-rot fungi; TPs, transformation products; WWTPs, wastewater treatment plants; AOPs, advanced oxidation processes; ACT, Acetaminophen; AMP, Amitriptyline; ATL, Atenolol; BFB, Bezafibrate; CAF, Caffeine; CBZ, Carbamazepine; CET, Cetirizine; CTC, Chlortetracycline; CPF, Ciprofloxacine; CTL, Citalopram; DZP, Diazepam; DCF, Diclofenac; DC, Doxycycline; EFC, Enrofloxacin; ETM, Erythromycin; FEF, Fenofibrate; FEP, Fenoprofen; FLX, Fluoxetine; GFZ, Gemfibrozil; IBP, Ibuprofen; IDM, Indomethacin; KEP, Ketoprofen; MFA, Mefenamic acid; NPX, Naproxen; NOR, Norfloxacin; OST, Oseltamivir; OTC, Oxytetracycline; PCT, Paracetamol; PPL, Propranolol; PPZ, Propyphenazone; SDM, Sulfadimethoxine; SMZ, Sulfamethazine; SMX, Sulfamethoxazole; SMM, Sulfamonomethoxine; SPY, Sulfapyridine; STZ, Sulfathiazole; SAA, Sulfonamides sulfanilamide; TC, Tetracycline; TMP, Trimethoprim; LiP, lignin peroxidase; MnP, manganese peroxidase; VP, versatile peroxidase; Lac, laccase; VEA, Veratryl alcohol; $\mathrm{K}_{\text {ow }}$, octanol/water partition coefficient; CYP450, cytochrome P450; PC, Phanerochaete chrysosporium; TV, Trametes versicolor; PS, Phanerochaete sordida; PO, Pleurotus ostreatus; DI-MS, direct inlet-mass spectrometry; ${ }^{1} \mathrm{H}$ NMR, ${ }^{1} \mathrm{H}$ nuclear magnetic resonance; LC-ESI-TOF-MS, Liquid chromatography electrospray time-of-flight mass spectrometry; HPLC-DAD-MS, high-performance liquid chromatography-diode array detection-electrospray ionization mass spectrometry; LDTD-MS, laser diode thermal desorption-mass spectroscopy; YES, Yeast Estrogen Screen assay; hER, human estrogen receptor; HRP, horseradish peroxidase; ORP, oxidation reduction potential; EWGs, electron withdrawing groups; SA, syringaldazine; HBT, 1-hydroxybenzotriazole; ABTS, 2,2'-azino-bis(3-ethylbenzothiazoline-6-sulphonic acid); TEMPO, 2,2,6,6-tetramethylpiperidinyloxyl; VLA, violuric acid; MT, Myceliophthora thermophila; MBR, membrane bioreactor; ACE, acetosyringone; EDGs, electron donating groups; EMR, enzymatic membrane reactor; GAC, granular activated carbon; $\mathrm{COD}$, chemical oxygen demand

? This paper has been recommended for acceptance by Klaus Kummerer.

* Corresponding author.

Email address: satinder.brar@ete.inrs.ca (S.K. Brar) 
White-rot fungi Biodegradation Wastewater moval from spiked pure water and real wastewaters using oxidoreductase enzymes and the data related to degradation efficiencies of the most studied compounds. The review also offers an insight into enzymes immobilization, fungal reactors, mediators, degradation mechanisms and transformation products (TPs) of PhACs. In brief, higher hydrophobicity and having electron-donating groups, such as amine and hydroxyl in molecular structure leads to more effective degradation of PhACs by fungal cultures. For recalcitrant compounds, using redox mediators, such as syringaldehyde increases the degradation efficiency, however they may cause toxicity in the effluent and deactivate the enzyme. Immobilization of enzymes on supports can enhance the performance of enzyme in terms of reusability and stability. However, the immobilization strategy should be carefully selected to reduce the cost and enable regeneration. Still, further studies are needed to elucidate the mechanisms involved in enzymatic degradation and the toxicity levels of TPs and also to optimize the whole treatment strategy to have economical and technical competitiveness.

The performance of enzymatic treatment systems including whole cell fungi, crude and purified free enzyme, immobilized enzyme and hybrid treatments were reviewed and compared.

\section{Introduction}

Agricultural and industrial activities result in release of variety of chemicals into the environment, leading to serious concerns over the health and environmental impact of these chemicals and their associated degradation metabolites (Deblonde et al., 2011; Kim et al., 2007). Moreover, population increase and disposal of municipal sewage contributed to the pollution of waterways and almost $70-80 \%$ of rivers and streams around the world carry polluted water (Husain and Husain, 2007). Beside the potential adverse effects on human health, the released pollutants into waterways may have chronic and acute toxicity to the organisms in aquatic ecosystem and may lead to loss of biodiversity and habitats (Alexander et al., 2012).

Pharmaceutically active compounds (PhACs), are among water pollutants that have been frequently detected in the effluents of wastewater treatment plants (WWTPs) (Marco-Urrea et al., 2009). PhACs are widely used as prescription or non-prescription medicines and after their usage, they find their way into wastewater through urine and feces either as intact substances or metabolites (Naghdi et al., 2017). It is a matter of concern that WWTPs are not able to efficiently remove these pollutants due to their persistent nature, resulting in their discharge into surface water (Lienert et al., 2007). Due to the persistence and high lipid solubility of some of the organic pollutants, they can bioaccumulate in the fatty tissues of living organisms (Burkhardt-Holm, 2011). Recently, some evidences have been found that a few pharmaceutical compounds can mobilize towards the food chain, and hence their concentration is increased (Lagesson et al., 2016). The presence of PhACs in waterways may lead to several issues in the environment, such as male fish feminization as a result of exposure to steroidal hormones and development of antibiotic-resistant genes due to released non-metabolized antibiotics into water (De García et al., 2013; Nazaret and Aminov, 2013).

The worldwide annual consumption of PhACs is estimated to be 100,000 tons or more and the trend is increasing due to the diseases and aging population (Kümmerer, 2008). For instance, about 877 tons of diclofenac, listed as 12th best-selling generics in the world (Lonappan et al., 2016), and 942 tons of carbamazepine, listed as 8th bestselling psychiatric drugs worldwide (Mohapatra et al., 2012), were sold in 2007 in 76 countries. A significant portion of these $\mathrm{PhACs}$ are released into the environment in intact or metabolized form. The increasing concern over the accumulation of micropollutants in the aquatic media triggered many research works to evaluate their biodegradation in wastewater treatment systems (Stackelberg et al., 2007). The results implied that unlike traditional wastewater treatment processes, such as conventional activated sludge, recently-developed methods for wastewater treatment including membrane separation, advanced oxidation processes (AOPs) and adsorption onto activated carbon, are able to achieve high efficiency for PhACs removal (Ikehata et al., 2006; Radjenović et al., 2008; Suárez et al., 2008; 
Tadkaew et al., 2011). However, still, challenges remained with these technologies including the formation of more toxic by-products during AOPs (Kosjek et al., 2009), the disposal of the concentrated stream in membrane separation (Westerhoff et al., 2009) and the regeneration of absorbents (Bathen, 2003). Therefore, development of effective treatment processes to remove PhACs from wastewater is always of high importance. In Table 1, the information about physicochemical properties of the most studied compounds has been presented as they are helpful in the prediction of the efficiency of enzymatic treatment. In the following sections, removal efficiencies of PhACs by different forms of the enzyme (whole-cell culture, crude extracts and immobilized) are also discussed.

Biocatalytic conversion is an environmentally benign alternative method, which involves the use of living organisms or their enzymes. This treatment method requires lower energy input, works under moderate conditions and produces less or no toxic by-products compared to other conventional technologies (Asif et al., 2017). Additionally, the specificity of enzymes towards substrate facilitates minimizing the unfavorable side reactions, where required (Senthivelan et al., 2016). Therefore, enzymes are promising options for the selective removal of pollutants from water and wastewater (Demarche et al., 2012). In recent years, numerous researchers studied the treatment of wastewater with the enzymatic approach, especially with oxidoreductase enzymes due to their known potential for oxidizing recalcitrant pollutants. The ligninolytic enzymes obtained from WRF are relatively non-specific towards organic compounds and they use the free radical mechanism to catalyze the degradation of a wide range of micropollutants (Rodriguez et al., 2004; Wen et al., 2009). The capability of these enzymes was first employed in 1980s to degrade different organic compounds, such as pesticides, dyes, polyaromatic hydrocar-

Table 1

Physical-chemical properties and therapeutic functions of selected pharmaceuticals (Nghiem et al., 2010; Taheran et al., 2016).

\begin{tabular}{|c|c|c|c|c|c|c|c|}
\hline Compound & Acronym & Molecular weight (g/mol) & Molecular formula & Classification & Water solubility $(\mathrm{mg} / \mathrm{L})$ & $\log \mathrm{K}_{\mathrm{ow}}$ & $\mathrm{pKa}$ \\
\hline Acetaminophen & $\mathrm{ACT}$ & 151 & $\mathrm{C}_{8} \mathrm{H}_{9} \mathrm{NO}_{2}$ & Analgesic, antipyretic & 14000 & 0.46 & 9.5 \\
\hline Amitriptyline & AMP & 277.403 & $\mathrm{C}_{20}^{\circ} \mathrm{H}_{23} \mathrm{~N}$ & Antidepressant & 9.71 & 4.92 & 9.4 \\
\hline Atenolol & ATL & 266.336 & $\mathrm{C}_{14} \mathrm{H}_{22} \mathrm{~N}_{2} \mathrm{O}_{3}$ & Antihypertensive agent & 13300 & 0.16 & 9.6 \\
\hline Bezafibrate & $\mathrm{BFB}$ & 361.82 & $\mathrm{C}_{19} \mathrm{H}_{20} \mathrm{ClNO}_{4}$ & Lipid regulator & Slight & 4.25 & 3.44 \\
\hline Caffeine & $\mathrm{CAF}$ & 194.19 & $\mathrm{C}_{8} \mathrm{H}_{10} \mathrm{~N}_{4} \mathrm{O}_{2}$ & Stimulant drug & 21600 & -0.07 & 14 \\
\hline Carbamazepine & $\mathrm{CBZ}$ & 236.27 & $\mathrm{C}_{15} \mathrm{H}_{12} \mathrm{~N}_{2} \mathrm{O}$ & Anti-epileptic & 17.7 & 2.45 & 13.9 \\
\hline Cetirizine & CET & 388.89 & $\mathrm{C}_{21} \mathrm{H}_{25} \mathrm{ClN}_{2} \mathrm{O}_{3}$ & Antihistamine & 101 & 1.70 & $\begin{array}{r}\mathrm{P}_{1}=2.70 \\
\mathrm{P}_{2}=3.57 \\
\mathrm{P}_{3}=7.56\end{array}$ \\
\hline Chlortetracycline & CTC & 478.882 & $\mathrm{C}_{22} \mathrm{H}_{23} \mathrm{ClN}_{2} \mathrm{O}_{8}$ & Antibiotic & 8.6 & -0.68 & $\begin{array}{r}\mathrm{P}_{1}=3.30 \\
\mathrm{P}_{2}=7.55 \\
\mathrm{P}_{3}=9.33\end{array}$ \\
\hline Ciprofloxacine & $\mathrm{CPF}$ & 331.346 & $\mathrm{C}_{17} \mathrm{H}_{18} \mathrm{FN}_{3} \mathrm{O}_{3}$ & Antibiotic & 30000 & 0.28 & 6.09 \\
\hline Citalopram & CTL & 324.392 & $\mathrm{C}_{20} \mathrm{H}_{21} \mathrm{FN}_{2} \mathrm{O}$ & Antidepressant & 5.88 & 3.5 & 9.78 \\
\hline Diazepam & DZP & 284.70 & $\mathrm{C}_{16} \mathrm{H}_{13} \mathrm{ClN}_{2} \mathrm{O}$ & Tranquilizers & 50 & 2.82 & 3.4 \\
\hline Diclofenac & DCF & 296.15 & $\mathrm{C}_{14} \mathrm{H}_{11} \mathrm{C}_{12} \mathrm{NO}_{2}$ & Anti-inflammatory, analgesic & 2.37 & 4.51 & 4.08 \\
\hline Doxycycline & $\mathrm{DC}$ & 444.43 & $\mathrm{C}_{22} \mathrm{H}_{24} \mathrm{~N}_{2} \mathrm{O}_{8}$ & Antibiotic & 630 & 3.5 & $\begin{array}{c}\mathrm{P}_{1}=3.4 \\
\mathrm{P}_{2}=7.7 \\
\mathrm{P}_{3}=9.7\end{array}$ \\
\hline Enrofloxacin & $\mathrm{EFC}$ & 359.4 & $\mathrm{C}_{19} \mathrm{H}_{22} \mathrm{FN}_{3} \mathrm{O}_{3}$ & Antibiotic & 146 & 3.48 & $\begin{array}{c}\mathrm{P}_{1}=5.94 \\
\mathrm{P}_{2}=8.70\end{array}$ \\
\hline Erythromycin & ETM & 733.93 & $\mathrm{C}_{37} \mathrm{H}_{67} \mathrm{NO}_{13}$ & Antibiotic & 2000 & 3.06 & 8.9 \\
\hline Fenofibrate & FEF & 360.831 & $\mathrm{C}_{20} \mathrm{H}_{21} \mathrm{ClO}_{4}$ & Anti-hyperlipidemic & 250 & 5.19 & -4.9 \\
\hline Fenoprofen & FEP & 242 & $\mathrm{C}_{15} \mathrm{H}_{14} \mathrm{O}_{3}$ & Anti-inflammatory & Slight & 3.9 & 4.21 \\
\hline Fluoxetine & FLX & 309.30 & $\mathrm{C}_{17} \mathrm{H}_{18} \mathrm{~F}_{3} \mathrm{NO}$ & Anti-depressants & 50 & 4.05 & 8.7 \\
\hline Gemfibrozil & GFZ & 250.34 & $\mathrm{C}_{15} \mathrm{H}_{22} \mathrm{O}_{3}$ & Lipid regulator & 11 & 4.77 & 4.45 \\
\hline Ibuprofen & IBP & 206.29 & $\mathrm{C}_{13} \mathrm{H}_{18} \mathrm{O}_{2}$ & Anti-inflammatory, analgesic & 21 & 3.97 & 4.47 \\
\hline Indomethacin & IDM & 357.78 & $\mathrm{C}_{19} \mathrm{H}_{16} \mathrm{ClNO}_{4}$ & Anti-inflammatory & 0.937 & 4.23 & 3.8 \\
\hline Ketoprofen & KEP & 254.28 & $\mathrm{C}_{16} \mathrm{H}_{14} \mathrm{O}_{3}$ & Anti-inflammatory, analgesic & 51 & 3.12 & 4.29 \\
\hline Mefenamic acid & MFA & 241.285 & $\mathrm{C}_{15} \mathrm{H}_{15} \mathrm{NO}_{2}$ & Anti-inflammatory & 20 & 5.12 & 3.8 \\
\hline Naproxen & NPX & 230 & $\mathrm{C}_{14} \mathrm{H}_{14} \mathrm{O}_{3}$ & Anti-inflammatory, analgesic & 15.9 & 3.18 & 4.2 \\
\hline Norfloxacin & NOR & 319.331 & $\mathrm{C}_{16} \mathrm{H}_{18} \mathrm{FN}_{3} \mathrm{O}_{3}$ & Antibiotic & 178000 & 0.46 & $\begin{array}{c}\mathrm{P}_{1}=6.34 \\
\mathrm{P}_{2}=8.75\end{array}$ \\
\hline Oseltamivir & OST & 312.40 & $\mathrm{C}_{16} \mathrm{H}_{28} \mathrm{~N}_{2} \mathrm{O}_{4}$ & Antiviral & 1600 & 0.95 & 7.7 \\
\hline Oxytetracycline & OTC & 460.434 & $\mathrm{C}_{22} \mathrm{H}_{24} \mathrm{~N}_{2} \mathrm{O}_{9}$ & Antibiotic & 313 & -0.90 & $\begin{array}{c}\mathrm{P}_{1}=3.3 \\
\mathrm{P}_{2}=7.3 \\
\mathrm{P}_{3}=9.1\end{array}$ \\
\hline Paracetamol & PCT & 151.163 & $\mathrm{C}_{8} \mathrm{H}_{9} \mathrm{NO}_{2}$ & Analgesic, antipyretic & 13000 & 0.34 & 9.5 \\
\hline Propranolol & PPL & 259.34 & $\mathrm{C}_{16} \mathrm{H}_{21} \mathrm{NO}_{2}$ & Beta-blocker & 61.7 & 3.48 & 9.6 \\
\hline Propyphenazone & PPZ & 230.306 & $\mathrm{C}_{14} \mathrm{H}_{18} \mathrm{~N}_{2} \mathrm{O}$ & Anti-pyretic, anti-inflammatory & 2400 & 1.94 & 0.8 \\
\hline Sulfadimethoxine & SDM & 310.33 & $\mathrm{C}_{12} \mathrm{H}_{14} \mathrm{~N}_{4} \mathrm{O}_{4} \mathrm{~S}$ & Antibacterial & 343 & 1.63 & 5.9 \\
\hline Sulfamethazine & $\mathrm{SMZ}$ & 278.33 & $\mathrm{C}_{12} \mathrm{H}_{14} \mathrm{~N}_{4} \mathrm{O}_{2} \mathrm{~S}$ & Antibacterial & 1500 & 0.14 & $\begin{array}{c}P_{1}=2.65 \\
P_{2}=7.65\end{array}$ \\
\hline Sulfamethoxazole & SMX & 253.3 & $\mathrm{C}_{10} \mathrm{H}_{11} \mathrm{~N}_{3} \mathrm{O}_{3} \mathrm{~S}$ & Antibiotic & 610 & 0.89 & $\begin{array}{c}\mathrm{P}_{1}=1.7 \\
\mathrm{P}_{2}=5.6\end{array}$ \\
\hline Sulfamonomethoxine & SMM & 280.302 & $\mathrm{C}_{11} \mathrm{H}_{12} \mathrm{~N}_{4} \mathrm{O}_{3} \mathrm{~S}$ & Antibiotic & 10000 & -0.04 & 5.9 \\
\hline Sulfapyridine & SPY & 249.29 & $\mathrm{C}_{11} \mathrm{H}_{11} \mathrm{~N}_{3} \mathrm{O}_{2} \mathrm{~S}$ & Antibiotic & 268 & 0.35 & 8.43 \\
\hline Sulfathiazole & STZ & 255.319 & $\mathrm{C}_{9} \mathrm{H}_{9} \mathrm{~N}_{3} \mathrm{O}_{2} \mathrm{~S}_{2}$ & Antibacterial & 373 & 0.05 & $\begin{array}{l}P_{1}=2.2 \\
P_{2}=7.24\end{array}$ \\
\hline Sulfonamides sulfanilamide & SAA & 172.20 & $\mathrm{C}_{6} \mathrm{H}_{8} \mathrm{~N}_{2} \mathrm{O}_{2} \mathrm{~S}$ & Antibacterial & 7500 & -0.62 & $\begin{array}{c}\mathrm{P}_{1}=10.43 \\
\mathrm{P}_{2}=11.63\end{array}$ \\
\hline Tetracycline & $\mathrm{TC}$ & 444.435 & $\mathrm{C}_{22} \mathrm{H}_{24} \mathrm{~N}_{2} \mathrm{O}_{8}$ & Antibiotic & 231 & -1.37 & 3.3 \\
\hline Trimethoprim & TMP & 290.32 & $\mathrm{C}_{14} \mathrm{H}_{18} \mathrm{~N}_{4} \mathrm{O}_{3}$ & Antibacterial & 400 & 0.91 & 7.2 \\
\hline
\end{tabular}


bons, etc. (Bumpus et al., 1985). and in 1990s, the researchers showed the applicability of these enzymes for degradation of pharmaceutical compounds (Bauer et al., 1999; Martens et al., 1996).

Many review papers have been recently published to cover the occurrence and fate of micropollutants in the aquatic environment as well as their removal by conventional and advanced treatment processes i.e. adsorption, AOPs and membrane separations (Asif et al., 2017; Grandclément et al., 2017; Taheran et al., 2016). However, there is no comprehensive review to cover the approaches of biocatalytic treatment of pollutants including whole fungal culture, crude/ pure enzyme, immobilized enzyme, and the combination of enzymatic treatment with other technologies. The aim of this work is to review the performance of ligninolytic enzymes for removal of $\mathrm{PhACs}$ from aqueous media. Different types of fungi and their intracellular and extracellular enzymes in free and immobilized forms used to treat micropollutants as well as the effects of operating conditions on removal efficiencies are discussed.

\section{Enzymatic treatment for removal of PhACs}

Enzymes are biologically-made catalysts that mediate biochemical reactions at a rapid rate and can play a crucial role in preventing pollution through cleaner methods for production of substances (Arora and Sharma, 2010). For some commercial processes, such as synthesis of enantiomerically pure intermediates (Carvalho et al., 2015), enzymes have been used and they showed advantages over synthetic catalysts with respect to substrate specificity, working under mild conditions, energy input and posing no toxicity.

Recently, enzymes were employed for transformation of pollutants in different effluents into other less harmful compounds in lab scale reactors (Arora and Sharma, 2010; Christian et al., 2005c; Duran and Esposito, 2000). A biological promising alternative to conventional treatment in WWTPs may be developed based on the use of living cultures or extracted enzymes of fungi such as WRF. These microorganisms have been reported to be able to degrade a wide spectrum of xenobiotics due to the action of extracellular oxidoreductase enzymes, such as lignin peroxidase (LiP), manganese peroxidase (MnP), versatile peroxidase (VP) and laccase (Lac). This consortium of oxidoreductase enzymes is also known as ligninolytic enzymes since they have produced by the fungi for decomposing the lignin-rich biomass into nutrients (Garcia-Ruiz et al., 2014). LiP (EC 1.11.1.14) catalyzes the depolymerization of lignin through the $\mathrm{H}_{2} \mathrm{O}_{2}$-based oxidative process. LiP showed the capability to degrade several recalcitrant aromatic pollutants (Christian et al., 2005c). The molecular mass and optimum temperature and $\mathrm{pH}$ of LiP fall within $37-50 \mathrm{kDa}, 35-55^{\circ} \mathrm{C}$ and $2-5$, respectively (Asgher et al., 2007; Christian et al., 2005b; Hirai et al., 2005). MnP (EC 1.11.1.13) is a heme glycoprotein enzyme that can catalyze the oxidation of organic molecules in the presence of $\mathrm{H}_{2} \mathrm{O}_{2}$ (Wong, 2009). The molecular mass and optimum temperature and $\mathrm{pH}$ of $\mathrm{MnP}$ fall within 32-62.5 kDa, $40-60{ }^{\circ} \mathrm{C}$, and $4-7$ respectively. The different isoforms of $\mathrm{MnP}$ are secreted in nitrogen and carbon-limited media supplemented with VEA and $\mathrm{Mn}^{2+}$ (Baborová et al., 2006; Cheng et al., 2007). VP (EC 1.11.1.16) combines the substrate-specificity of MnP and LiP and is able to oxidize different types of molecular structures such as low- and high-redox-potential dyes, phenolic/non-phenolic compounds as given in Table 1 and hydroquinones (Camarero et al., 1999). Lac (EC 1.10.3.2) is a member of multicopper enzymes family with low-specificity. It can catalyze the oxidation of hydrogen-donating compounds such as phenol, lignin, or acrylamines through the reduction of $\mathrm{O}_{2}$ to $\mathrm{H}_{2} \mathrm{O}$ (Wong, 2009; Yang et al., 2013b). The molecular mass, optimum temperature and $\mathrm{pH}$ of Lac fall within 58-90 kDa, 40-65 ${ }^{\circ} \mathrm{C}$ and 2-10, respectively (Quaratino et al., 2007; Zouari-Mechichi et al., 2006). Among oxidoreductase enzymes, Lac is of great interest since it only needs gaseous oxygen as a co-substrate (Nguyen et al., 2014c). Therefore, low specificity, ability to use atmospheric oxygen as the electron acceptor and good yields make Lac attractive for environmental applications (Lundell et al., 2010; Martínková et al., 2016; Strong and Claus, 2011). In Table 2, the properties of mostly used oxidoreductase enzymes in degradation of PhACs are summarized.

The hydrophobicity of PhACs that can be expressed by $\log \mathrm{K}_{\mathrm{ow}}$ (Table 1), is a key parameter that governs the sorption onto biomass and can enhance the removal of some compounds. For instance, Yang et al. investigated the contribution of biodegradation by extracellular enzymes and biosorption and reported that the removal of hydrophobic compounds ( $\left.\log \mathrm{K}_{\mathrm{ow}}>4\right)$ was highly impacted by both mechanisms. They also found that the biosorption of hydrophobic com-

Table 2

Enzyme properties and some of their application (Asgher et al., 2008; Duran and Esposito, 2000; Husain and Husain, 2007).

\begin{tabular}{|c|c|c|c|c|c|c|}
\hline Enzymes & Acronym & Source & $\begin{array}{l}\text { Molecular weight } \\
(\mathrm{kDa})\end{array}$ & Optimum condition & $\begin{array}{l}\text { Co- } \\
\text { substrate }\end{array}$ & Applications \\
\hline Laccase & Lac & $\begin{array}{l}\text { Funalia trogii } \\
\text { Fomas annosus } \\
\text { Cerrena unicolor } \\
\text { Trametes hispida } \\
\text { Daedalea quercina } \\
\text { Coriolus versicolor } \\
\text { Trametes versicolor } \\
\text { Pycnoporus cinnabarinus }\end{array}$ & $58-90$ & $\begin{array}{l}\text { Temperature: } 40-65^{\circ} \mathrm{C} \\
\text { pH: } 2-10\end{array}$ & $\mathrm{O}_{2}$ & Dyes decoloration and degradation \\
\hline Tyrosinase & Tyros & Agaricus bisporus & $119.5-133$ & $\begin{array}{l}\text { Temperature: } 20-40{ }^{\circ} \mathrm{C} \mathrm{pH}: \\
5-8\end{array}$ & $\mathrm{O}_{2}$ & Phenols and amines degradation \\
\hline Lignin peroxidase & $\mathrm{LiP}$ & $\begin{array}{l}\text { Phanerochaete } \\
\text { chrysosporium }\end{array}$ & $37-50$ & $\begin{array}{l}\text { Temperature: } 35-55^{\circ} \mathrm{C} \\
\text { pH: } 2-5\end{array}$ & $\mathrm{H}_{2} \mathrm{O}_{2}$ & $\begin{array}{l}\text { Phenolic and Aromatic compounds } \\
\text { degradation }\end{array}$ \\
\hline Versatile peroxidase & VP & $\begin{array}{l}\text { Pleurotus eryngii } \\
\text { Bjerkandera adusta }\end{array}$ & $38-45$ & $\begin{array}{l}\text { Temperature: } 15-50{ }^{\circ} \mathrm{C} \mathrm{pH} \text { : } \\
3-5\end{array}$ & $\mathrm{H}_{2} \mathrm{O}_{2}$ & Textile effluent degradation \\
\hline $\begin{array}{l}\text { Manganese } \\
\text { peroxidase }\end{array}$ & $\mathrm{MnP}$ & $\begin{array}{l}\text { Phlebia radiata } \\
\text { Lentinula edodes } \\
\text { Pleurotus ostreatus } \\
\text { Phanerochaete } \\
\text { chrysosporium }\end{array}$ & $32-62.5$ & $\begin{array}{l}\text { Temperature: } 40-60^{\circ} \mathrm{C} \mathrm{pH} \text { : } \\
4-7\end{array}$ & $\mathrm{H}_{2} \mathrm{O}_{2}$ & Phenols, lignins and dyes degradation \\
\hline
\end{tabular}


pounds facilitated their biodegradation (Yang et al., 2013c). On the other hand, the role of biosorption in the removal of the hydrophilic compounds $\left(\log \mathrm{K}_{\mathrm{ow}}<3\right)$ is limited so that for some compounds, the effect of biosorption was reported to be negligible compared to the biodegradation. Since whole-cell fungal treatment involves extracellular, intracellular, and mycelium-bound enzymes, there are significant differences in treatment by whole-cell WRF and extracted enzymes. The complete removal of some compounds in whole-cell fungal reactors indicates the important role of intracellular and mycelium-bound enzymes and their synergistic effect with extracellular enzymes (Nguyen et al., 2014a, 2013, 2016b).

Laccase, as illustrated in Fig. 1A, has four 4 copper atoms divided into three types (1,2 and 3$)$ at the catalytic center of each monomer. The type 1 atom (T1) imparts the color of the enzyme and catalyzes the oxidation of the substrate. Afterwards, the donated electron from the substrate is internally transferred from $\mathrm{T} 1$ to the $\mathrm{T} 2$ and T3 copper sites where the reduction of oxygen to water takes place (Fernández-Fernández et al., 2013; Senthivelan et al., 2016). The oxidation in $\mathrm{T} 1$ is a one-electron reaction that generates a radical, and the reduction in T2 and T3 sites is a four-electron reaction that produces two molecules of water. The initial free radical is fairly unstable and may be transformed to a quinone by spontaneous disproportionation or in a second enzyme-catalyzed step. For phenolic polymers, such as humic acids, further non-enzymatic radical reactions are also possible that may result in their partial degradation (Strong and Claus, 2011).

LiP has a high redox potential and therefore it can oxidize the compounds that are not oxidized by other enzymes. It can attack both phenolic and non-phenolic structures and lead to hydroxylation, carbon-carbon cleavage, phenolic oxidation, methylation, aromatic ring fission, demethoxylation and dimerization reactions. LiP follows a well-known peroxidase catalytic mechanism in which native enzyme is oxidized by hydrogen peroxide and forms LiP-I with two electron deficiencies. LiP-I oxidizes the target compound and reduces to one electron deficient LiP-II. When LiP-II oxidizes another target molecule, it returns to the native form of LiP. Due to low mobility and accessibility of enzyme active sites for target compounds, the involvement of low molecular weight redox mediator plays an important role. Veratryl alcohol (VEA) is naturally produced by WRF and serves as a mediator to facilitate oxidation of substrates. VEA can be oxidized by $\mathrm{LiP}$ to a cationic radical species $\left(\mathrm{VEA}^{\bullet+}\right)$ which is responsible for the oxidation of target compounds (Gold et al., 1989; Kersten, 1990).

$\mathrm{MnP}$, as illustrated in Fig. $1 \mathrm{~B}$, oxidizes $\mathrm{Mn}^{2+}$ to $\mathrm{Mn}^{3+}$ that can be stabilized by chelators, such as organic acids and acts as a redox mediator to attack organic compounds and oxidize them through abstraction of one hydrogen and one electron. Similar to LiP, MnP first reacts with hydrogen peroxide and form MnP-I with two electron deficiency. MnP-I oxidizes the target compounds and is transformed to $\mathrm{MnP}-\mathrm{II}$ which is slowly reduced to native $\mathrm{MnP}$ and needs $\mathrm{Mn}^{2+}$ to complete the catalytic cycle (Christian et al., 2005a).

A single fungal species is not able to produce all four extracellular enzymes and the combination of ligninolytic enzymes varies from one WRF species to another. Even the secretion profile of enzymes varies among WRF species. Furthermore, the nutrient composition e.g. carbon and nitrogen and conditions of growth media e.g. temperature and $\mathrm{pH}$ can influence the secretion of enzymes (Yang et al., 2013c). Apart from the mentioned enzymes, an intracellular enzyme system in WRF i.e. cytochrome P450 (CYP450) was found to play a significant role in the degradation of some pollutants (Golan-Rozen et al., 2011). Therefore, employing these fungi for removal of PhACs can be divided into three categories of: (i) using whole-cell culture; (ii) using crude culture extract or pure enzyme; and (iii) using immobilized enzymes (Marco-Urrea et al., 2009; Taheran et al., 2017a; Zhang and Geißen, 2010).

\subsection{Whole-cell fungal culture}

Among fungal species, WRF is able to efficiently remove a wide range of organic compounds that are resistant to bacterial degradation (Hata et al., 2010b). This capability comes from the action of the intracellular system i.e. CYP450 and extracellular ligninolytic enzymes i.e. LiP, MnP and Lac (Hata et al., 2010b; Nguyen et al., 2013). Because of the combined effect of intracellular/extracellular enzymes and sorption of PhACs on the biomass, whole-cell fungal treatment can remove a wider spectrum of PhACs, such as antibiotics, anti-inflammatories and antiepileptics compared to the case of using a single enzyme (Hata et al., 2010a; Marco-Urrea et al., 2009; Tran et al., 2010). Several properties of WRF make them attractive for application in removal of PhACs, such as: (1) non-specificity of their produced enzyme which enables the degradation of a wide range of micropollutants; (2) the fast colonization through hyphal growth which enables WRF to access pollutants; (3) production and secretion of enzymes to degrade compounds with low water solubility; and (4) the ability to degrade compounds in nutrient deficient media over a wide $\mathrm{pH}$ range of $3-9$. It is noteworthy that the degradation of persistent pollutant by WRF is a co-metabolic process which means it happens in the presence of a readily degradable substrate (Pointing, 2001; Rouches et al., 2016). The necessity of co-substrate addition, typically glucose, is a drawback which increases the cost but simultaneously increases the degradation efficiency (González et al., 2010). An illustration of pollutant removal with the fungal cell, as discussed earlier, is presented in Fig. 2. Accordingly, the target pollutants can be
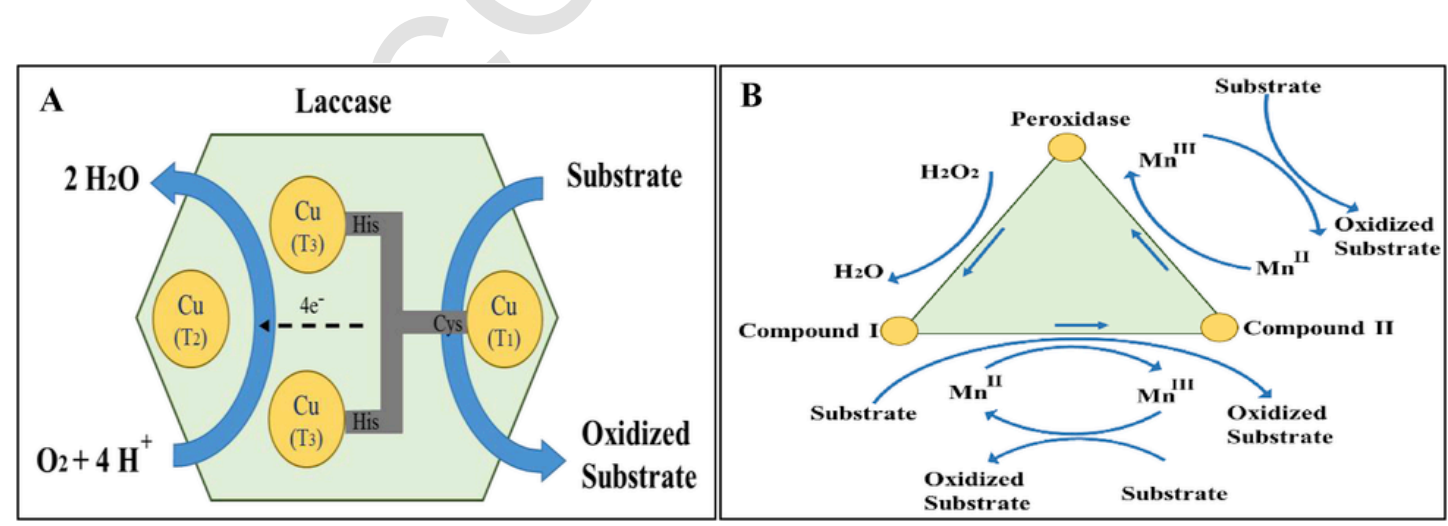

Fig. 1. Mechanism of oxidation of compounds: (a) by the laccase enzyme and; (b) by peroxidase enzyme. 


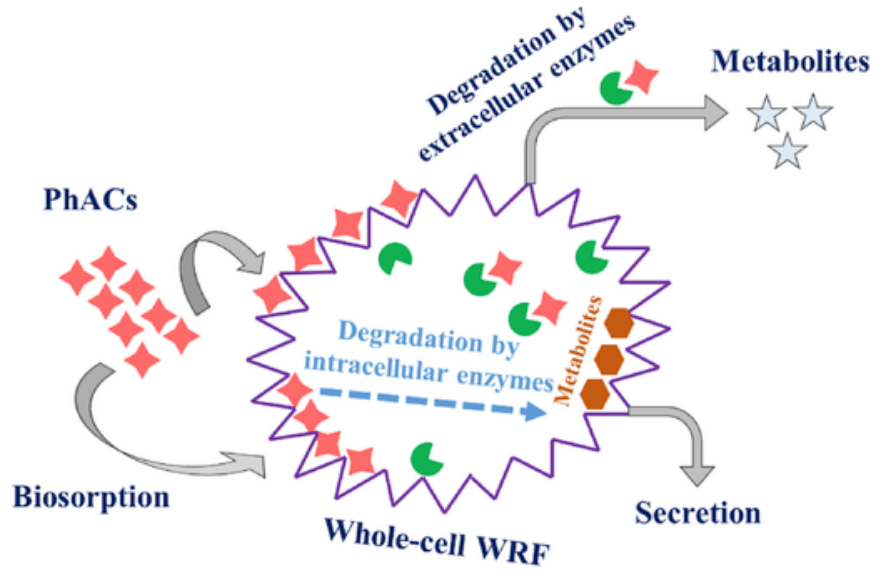

Fig. 2. A schematic illustration of pollutant removal by white-rot fungi.

adsorbed on the surface of fungi or into the cell and later degraded by extracellular and intracellular enzymes.

The removal efficiency of pollutants by WRF can be affected by sorption, which is significant for compounds with a high octanol/water $\left(\mathrm{K}_{\mathrm{ow}}\right)$ partition coefficient (Yang et al., 2013c). For example, Guo et al. studied the degradation of SMX by the fungus, Phanerochaete chrysosporium (PC) in whole fungal culture and also with extracted crude Lac. In the case of whole fungal culture with Lac activity of around $1500 \mathrm{U} / \mathrm{L}$, they reported 53\% degradation efficiency of SMX after $24 \mathrm{~h}$ when initial SMX concentration was $10 \mathrm{mg} / \mathrm{L}$ and less than $3 \%$ removal efficiency due to biosorption. While in the case of using crude enzyme at $6076 \mathrm{U} / \mathrm{L}$ of Lac activity, they observed SMX degradation of $42 \%$, in $24 \mathrm{~h}$ (Guo et al., 2014). It is indicated that the sorption of pollutants into the cell and the action of the intracellular enzyme increased the degradation efficiency. However, systematic investigation on the contribution of biosorption and biodegradation during fungal removal of PhACs will be useful for designing an efficient and stable fungal reactor for removal of micropollutants (Yang et al., 2013c). For example, Yang et al. studied the removal of DCF by whole-cell of the WRF Trametes versicolor (TV). DCF showed high initial sorption $(44 \pm 13 \%)$ and high removal efficiency $(>90 \%)$. Lucas et al. reported that the contribution of the sorption process to overall removal depends on the fungal strain and the interactions between PhACs and the components of fungal surface. Among the six fungal strains, they related the minimum and maximum removal by sorption to Stropharia rugosoannulata (4\%) and Ganoderma lucidum (26\%) (Lucas et al., 2018). According to Table 1, the relatively hydrophobic nature of DCF $\left(\mathrm{K}_{\mathrm{ow}}=4.51\right)$ plays an important role in DCF sorption to fungal cells. Comparison between the whole fungal culture and extracted enzyme (30\% degradation efficiency) indicated that a pathway independent of extracellular Lac was responsible for removal of DCF (Yang et al., 2013c).

The removal mechanisms involved in treatment with WRF whole-culture can be divided into three steps including sorption onto biomass, biodegradation by extracellular enzymes e.g. Lac and degradation by intracellular or mycelium-bound enzymes (Fig. 2). In addition to biosorption, there are other factors, including pollutant structure, fungal species, enzyme systems, culture medium, $\mathrm{pH}$, temperature and enhancing methods e.g. the presence of mediators that affects the removal performance of a WRF (Rodarte-Morales et al., 2011; Tran et al., 2010). For instance, TV, which seems to have a good potential for the degradation of micropollutants, secretes three types of ligninolytic enzymes i.e. Lac, LiP and MnP among which Lac is the predominant one in some strains (Cruz-Morató et al., 2013; Nguyen et al., 2014d). This fungi showed better performance in aqueous media than solid matrices that can be due to the better mass transport in liquid media. As an instance, DCF, IBP, and NPX were almost completely removed in liquid media of TV (Marco-Urrea et al., 2009; Tran et al., 2010) while their removal efficiencies in solid media of TV were 64\%, 75\% and 47\%, respectively (Rodríguez-Rodríguez et al., 2011). Furthermore, the degradation ability of different species of WRFs and even different strains of one species is not similar. Similar behavior is not expected for one kind of crude enzyme extracted from different fungi. For example, LiP from Phanerochaete sordida (PS) showed higher efficiency for removal of some pollutants compared to LiP from PC (Wang et al., 2012). Fungi other than WRF also showed capability to degrade PhACs. For example, Pestalotiopsis guepini strain P-8 showed $67.7 \%$ and $68.9 \%$ removal efficiency for CPF and NOR after 18 days with initial concentration of $300 \mu \mathrm{M}$ and $313 \mu \mathrm{M}$, respectively (Parshikov et al., 2001). In another study, three strains of Mucor ramannianus grown on the malt/sucrose medium were used for biodegradation of antimalarial drug artemisinin. These strains transformed the artemisinin into $7 \beta-6 \beta$-hydroxyartemisinin at $51 \%$ yield and hydroxyartemisinin at $88 \%$ yield (Parshikov et al., 2005). Recent studies showed that the hydroxylated derivatives of this compound possess higher anti-malarial activity and water solubility (Zhan et al., 2017). Also, the saprobic fungus, Mucor ramannianus, demonstrated $89.1 \%$ removal efficiency for CPF (initial concentration of $100 \mathrm{mg} / \mathrm{L}$ ) after 14 days (Parshikov et al., 1999).

\subsubsection{Role of intracellular and extracellular enzymes}

The role of mycelium-related enzymes and intracellular systems especially CYP450 in biodegradation of PhACs have been intensely studied for compounds, such as CTL, SMX, DCF, IBP, CBZ, SMZ, NPX and KEP (García-Galán et al., 2011; Marco-Urrea et al., 2010c; Nguyen et al., 2014d; Rodarte-Morales et al., 2011). For example, Hata et al. demonstrated that the WRF PS YK-624 can degrade and remove the acute lethal toxicity of MFA and DCF towards Thamnocephalus platyurus (the freshwater crustacean) within 6 days. They suggested that catalytic hydroxylation by CYP450 is responsible for detoxification of MFA and DCF (Hata et al., 2010a). Also, Golan-Rozen et al. studied three strains of Pleurotus ostreatus (PO) and noticed the significance of CYP450 and MnP in degradation of CBZ (Golan-Rozen et al., 2011). Their results implied that when both $\mathrm{MnP}$ and CYP450 systems were involved, $99 \%$ of the CBZ was transformed to 10,11 Epoxy-CBZ. This end-product is an active pharmaceutical compound and its presence in waterways is not desirable (Buchicchio et al., 2016). However, when both MnP and CYP450 were inactivated, less than $30 \% \mathrm{CBZ}$ removal was achieved (Golan-Rozen et al., 2011). In a related study, Marco-Urrea et al. studied the degradation of IBP and CBZ at $10 \mathrm{mg} / \mathrm{L}$ by four WRF i.e. TV, Irpex lacteus, Ganoderma lucidum and PC within 7 days. Accordingly, all strains except PC almost completely degraded IBP. The in vitro tests showed that although intracellular systems play a major role in degradation of IBP, CYP450 did not affect this process. In contrast, inhibition of CYP450 reduced the degradation efficiency of CBZ by more than 57\% (Marco-Urrea et al., 2009). Later, they studied the degradation of NPX at two different concentrations $(10 \mathrm{mg}$ / $\mathrm{L}$ and $55 \mu \mathrm{g} / \mathrm{L})$ in a liquid medium of TV and achieved $>99 \%$ and $95 \%$ of degradation after 6 and $5 \mathrm{~h}$, respectively. In this case, they observed that Lac and the CYP450 system was responsible for degradation of NPX (Marco-Urrea et al., 2010a). Similarly, Prieto et al. reported the inhibition of NOR and CPF degradation by addition of CYP450 inhibitor to TV grown on malt extract liquid medium which indicated the important role of CYP450 in degradation of the two antibiotics (Prieto et al., 2011). However, Rodriguez-Rodriguez et al. studied the removal of 
NPX in sewage sludge samples with TV and observed that NPX values did not necessarily correlate with CYP450 or Lac amounts though in most cases, over 30\% degradation happened (Rodríguez-Rodríguez et al., 2010). They also found that addition of CYP450 inhibitor to the TV culture partially suppresses the degradation STZ but has no effect was on degradation of SPY (Rodríguez-Rodríguez et al., 2012). To sum up, both intra-cellular and extracellular enzymes play key roles in the degradation of PhACs, but depending on the compounds, they act differently. The intracellular enzymes may advance the first step of $\mathrm{PhACs}$ oxidation, while, extracellular enzymes do not intervene in the first step of degradation.

\subsubsection{Bioreactors}

Developing an effective setup to facilitate preparation, handling and implementation of reactor system is essential for wide application of bioremediation with WRF. Different reactor configurations have been studied for the treatment of PhACs with enzymes (Rodarte-Morales et al., 2012a, b) and the data on degradation efficiencies of different systems are listed in Table 3. The performance of WRF for removal of PhACs has been often studied on synthetic wastewater containing high concentrations of contaminants (up to several $\mathrm{mg} / \mathrm{L}$ ) under sterile conditions to avoid contamination with bacterial strains (Grandclément et al., 2017). Contamination with bacteria in the fungal bioreactor has adverse effects on removal efficiency of PhACs since they compete with fungi for substrate, disrupt the growth of fungi and damage the mycelium (Espinosa-Ortiz et al., 2016). Hence, it is essential to develop methods for an uninterrupted fungal growth. Some possible strategies to avoid contamination with bacterial are reducing reaction $\mathrm{pH}$ to acidic range, immobilization of fungi, limiting nitrogen in feed, using disinfecting agents and pretreatment of wastewater (Mir-Tutusaus et al., 2016; Van Leeuwen et al., 2003). In few cases, fungal bioreactors were operated under non-sterile conditions for a short period of time and above strategies were investigated (Cruz-Morató et al., 2013; Jelic et al., 2012). For example $\mathrm{Li}$ et al. used a continuous bioreactor packed with a mixture of WRF mycelia pellets under non-sterile condition for 28 days for removal of NPX and CBZ at $1.0 \mathrm{mg} / \mathrm{L}$. They observed $60-80 \%$ removal efficiency of CBZ and complete removal of NPX in the beginning, but the removal efficiencies dropped to less than $20 \%$ by the 14 th day due to the contamination. Addition of sodium hypochlorite into the influent tank increased the removal efficiency to initial level for NPX by inhibiting contamination, but it did not work for CBZ (Li et al., 2015). Cruz-Morato et al. monitored the degradation of $10 \mathrm{PhACs}$ in urban wastewater in a non-sterile batch fluidized bed bioreactor inoculated with TV culture at fixed $\mathrm{pH}$ level of 4.5. According to their observations, TV can remain active in the presence of bacteria and contaminants and addition of nutrients such as nitrogen and glucose can maintain a significant biological activity. They reported that in 8 days, 7 out of the $10 \mathrm{PhACs}$, such as IBP, ACT and KEP were removed completely, 2 of them were removed partially and only one of them (CBZ) showed higher concentration due to deconjugation of compound intermediates (Cruz-Morató et al., 2013). Also, they compared the operation in sterile and non-sterile modes for treatment of hospital wastewater containing more than $8 \mathrm{mg} / \mathrm{L}$ of PhACs and observed $83.2 \%$ and $53.3 \%$ degradation efficiency, respectively. They also employed Microtox test to demonstrate that both treatment can reduce the toxicity of wastewater (Cruz-Morató et al., 2014). In another study, Yang et al. compared the removal of DCF in a sterile TV fungal reactor operated in batch mode (three months of reaction time) and continuous mode (two days of retention time). They observed complete removal in batch mode and 55\% removal efficiency in continuous mode (Yang et al., 2013a). This huge difference can be due to the shorter contact time and loss of enzyme in the continuous reactor.

Despite the lack of Lac and VP, the removal performance of PC towards pharmaceuticals has also been studied (Hatakka, 1994). For instance, Zhang and Geißen grew PC on polyether foam under non-sterile conditions in a plate bioreactor to remove CBZ in continuous mode for 100 days. They found that the supply of nutrients is essential for effective elimination of CBZ. They achieved around $80 \%$ removal efficiency with synthetic wastewater and around $60 \%$ with the real effluent (Zhang and Geißen, 2012). Also, Rodarte-Morales et al. studied the degradation of DCF and IBP and NPX with PC in a fed-batch reactor with continuous air supply over 30 days. They observed the complete removal of IBP and DCF after $23 \mathrm{~h}$ in aerated reactors. These observations implied that the oxidative capacity of $\mathrm{PC}$ for the anti-inflammatory $\mathrm{PhACs}$ is not limited to an oxygen environment since the fungal reactor could remove them under aerated conditions (Rodarte-Morales et al., 2012a). In a related study, they found that working in a continuous reactor with aged PC culture (more than 20 days) is in favor of removal of CBZ $(>90 \%)$. But compared to the fed-batch reactor with fresh $\mathrm{PC}$ culture, it reduced the removal efficiency for anti-inflammatory drugs i.e. DCF, IBP, and NPX from $>99 \%$ to less than $50 \%$ (Rodarte-Morales et al., 2012c). It can be due to wash-out of the enzyme in a continuous system and also the fact that the aged culture of fungi had less enzyme production compared to fresh culture.

As mentioned earlier, most of the researchers worked with synthetic wastewater or spiked wastewater with high concentrations (up to $20 \mathrm{mg} / \mathrm{L}$ ) of PhACs and the results cannot be satisfactorily extrapolated to environmentally relevant concentrations (ng/L to $\mu \mathrm{g} / \mathrm{L}$ ). For example, Jelic et al. performed the aerobic degradation of CBZ in Erlenmeyer flask by TV. At $9 \mathrm{mg} / \mathrm{L}$ of CBZ, they observed $94 \%$ removal after 6 days, while at initial CBZ concentration of $50 \mu \mathrm{g} / \mathrm{L}$, they observed only $61 \%$ removal after 7 days. Also, they performed degradation of $\mathrm{CBZ}$ in an air pulsed fluidized bioreactor in batch and continuous modes and observed $96 \%$ and 54\% degradation efficiency, respectively (Jelic et al., 2012). Comparing the performance of fungi in batch and continuous tests, two inherent restrictions were reported as the main reasons for lowering the removal efficiency from batch to continuous mode i.e. washout of extracellular enzymes with effluent and destabilization of fungal activity by bacteria (Gao et al., 2008; Hai et al., 2009). However, Ferrando-Clement et al. reported that the degradation efficiency of TV towards CPF in hospital wastewater is higher in non-sterile compared to sterile conditions. They attributed it to the synergistic degrading contribution by fungi and fecal bacterial (Ferrando-Climent et al., 2015).

To sum up, it is still required to work on real wastewater containing environmentally-relevant concentrations of different contaminants $(\mathrm{ng} / \mathrm{L}$ to $\mu \mathrm{g} / \mathrm{L})$ under non-sterile conditions in continuous mode to investigate the performance and feasibility of fungal reactors for the treatment of contaminated water and wastewater.

\subsection{Metabolite and toxicity assays}

Degradation of PhACs by enzymes take place through different pathways and several intermediates and end-products are generated during the reaction. In most of the studies, researchers predominantly focused on the disappearance of the parent compounds rather than degradation pathways and toxicity of the TPs (Becker et al., 2016; Rahmani et al., 2015; Sutar and Rathod, 2016). However, the properties of TPs are of high importance for releasing into the environment. In this section, the degradation pathways of some PhACs are dis- 
Table 3

Removal (\%) of PhACs by different species of white rot fungi using different operating conditions.

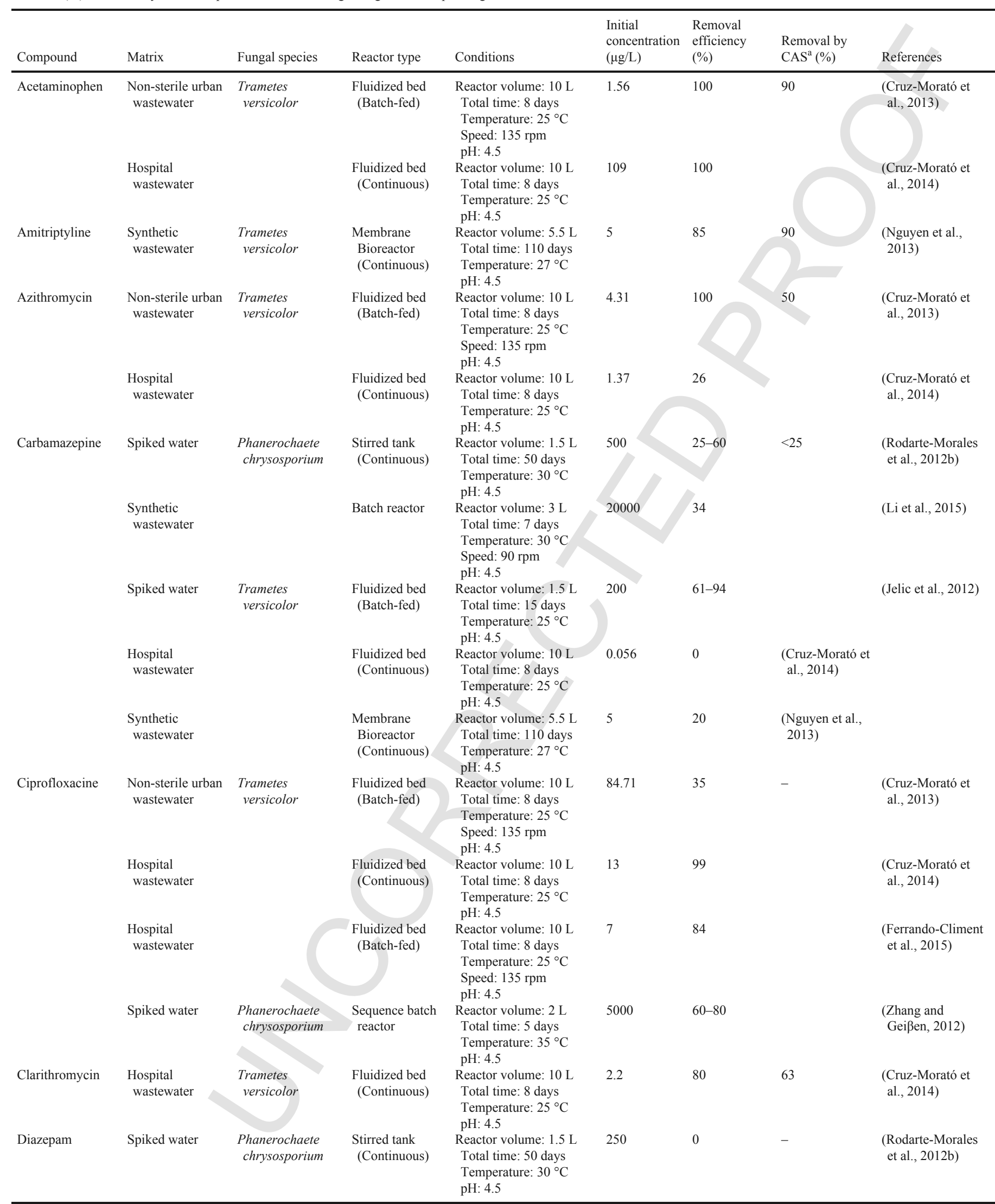


Table 3 (Continued)

\begin{tabular}{|c|c|c|c|c|c|c|c|c|}
\hline Compound & Matrix & Fungal species & Reactor type & Conditions & $\begin{array}{l}\text { Initial } \\
\text { concentration } \\
(\mu \mathrm{g} / \mathrm{L})\end{array}$ & $\begin{array}{l}\text { Removal } \\
\text { efficiency } \\
(\%)\end{array}$ & $\begin{array}{l}\text { Removal by } \\
\operatorname{CAS}^{\mathrm{a}}(\%)\end{array}$ & References \\
\hline \multirow[t]{5}{*}{ Diclofenac } & Spiked water & $\begin{array}{l}\text { Phanerochaete } \\
\text { chrysosporium }\end{array}$ & $\begin{array}{l}\text { Stirred tank } \\
\text { (Batch-fed) }\end{array}$ & $\begin{array}{l}\text { Reactor volume: } 2 \mathrm{~L} \\
\text { Total time: } 30 \text { days } \\
\text { Temperature: } 30^{\circ} \mathrm{C} \\
\text { Speed: } 200 \mathrm{rpm} \\
\text { pH: } 4.5\end{array}$ & 0.8 & $>99$ & 50 & $\begin{array}{l}\text { (Rodarte-Morales } \\
\text { et al., 2012a) }\end{array}$ \\
\hline & Spiked water & & $\begin{array}{l}\text { Stirred tank } \\
\text { (Continuous) }\end{array}$ & $\begin{array}{l}\text { Reactor volume: } 1.5 \mathrm{~L} \\
\text { Total time: } 50 \text { days } \\
\text { Temperature: } 30^{\circ} \mathrm{C} \\
\text { pH: } 4.5\end{array}$ & 1000 & 92 & & $\begin{array}{l}\text { (Rodarte-Morales } \\
\text { et al., 2012b) }\end{array}$ \\
\hline & $\begin{array}{l}\text { Hospital } \\
\text { wastewater }\end{array}$ & $\begin{array}{l}\text { Trametes } \\
\text { versicolor }\end{array}$ & $\begin{array}{l}\text { Fluidized bed } \\
\text { (Continuous) }\end{array}$ & $\begin{array}{l}\text { Reactor volume: } 10 \mathrm{~L} \\
\text { Total time: } 8 \text { days } \\
\text { Temperature: } 25^{\circ} \mathrm{C} \\
\text { pH: } 4.5\end{array}$ & 0.477 & 100 & & $\begin{array}{l}\text { (Cruz-Morató et } \\
\text { al., 2014) }\end{array}$ \\
\hline & $\begin{array}{l}\text { Synthetic } \\
\text { wastewater }\end{array}$ & & $\begin{array}{l}\text { Membrane } \\
\text { Bioreactor } \\
\text { (Continuous) }\end{array}$ & $\begin{array}{l}\text { Reactor volume: } 5.5 \mathrm{~L} \\
\text { Total time: } 90 \text { days } \\
\text { Temperature: } 27^{\circ} \mathrm{C} \\
\text { pH: } 5.4\end{array}$ & 345 & 55 & $\begin{array}{l}\text { (Yang et al., } \\
\text { 2013a) }\end{array}$ & \\
\hline & $\begin{array}{l}\text { Synthetic } \\
\text { wastewater }\end{array}$ & & $\begin{array}{l}\text { Membrane } \\
\text { Bioreactor } \\
\text { (Continuous) }\end{array}$ & $\begin{array}{l}\text { Reactor volume: } 5.5 \mathrm{~L} \\
\text { Total time: } 110 \text { days } \\
\text { Temperature: } 27^{\circ} \mathrm{C} \\
\text { pH: } 4.5\end{array}$ & 5 & 50 & $\begin{array}{l}\text { (Nguyen et al., } \\
\text { 2013) }\end{array}$ & \\
\hline Gemfibrozil & $\begin{array}{l}\text { Synthetic } \\
\text { wastewater }\end{array}$ & $\begin{array}{l}\text { Trametes } \\
\text { versicolor }\end{array}$ & $\begin{array}{l}\text { Membrane } \\
\text { Bioreactor } \\
\text { (Continuous) }\end{array}$ & $\begin{array}{l}\text { Reactor volume: } 5.5 \mathrm{~L} \\
\text { Total time: } 110 \text { days } \\
\text { Temperature: } 27^{\circ} \mathrm{C} \\
\text { pH: } 4.5\end{array}$ & 5 & 95 & - & $\begin{array}{l}\text { (Nguyen et al., } \\
\text { 2013) }\end{array}$ \\
\hline \multirow[t]{6}{*}{ Ibuprofen } & Spiked water & $\begin{array}{l}\text { Phanerochaete } \\
\text { chrysosporium }\end{array}$ & $\begin{array}{l}\text { Stirred tank } \\
\text { (Batch-fed) }\end{array}$ & $\begin{array}{l}\text { Reactor volume: } 2 \mathrm{~L} \\
\text { Total time: } 30 \text { days } \\
\text { Temperature: } 30{ }^{\circ} \mathrm{C} \\
\text { Speed: } 200 \mathrm{rpm} \\
\text { pH: } 4.5\end{array}$ & 0.9 & $75-90$ & 90 & $\begin{array}{l}\text { (Rodarte-Morales } \\
\text { et al., 2012a) }\end{array}$ \\
\hline & Spiked water & & $\begin{array}{l}\text { Stirred tank } \\
\text { (Continuous) }\end{array}$ & $\begin{array}{l}\text { Reactor volume: } 1.5 \mathrm{~L} \\
\text { Total time: } 50 \text { days } \\
\text { Temperature: } 30^{\circ} \mathrm{C} \\
\text { pH: } 4.5\end{array}$ & 1000 & 95 & & $\begin{array}{l}\text { (Rodarte-Morales } \\
\text { et al., 2012b) }\end{array}$ \\
\hline & $\begin{array}{l}\text { Non-sterile urban } \\
\text { wastewater }\end{array}$ & $\begin{array}{l}\text { Trametes } \\
\text { versicolor }\end{array}$ & $\begin{array}{l}\text { Fluidized bed } \\
\text { (Batch-fed) }\end{array}$ & $\begin{array}{l}\text { Reactor volume: } 10 \mathrm{~L} \\
\text { Total time: } 8 \text { days } \\
\text { Temperature: } 25^{\circ} \mathrm{C} \\
\text { Speed: } 135 \mathrm{rpm} \\
\text { pH: } 4.5\end{array}$ & 2.34 & 100 & & $\begin{array}{l}\text { (Cruz-Morató et } \\
\text { al., 2013) }\end{array}$ \\
\hline & $\begin{array}{l}\text { Hospital } \\
\text { wastewater }\end{array}$ & & $\begin{array}{l}\text { Fluidized bed } \\
\text { (Continuous) }\end{array}$ & $\begin{array}{l}\text { Reactor volume: } 10 \mathrm{~L} \\
\text { Total time: } 8 \text { days } \\
\text { Temperature: } 25^{\circ} \mathrm{C} \\
\text { pH: } 4.5\end{array}$ & 35.5 & 100 & $\begin{array}{l}\text { (Cruz-Morató et } \\
\text { al., 2014) }\end{array}$ & \\
\hline & $\begin{array}{l}\text { Synthetic } \\
\text { wastewater }\end{array}$ & & $\begin{array}{l}\text { Membrane } \\
\text { Bioreactor } \\
\text { (Continuous) }\end{array}$ & $\begin{array}{l}\text { Reactor volume: } 5.5 \mathrm{~L} \\
\text { Total time: } 110 \text { days } \\
\text { Temperature: } 27^{\circ} \mathrm{C} \\
\text { pH: } 4.5\end{array}$ & 5 & 95 & $\begin{array}{l}\text { (Nguyen et al., } \\
\text { 2013) }\end{array}$ & \\
\hline & $\begin{array}{l}\text { Hospital } \\
\text { wastewater }\end{array}$ & & $\begin{array}{r}\text { Fluidized bed } \\
\text { (Continuous) }\end{array}$ & $\begin{array}{l}\text { Reactor volume: } 1.5 \mathrm{~L} \\
\text { Total time: } 5 \text { days } \\
\text { Temperature: } 25^{\circ} \mathrm{C} \\
\text { pH: } 4.5\end{array}$ & 20000 & 90 & $\begin{array}{l}\text { (Mir-Tutusaus } \\
\text { et al., 2016) }\end{array}$ & \\
\hline \multirow[t]{4}{*}{ Ketoprofen } & $\begin{array}{l}\text { Non-sterile urban } \\
\text { wastewater }\end{array}$ & $\begin{array}{l}\text { Trametes } \\
\text { versicolor }\end{array}$ & $\begin{array}{l}\text { Fluidized bed } \\
\text { (Batch-fed) }\end{array}$ & $\begin{array}{l}\text { Reactor volume: } 10 \mathrm{~L} \\
\text { Total time: } 8 \text { days } \\
\text { Temperature: } 25^{\circ} \mathrm{C} \\
\text { Speed: } 135 \mathrm{rpm} \\
\text { pH: } 4.5\end{array}$ & 0.08 & 100 & 50 & $\begin{array}{l}\text { (Cruz-Morató et } \\
\text { al., 2013) }\end{array}$ \\
\hline & $\begin{array}{l}\text { Hospital } \\
\text { wastewater }\end{array}$ & & $\begin{array}{l}\text { Fluidized bed } \\
\text { (Continuous) }\end{array}$ & $\begin{array}{l}\text { Reactor volume: } 10 \mathrm{~L} \\
\text { Total time: } 8 \text { days } \\
\text { Temperature: } 25^{\circ} \mathrm{C} \\
\text { pH: } 4.5\end{array}$ & 2.17 & 95 & & $\begin{array}{l}\text { (Cruz-Morató et } \\
\text { al., 2014) }\end{array}$ \\
\hline & $\begin{array}{l}\text { Synthetic } \\
\text { wastewater }\end{array}$ & & $\begin{array}{l}\text { Membrane } \\
\text { Bioreactor } \\
\text { (Continuous) }\end{array}$ & $\begin{array}{l}\text { Reactor volume: } 5.5 \mathrm{~L} \\
\text { Total time: } 110 \text { days } \\
\text { Temperature: } 27^{\circ} \mathrm{C} \\
\text { pH: } 4.5\end{array}$ & 5 & 90 & & $\begin{array}{l}\text { (Nguyen et al., } \\
2013 \text { ) }\end{array}$ \\
\hline & $\begin{array}{l}\text { Hospital } \\
\text { wastewater }\end{array}$ & & $\begin{array}{l}\text { Fluidized bed } \\
\text { (Continuous) }\end{array}$ & $\begin{array}{l}\text { Reactor volume: } 1.5 \mathrm{~L} \\
\text { Total time: } 5 \text { days } \\
\text { Temperature: } 25^{\circ} \mathrm{C} \\
\text { pH: } 4.5\end{array}$ & 20000 & 70 & & $\begin{array}{l}\text { (Mir-Tutusaus et } \\
\text { al., 2016) }\end{array}$ \\
\hline
\end{tabular}


Table 3 (Continued)

\begin{tabular}{|c|c|c|c|c|c|c|c|c|}
\hline Compound & Matrix & Fungal species & Reactor type & Conditions & $\begin{array}{l}\text { Initial } \\
\text { concentration } \\
(\mu \mathrm{g} / \mathrm{L})\end{array}$ & $\begin{array}{l}\text { Removal } \\
\text { efficiency } \\
(\%)\end{array}$ & $\begin{array}{l}\text { Removal by } \\
\operatorname{CAS}^{\mathrm{a}}(\%)\end{array}$ & References \\
\hline \multirow[t]{2}{*}{ Metronidazole } & $\begin{array}{l}\text { Hospital } \\
\text { wastewater }\end{array}$ & $\begin{array}{l}\text { Trametes } \\
\text { versicolor }\end{array}$ & $\begin{array}{l}\text { Fluidized bed } \\
\text { (Continuous) }\end{array}$ & $\begin{array}{l}\text { Reactor volume: } 10 \mathrm{~L} \\
\text { Total time: } 8 \text { days } \\
\text { Temperature: } 25^{\circ} \mathrm{C} \\
\text { pH: } 4.5\end{array}$ & 0.912 & 85 & 38.7 & $\begin{array}{l}\text { (Cruz-Morató et } \\
\text { al., 2014) }\end{array}$ \\
\hline & $\begin{array}{l}\text { Synthetic } \\
\text { wastewater }\end{array}$ & & $\begin{array}{l}\text { Membrane } \\
\text { Bioreactor } \\
\text { (Continuous) }\end{array}$ & $\begin{array}{l}\text { Reactor volume: } 5.5 \mathrm{~L} \\
\text { Total time: } 110 \text { days } \\
\text { Temperature: } 27^{\circ} \mathrm{C} \\
\text { pH: } 4.5\end{array}$ & 5 & 40 & & $\begin{array}{l}\text { (Nguyen et al., } \\
2013 \text { ) }\end{array}$ \\
\hline \multirow[t]{4}{*}{ Naproxen } & Spiked water & $\begin{array}{l}\text { Phanerochaete } \\
\text { chrysosporium }\end{array}$ & $\begin{array}{l}\text { Stirred tank } \\
\text { (Batch-fed) }\end{array}$ & $\begin{array}{l}\text { Reactor volume: } 2 \mathrm{~L} \\
\text { Total time: } 30 \text { days } \\
\text { Temperature: } 30{ }^{\circ} \mathrm{C} \\
\text { Speed: } 200 \mathrm{rpm} \\
\text { pH: } 4.5\end{array}$ & 1 & $>99$ & 94 & $\begin{array}{l}\text { (Rodarte-Morales } \\
\text { et al., 2012a) }\end{array}$ \\
\hline & Spiked water & & $\begin{array}{l}\text { Stirred tank } \\
\text { (Continuous) }\end{array}$ & $\begin{array}{l}\text { Reactor volume: } 1.5 \mathrm{~L} \\
\text { Total time: } 50 \text { days } \\
\text { Temperature: } 30^{\circ} \mathrm{C} \\
\text { pH: } 4.5\end{array}$ & 1000 & 95 & & $\begin{array}{c}\text { (Rodarte-Morales } \\
\text { et al., 2012b) }\end{array}$ \\
\hline & $\begin{array}{l}\text { Hospital } \\
\text { wastewater }\end{array}$ & $\begin{array}{l}\text { Trametes } \\
\text { versicolor }\end{array}$ & $\begin{array}{l}\text { Fluidized bed } \\
\text { (Continuous) }\end{array}$ & $\begin{array}{l}\text { Reactor volume: } 10 \mathrm{~L} \\
\text { Total time: } 8 \text { days } \\
\text { Temperature: } 25^{\circ} \mathrm{C} \\
\text { pH: } 4.5\end{array}$ & 1.62 & 100 & & $\begin{array}{l}\text { (Cruz-Morató et } \\
\text { al., 2014) }\end{array}$ \\
\hline & $\begin{array}{l}\text { Synthetic } \\
\text { wastewater }\end{array}$ & & $\begin{array}{l}\text { Membrane } \\
\text { Bioreactor } \\
\text { (Continuous) }\end{array}$ & $\begin{array}{l}\text { Reactor volume: } 5.5 \mathrm{~L} \\
\text { Total time: } 110 \text { days } \\
\text { Temperature: } 27^{\circ} \mathrm{C} \\
\text { pH: } 4.5\end{array}$ & 5 & 95 & $\begin{array}{l}\text { (Nguyen et al., } \\
\text { 2013) }\end{array}$ & \\
\hline Phenazone & $\begin{array}{l}\text { Hospital } \\
\text { wastewater }\end{array}$ & $\begin{array}{l}\text { Trametes } \\
\text { versicolor }\end{array}$ & $\begin{array}{l}\text { Fluidized bed } \\
\text { (Continuous) }\end{array}$ & $\begin{array}{l}\text { Reactor volume: } 10 \mathrm{~L} \\
\text { Total time: } 8 \text { days } \\
\text { Temperature: } 25^{\circ} \mathrm{C} \\
\text { pH: } 4.5\end{array}$ & 0.497 & 96 & 15 & $\begin{array}{l}\text { (Cruz-Morató et } \\
\text { al., 2014) }\end{array}$ \\
\hline Propranolol & $\begin{array}{l}\text { Non-sterile urban } \\
\text { wastewater }\end{array}$ & $\begin{array}{l}\text { Trametes } \\
\text { versicolor }\end{array}$ & $\begin{array}{l}\text { Fluidized bed } \\
\text { (Batch-fed) }\end{array}$ & $\begin{array}{l}\text { Reactor volume: } 10 \mathrm{~L} \\
\text { Total time: } 8 \text { days } \\
\text { Temperature: } 25^{\circ} \mathrm{C} \\
\text { Speed: } 135 \mathrm{rpm} \\
\text { pH: } 4.5\end{array}$ & 0.06 & 100 & 1 & $\begin{array}{l}\text { (Cruz-Morató et } \\
\text { al., 2013) }\end{array}$ \\
\hline Sulfamethoxazole & $\begin{array}{l}\text { Hospital } \\
\text { wastewater }\end{array}$ & $\begin{array}{l}\text { Trametes } \\
\text { versicolor }\end{array}$ & $\begin{array}{r}\text { Fluidized bed } \\
\text { (Continuous) }\end{array}$ & $\begin{array}{l}\text { Reactor volume: } 10 \mathrm{~L} \\
\text { Total time: } 8 \text { days } \\
\text { Temperature: } 25^{\circ} \mathrm{C} \\
\text { pH: } 4.5\end{array}$ & 1.41 & 100 & 51.9 & $\begin{array}{l}\text { (Cruz-Morató et } \\
\text { al., 2014) }\end{array}$ \\
\hline Tetracycline & $\begin{array}{l}\text { Hospital } \\
\text { wastewater }\end{array}$ & $\begin{array}{l}\text { Trametes } \\
\text { versicolor }\end{array}$ & $\begin{array}{r}\text { Fluidized bed } \\
\text { (Continuous) }\end{array}$ & $\begin{array}{l}\text { Reactor volume: } 10 \mathrm{~L} \\
\text { Total time: } 8 \text { days } \\
\text { Temperature: } 25^{\circ} \mathrm{C} \\
\text { pH: } 4.5\end{array}$ & 0.011 & 0 & & $\begin{array}{l}\text { (Cruz-Morató et } \\
\text { al., 2014) }\end{array}$ \\
\hline
\end{tabular}

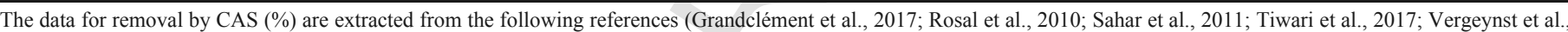
2015; Zuehlke et al., 2006).

${ }^{\text {a }}$ Conventional activated sludge.

cussed and the most observed TPs for widely-used PhACs are illustrated in Table 4.

There are several instruments for investigation of degradation products. Direct inlet-mass spectrometry (DI-MS, electron impact), gas chromatography-mass spectrometry (Hata et al., 2010a, 2010b; Rodarte-Morales et al., 2012b), ${ }^{1} \mathrm{H}$ nuclear magnetic resonance $\left({ }^{1} \mathrm{H}\right.$ $\mathrm{NMR}$ ) and ${ }^{13} \mathrm{C}$ NMR (Hata et al., 2010a; Marco-Urrea et al., 2010b; Marco-Urrea et al., 2009) are the widely used instruments for identification of TPs. Liquid chromatography electrospray time-of-flight mass spectrometry (LC-ESI-TOF-MS) in negative and positive mode (Eibes et al., 2011; Stadlmair et al., 2017) and high-performance liquid chromatography-diode array detection-electrospray ionization mass spectrometry (HPLC-DAD-MS) are also used for compounds that are not possible to be handled by gas chromatography (Marco-Urrea et al., 2010c; Schwarz et al., 2010). Recently, newly developed and rapid laser diode thermal desorption-mass spectroscopy (LDTD-MS) was used for identification of TPs (Lonappan et al., 2017).

To detect the formation of toxic TPs, most researchers used a standard procedure called bioluminescence inhibition test or Microtox assay (ISO 11348-3, 2007), that employs bacterium Aliivibrio fis- cheri (Becker et al., 2016; Gros et al., 2014; Jelic et al., 2012; Marco-Urrea et al., 2010a, 2010b) or Photobacterium phosphoreum (Marco-Urrea et al., 2009). They are marine luminescent bacteria that emit visible light $\left(\lambda_{\max }=490 \mathrm{~nm}\right)$. Effluent toxicity is expressed as units of Equitox $/ \mathrm{m}^{3}$ (determined as $100 / \mathrm{EC}_{50}$ ). The Equitox values are directly proportional to toxicity level, while $\mathrm{EC}_{50}$ is inversely related to toxicity. Counting the number of living freshwater crustacean Thamnocephalus platyurus and defining the relative acute lethal toxicity (\%) as the percentage of lethality of fungal-treated influent compared to that of untreated sample is another method employed by Hata et al. (2010a). In addition, the toxicity of treated and untreated solutions can be assessed based on their inhibitory effect towards the growth of Gram-positive and Gram-negative bacterial species e.g. Bacillus subtilis, Bacillus megaterium, Escherichia coli, and Saccharomyces. In this case, microorganisms are exposed to the media and the number of viable cells is monitored over a period of time (Becker et al., 2016; Rahmani et al., 2015). Moreover, the Yeast Estrogen Screen assay (YES) can confirm the estrogenic activity of environmental samples, such as wastewater effluent (Spengler et al., 2001). In this assay, the human estrogen receptor (hER) is expressed in yeast to make it re- 
Table 4

Structures of most studied micropollutants and proposed transformation products formed during biodegradation.

\begin{tabular}{|c|c|c|c|c|c|}
\hline Compounds & Acronym & $\begin{array}{l}\text { Compound } \\
\text { mass }(\mathrm{Da})\end{array}$ & Formula & Chemical structure & 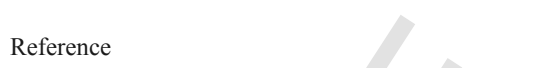 \\
\hline Carbamazepine & $\mathrm{CBZ}$ & 236.269 & $\mathrm{C}_{15} \mathrm{H}_{12} \mathrm{~N}_{2} \mathrm{O}$ & & $\begin{array}{l}\text { (Golan-Rozen et al., 2011; Golan-Rozen et al., 2015; } \\
\text { Hata et al., 2010b; Jelic et al., 2012) }\end{array}$ \\
\hline 10,11-epoxycarbamazepine & CBZ-EP & 253.0983 & $\mathrm{C}_{15} \mathrm{H}_{12} \mathrm{~N}_{2} \mathrm{O}_{2}$ & & \\
\hline 10,11-dihydroxycarbamazepine & $\mathrm{DiOH}-\mathrm{CBZ}$ & 293.091 & $\mathrm{C}_{15} \mathrm{H}_{14} \mathrm{~N}_{2} \mathrm{O}_{3}$ & & \\
\hline $\begin{array}{l}\text { 2-and 3-and 4-hydroxy } \\
\text { carbamazepine }\end{array}$ & $\begin{array}{l}\text { 2-OH-CBZ } \\
\text { 3-OH- } \\
\text { CBZ } \\
4-\mathrm{OH}- \\
\text { CBZ }\end{array}$ & 252.27 & $\mathrm{C}_{15} \mathrm{H}_{12} \mathrm{~N}_{2} \mathrm{O}_{2}$ & & \\
\hline Acridone & Acridone & 196.0762 & $\mathrm{C}_{13} \mathrm{H}_{9} \mathrm{NO}$ & & \\
\hline Acridine & Acridine & 179.222 & $\mathrm{C}_{13} \mathrm{H}_{9} \mathrm{~N}$ & & \\
\hline Diclofenac & DCF & 296.149 & $\mathrm{C}_{14} \mathrm{H}_{11} \mathrm{Cl}_{2} \mathrm{NO}_{2}$ & & $\begin{array}{l}\text { (Eibes et al., 2011; Hata et al., 2010a; Lonappan et } \\
\text { al., 2017; Marco-Urrea et al., 2010b) }\end{array}$ \\
\hline 4,5-dihydroxydiclofenac & $\begin{array}{l}4^{\prime}, 5-\mathrm{diOH}- \\
\text { DCF }\end{array}$ & 328.147 & $\mathrm{C}_{14} \mathrm{H}_{11} \mathrm{Cl}_{2} \mathrm{NO}_{4}$ & & \\
\hline 4'-hydroxydiclofenac & $\begin{array}{l}4^{\prime}-\mathrm{OH}- \\
\text { DCF }\end{array}$ & 312.148 & $\mathrm{C}_{14} \mathrm{H}_{11} \mathrm{Cl}_{2} \mathrm{NO}_{3}$ & & \\
\hline 5-hydroxydiclofenac & 5-OH-DCF & 312.148 & $\mathrm{C}_{14} \mathrm{H}_{11} \mathrm{Cl}_{2} \mathrm{NO}_{3}$ & & \\
\hline Ciprofloxacin & $\mathrm{CPF}$ & 331.341 & $\mathrm{C}_{17} \mathrm{H}_{18} \mathrm{FN}_{3} \mathrm{O}_{3}$ & & $\begin{array}{l}\text { (Parshikov et al., 2001; Parshikov et al., 1999; Prieto } \\
\text { et al., 2011) }\end{array}$ \\
\hline Ciprofloxacin-7-ethylenediamine & CPF-1 & 305.309 & $\mathrm{C}_{15} \mathrm{H}_{16} \mathrm{FN}_{3} \mathrm{O}_{3}$ & & \\
\hline Ciprofloxacin N-Oxide & CPF-3 & 347.346 & $\mathrm{C}_{17} \mathrm{H}_{18} \mathrm{FN}_{3} \mathrm{O}_{4}$ & & \\
\hline $\mathrm{N}$-acetylciprofloxacin & $\begin{array}{l}\text { N-acetyl- } \\
\text { CPF }\end{array}$ & 373 & $\mathrm{C}_{19} \mathrm{H}_{20} \mathrm{FN}_{3} \mathrm{O}_{5}$ & & \\
\hline
\end{tabular}


Table 4 (Continued)

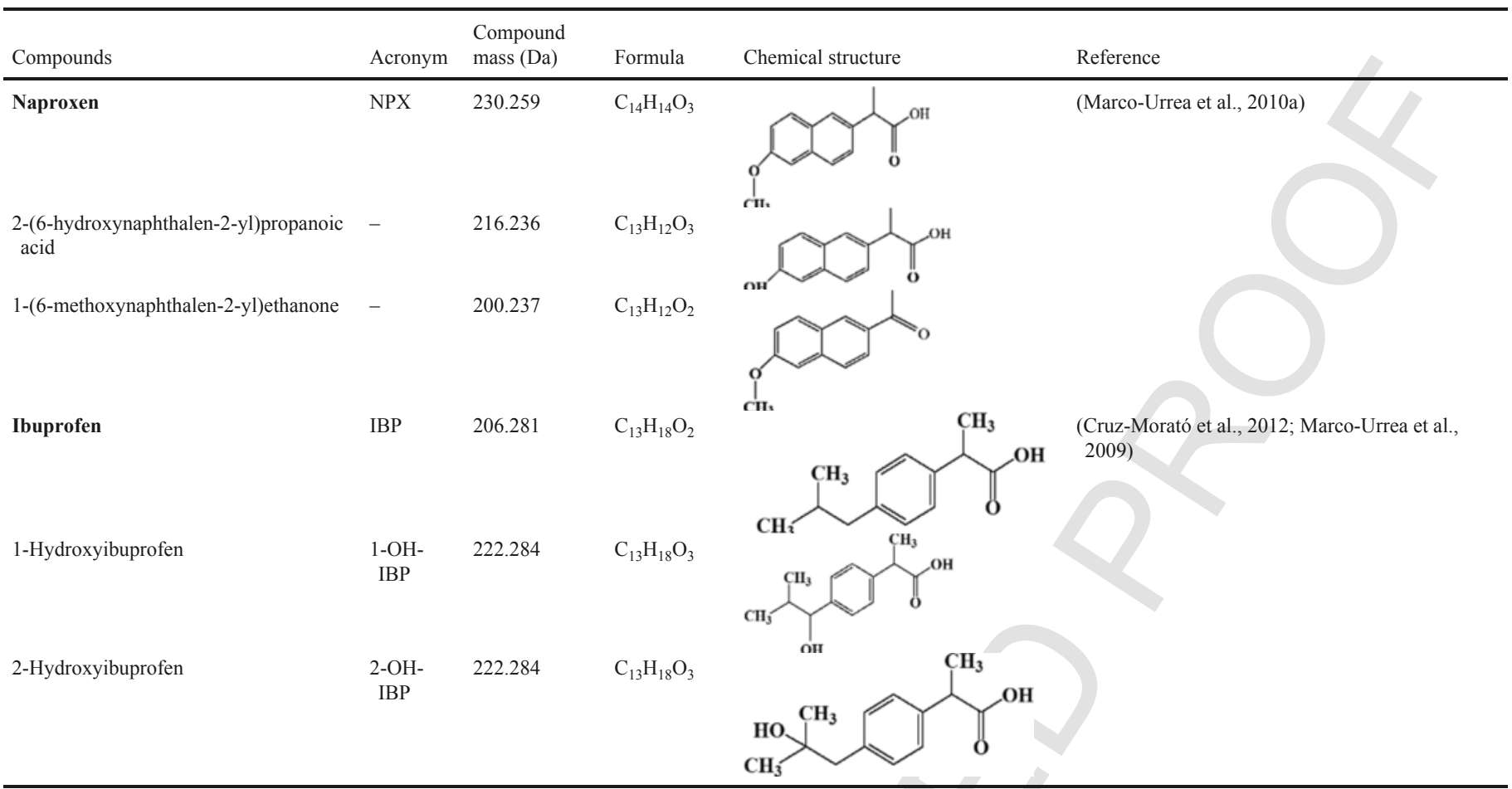

sponsive against estrogens (Routledge and Sumpter, 1996). The recombinant yeast hosts plasmids carrying lac-Z (the b-galactosidase-encoding reporter gene). In the presence of estrogenic compounds, the lac-Z gene is activated and b-galactosidase degrades a specific substrate that causes a color change from yellow to red as an indicator of compound estrogenicity (Bistan et al., 2012).

Fungal mediated degradation of DCF starts with the introduction of the hydroxyl group in its structure and formation of hydroxy diclofenac. This reaction facilitates further biodegradation (Hata et al., 2010a; Marco-Urrea et al., 2010b). In vitro and in vivo experiments using purified Lac and the CYP450 inhibitor, suggested that TV employed two different mechanisms to initiate degradation of DCF. Two TPs namely 4'-hydroxydiclofenac and 5-hydroxydiclofenac (Table 4) were identified which disappeared in $24 \mathrm{~h}$ resulting in a decrease in ecotoxicity according to Microtox test (Marco-Urrea et al., 2010b). Hata et al. reported that DCF degradation by fungus PS produced the hydroxylated metabolites that were found in the degradation by TV, and also they found 4,5-dihydroxydiclofenac as transformation product (Hata et al., 2010b). These hydroxylated products disappear at the end of the treatment with decreasing trend in toxicity that suggests mineralization (Eibes et al., 2011). However, according to Stadlmair et al., polymerization occurred after degradation of DCF by horseradish peroxidase (HRP). TPs showed lower toxicity compared to the parent compound (Stadlmair et al., 2017).

Hydroxylation is also the predominant start point for conversion of IBP to its TPs. In degradation of IBP by TV, it was reported to transform to 1-hydroxy ibuprofen and 2-hydroxy ibuprofen intermediates. These species were finally transformed to 1,2-dihydroxy ibuprofen (Table 4) during 7 days of incubation. However, Microtox bioassay revealed an increase in the toxicity after 7 days which was related to the presence of 1,2-hydroxy ibuprofen (Marco-Urrea et al., 2009). This finding emphasizes the significance of the identification of TPs in any treatment since they might be more toxic than their original compound (Cruz-Morató et al., 2012). Likewise, hydroxyla- tion reaction played an important role in the degradation of KEP with TV. 2-[3-(4-hydroxybenzoyl)phenyl]-propanoic acid, 2-[(3-hydroxy(phenyl)methyl)phenyl]-propanoic acid and 2-(3-benzyl-4-hydroxyphenyl)-propanoic acid were detected as main intermediates of TV activity. However, none of the mentioned intermediates was detected at the final stage which suggested KEP mineralization. It was also observed that extracellular enzyme (Lac) had a negligible effect on the degradation of KEP (Marco-Urrea et al., 2010c). Hata et al. found four hydroxylated TPs in degradation of MFA by PS. Their results showed that CYP450 catalyzed the hydroxylation which finally resulted in complete removal of acute lethal toxicity of MFA after 6 days of treatment (Hata et al., 2010b).

Both CYP450 and Lac can mediate the degradation of NPX in whole-cell WRF treatment. 1-(6-methoxynaphthalen-2-yl) ethanone and 2-(6-hydroxynaphthalen-2-yl) propanoic acid were detected as intermediates of NPX which disappear after $6 \mathrm{~h}$ of incubation without remaining toxicity (Marco-Urrea et al., 2010a). Also, 6-O-desmethyl-naproxen, was reported as the major degradation products of the NPX in a bioreactor of PC (Rodarte-Morales et al., 2012b).

Degradation pathways of CBZ by whole-cell WRFs, such as TV and PO was reported to result in the formation of 10,11-dihydro-10,11-epoxycarbamazepine while pure Lac resulted in the formation of $9(10 \mathrm{H})$-acridone as TPs after $48 \mathrm{~h}$ (Hata et al., 2010b). Microtox test showed that the toxicity of these two TPs is higher than the toxicity of CBZ (Jelic et al., 2012). Similarly, fungi other than WRFs, such as Umbelopsis ramanniana and Cunninghamella elegans produce 10,11-epoxycarbamazepine as the major TPs but they also produce (2-and 3-hydroxy carbamazepine) (Kang et al., 2008). Extracellular MnP and intracellular CYP450 were identified to affect the $\mathrm{CBZ}$ oxidation. It is noteworthy that at high initial CBZ concentration (10 mg/L), 10,11-epoxycarbamazepine was the major stable TP, but at an environmentally relevant concentration $(1 \mu \mathrm{g} / \mathrm{L})$, further transformation of 10,11-epoxycarbamazepine to 10,11 trans-diol was carried out by PO fungus (Golan-Rozen et al., 2011). It seems that the 
major TP of CBZ degradation with fungi is 10,11- epoxycarbamazepine though other TPs, especially hydroxylated derivatives were also identified. However, the toxicity of the TPs seemed to be more than CBZ.

Sulfonamides are sometimes desulfonated as a result of biodegradation with WRFs (García-Galán et al., 2011). For example, aniline and 4-(2-imino-1-pyridyl)aniline were identified as TPs of SPY and 4-(6-imino-2,4-dimethoxypyrimidin-1-yl)aniline was determined for SDM (Schwarz et al., 2010). Also, desamino-sulfamethazine and hydroxyl-sulfamethazine were identified for SMZ and for the transformation of SPY and STZ, a formyl intermediate was observed after the loss of the thiazole/pyrimidine group (García-Galán et al., 2011; Rodríguez-Rodríguez et al., 2012). In another study, anions, such as sulfate, nitrate, and nitrite were detected as an intermediate of SMX degradation with crude VP obtained from Bjerkandera adusta (Eibes et al., 2011). Rahmani et al. showed that the growth inhibition property of a solution containing SMX and STZ against bacteria was remarkably decreased after treatment with laccase (Rahmani et al., 2015). Although many metabolites of sulfonamides degradation with enzymes were identified, the pathways and mechanisms still need to be studied.

In the case of CPF degradation with Gloeophyllum striatum after $90 \mathrm{~h}$, reduction in antibacterial activity and production of $\mathrm{CO}_{2}$ was reported along with eleven metabolites including hydroxylated congeners and TPs indicating the degradation of the piperazinyl moiety (Wetzstein et al., 1999). Using extracted Lac resulted in the identification of new TPs that were obtained from the breakdown of piperazinyl moiety, hydroxylation, and removal of a cyclopropyl group (Prieto et al., 2011). There are also other acetylated derivatives identified as TPs for degradation of CPF using different fungi, such as Pestalotiopsis guepini (Parshikov et al., 2001), Mucor ramannianus (Parshikov et al., 1999) and Trichoderma viride (Parshikov et al., 2002). Similarly, in degradation of EFC by Gloeophyllum striatum hydroxylated congeners, an isatin-type compound (obtained by cleavage of the heterocyclic core of EFC) and an anthranilic acid derivative was identified (Wetzstein et al., 1997). TPs with hydroxylated aromatic rings undergo ring cleavage to transform to one catechol-type and four potential oxidizable $o$-aminophenol intermediates (Wetzstein et al., 2006). Degradation of EFC with Mucor ramannianus, resulted in the formation of EFC N-oxide, $\mathrm{N}$-acetylciprofloxacin, and desethylene-enrofloxacin as TPs (Parshikov et al., 2000). Parshikov et al. demonstrated that the intermediates and TPs of degradation of NOR with Pestalotiopsis guepini and Trichoderma viride were analogous to those derived from CPF by the same fungi (Parshikov et al., 2001, 2002). Gros et al. studied the degradation of antibiotic ofloxacin by TV in sterile and unsterile hospital wastewater as well as synthetic wastewater. They reported that TPs of ofloxacin are obtained mainly through hydroxylation, oxidation, and cleavage of the piperazine ring. Their toxicity tests showed a reduction of the toxicity in the synthetic medium and in the batch bioreactor (Gros et al., 2014). Llorca et al. investigated the TPs formed by enzymatic degradation of antibiotic TC. They attributed the formation of major TPs to (bi) demethylation, dehydroxylation and oxidation of the rings $\mathrm{C}$ and A (Llorca et al., 2015).

To sum up, few researchers have paid attention to the evaluation of toxicity of TPs until the date and it should be considered in future investigations. According to the few published research works, in some cases, the TPs of enzymatic processes were found to be more toxic than their parent compounds. Therefore, the major TPs of enzymatic processes should be identified for the majority of present compounds in the waste streams and their toxicity should be determined prior to the decision for system scale-up.

\subsection{Crude enzyme}

Extraction of enzymes from microorganisms and using them instead of using live cultures for removal of pollutant from aqueous media has several advantages. Extracted enzymes do not need the continuous addition of nutrients or compete with bacteria and they can reach high reaction kinetics in mild temperature and $\mathrm{pH}$ conditions (Baldrian, 2006; De Cazes et al., 2014a; Demarche et al., 2012). Enzymatic treatment consumes less energy and chemicals and produce fewer wastes compared to other bioprocesses (Grandclément et al., 2017; Jochems et al., 2011). Enzymatic treatment is particularly an attractive technology for the treatment of PhACs that are resistant to conventional treatment. The performance of individual ligninolytic enzymes has been studied for the removal of a broad range of micropollutants and the results are summarized in Table 5. In addition, the capacity of crude and purified extracellular ligninolytic enzymes for PhACs removal in batch and continuous mode has been extensively investigated (Nguyen et al., 2014a, 2014b, 2014c, 2015; Tran et al., 2010; Yang et al., 2013c). For instance, Li et al. reported more than $90 \%$ removal of NPX in two days of reaction with crude enzyme obtained from PC at an initial concentration of $10 \mathrm{mg} / \mathrm{L}$. This level of degradation efficiency was higher than the performance in whole-cell cultivation in which $68 \%$ removal efficiency was achieved after two days (Li et al., 2015). Margot et al. compared the ability of extracted Lac from bacterium Streptomyces cyaneus and TV for degradation of DCF and MFA. They reported that fungal Lac was more active than bacterial Lac in normal conditions of municipal wastewater (neutral $\mathrm{pH}$ and $10-25^{\circ} \mathrm{C}$ ) and showed faster kinetics for degradation of DCF and MFA. Complete removal of DCF and MFA was achieved during 12 days of incubation with fungal Lac, while around $50 \%$ of both pollutants were removed by bacterial Lac within the same incubation time (Margot et al., 2013a). Llorca et al. reported that degradation efficiency of Lac towards TC after $18 \mathrm{~h}$ and EreB esterase towards ETM after $16 \mathrm{~h}$ were $\sim 78 \%$ and $\sim 50 \%$, respectively (Llorca et al., 2015).

\subsubsection{Purified enzyme}

Purification of the enzyme is a costly process that can be performed through different methods, such as membrane separation, size exclusion chromatography, etc. (Lloret et al., 2010). Purified oxidoreductase enzymes, obtained from different strains of WRF, have been used for removal of pollutants from aqueous media in both continuous and batch reactors (Marco-Urrea et al., 2010b). Purified oxidoreductase enzymes demonstrated degradation potential towards a wide range of micropollutants, however, crude enzyme demonstrated better removal performance for some compounds, such as NPX and DCF (Table 5). It was related to the natural mediators that exist in the crude enzyme (Wang and Wang, 2016). For instance, Tran et al. observed complete removal ( $>99 \%$ ) of several compounds, such as IBP, DCF and IDM and related them to the natural mediators in crude Lac obtained from TV grown in basal liquid medium (Tran et al., 2010) whereas purified laccase obtained from TV and Aspergillus oryzae achieved only 20-50\% removal efficiency for these compounds (Lloret et al., 2010; Nguyen et al., 2015). Although utilization of crude enzyme is more economical and in some cases leads to higher removal efficiency compared to the purified enzyme, the crude solution contains remarkable levels of the unspent nutrients that can increase the organic loading of wastewater to be treated (Nguyen et al., 2016b). Therefore, still more research is needed to simultaneously take advantage of natural mediators and rejection of nutrients. 
Table 5

Biodegradation of PhACs by crude and purified enzymes.

\begin{tabular}{|c|c|c|c|c|c|c|}
\hline Compound & Enzyme source & Condition & $\begin{array}{l}\text { Concentration of } \mathrm{PhACs}^{\mathrm{a}} \\
(\mathrm{mg} / \mathrm{L})\end{array}$ & Scale & $\begin{array}{l}\text { Conversion } \\
(\%)\end{array}$ & References \\
\hline Acetaminophen & Not mentioned & Purified laccase & 7.55 & $25^{\circ} \mathrm{C}, 47 \mathrm{~min}, 50 \mathrm{~mL}$ & 50 & (Lu et al., 2009) \\
\hline \multirow[t]{3}{*}{ Carbamazepine } & Trametes versicolor & Crude laccase & 0.01 & $\begin{array}{l}30^{\circ} \mathrm{C}, 125 \mathrm{rpm} \\
48 \mathrm{~h}, 100 \mathrm{~mL}\end{array}$ & 37 & (Tran et al., 2010) \\
\hline & $\begin{array}{l}\text { Phanerochaete } \\
\text { chrysosporium }\end{array}$ & Crude lignin peroxidases & 5 & $\begin{array}{l}25^{\circ} \mathrm{C}, 120 \mathrm{rpm}, \\
2 \mathrm{~h}, 10 \mathrm{~mL}\end{array}$ & $<10$ & (Zhang and Geißen, 2010) \\
\hline & $\begin{array}{l}\text { Phanerochaete } \\
\text { chrysosporium }\end{array}$ & $\begin{array}{l}\text { Purified manganese } \\
\text { peroxidases }\end{array}$ & 4.7 & $\begin{array}{c}30^{\circ} \mathrm{C}, 150 \mathrm{rpm} \\
24 \mathrm{~h}, 100 \mathrm{~mL}\end{array}$ & 14 & (Hata et al., 2010b) \\
\hline \multirow[t]{5}{*}{ Diclofenac } & Trametes versicolor & Purified laccase & 10 & $\begin{array}{c}25^{\circ} \mathrm{C}, 135 \mathrm{rpm} \\
4.5 \mathrm{~h}, 25 \mathrm{~mL}\end{array}$ & 95 & (Marco-Urrea et al., 2010b) \\
\hline & Bjerkandera adusta & $\begin{array}{l}\text { Purified versatile } \\
\text { peroxidase }\end{array}$ & 2.5 & $22^{\circ} \mathrm{C}, 25 \mathrm{~min}, 50 \mathrm{~mL}$ & 100 & (Eibes et al., 2011) \\
\hline & Trametes versicolor & Crude laccase & 0.01 & $\begin{array}{l}30^{\circ} \mathrm{C}, 125 \mathrm{rpm}, 48 \mathrm{~h}, \\
100 \mathrm{~mL}\end{array}$ & 100 & (Tran et al., 2010) \\
\hline & $\begin{array}{l}\text { Myceliophthora } \\
\text { thermophila }\end{array}$ & Purified laccase & 5 & $\begin{array}{l}22{ }^{\circ} \mathrm{C}, 8 \mathrm{~h} \\
20 \mathrm{~mL}\end{array}$ & 65 & (Lloret et al., 2010) \\
\hline & $\begin{array}{l}\text { Phanerochaete } \\
\text { chrysosporium }\end{array}$ & Crude lignin peroxidases & 5 & $\begin{array}{l}25^{\circ} \mathrm{C}, 120 \mathrm{rpm}, \\
2 \mathrm{~h}, 10 \mathrm{~mL}\end{array}$ & 100 & (Zhang and Geißen, 2010) \\
\hline Ibuprofen & Trametes versicolor & Crude laccase & 0.01 & $\begin{array}{l}30^{\circ} \mathrm{C}, 125 \mathrm{rpm}, 48 \mathrm{~h}, \\
100 \mathrm{~mL}\end{array}$ & 38 & (Tran et al., 2010) \\
\hline \multirow[t]{2}{*}{ Naproxen } & Trametes versicolor & Purified laccase & 20 & $\begin{array}{l}25^{\circ} \mathrm{C}, 135 \mathrm{rpm} \\
30 \mathrm{~h}, 25 \mathrm{~mL}\end{array}$ & 10 & (Marco-Urrea et al., 2010a) \\
\hline & & Crude laccase & 0.01 & $\begin{array}{l}30^{\circ} \mathrm{C}, 125 \mathrm{rpm}, 48 \mathrm{~h}, \\
100 \mathrm{~mL}\end{array}$ & 100 & (Tran et al., 2010) \\
\hline Sulfadimethoxine & Trametes versicolor & Purified laccase & 310.33 & $\begin{array}{l}21^{\circ} \mathrm{C}, \text { Static, } \\
15 \mathrm{~d}, 100 \mathrm{~mL}\end{array}$ & 75.1 & (Schwarz et al., 2010) \\
\hline Sulfanilamide & Trametes versicolor & Purified laccase & 172.20 & $\begin{array}{l}21^{\circ} \mathrm{C}, \text { Static, } \\
15 \mathrm{~d}, 100 \mathrm{~mL}\end{array}$ & 10 & (Schwarz et al., 2010) \\
\hline \multirow[t]{2}{*}{ Sulfapyridine } & Trametes versicolor & Purified laccase & 10 & $\begin{array}{c}25^{\circ} \mathrm{C}, 135 \mathrm{rpm}, \\
50 \mathrm{~h}, 50 \mathrm{~mL}\end{array}$ & 75 & $\begin{array}{l}\text { (Rodríguez-Rodríguez et al., } \\
\text { 2012) }\end{array}$ \\
\hline & & & 249.29 & $21^{\circ} \mathrm{C}$, Static, & 95.6 & (Schwarz et al., 2010) \\
\hline Sulfathiazole & Trametes versicolor & Purified laccase & 10 & $\begin{array}{l}25^{\circ} \mathrm{C}, 135 \mathrm{rpm}, \\
50 \mathrm{~h}, 50 \mathrm{~mL}\end{array}$ & 82 & $\begin{array}{l}\text { (Rodríguez-Rodríguez et al., } \\
\text { 2012) }\end{array}$ \\
\hline
\end{tabular}

${ }^{a}$ Pharmaceutically active compounds.

\subsubsection{Mediator effect}

Lac catalyzes the mono-electronic oxidation of PhACs through copper active sites. However, the oxidation-reduction potential (ORP) of the enzyme affects the extent of removal (D'Acunzo et al., 2006). Poor degradation of non-phenolic PhACs is generally attributed to the presence of strong electron withdrawing groups (EWGs), such as amide $\left(-\mathrm{CONR}_{2}\right)$, carboxylic $(-\mathrm{COOH})$, halogen $(-\mathrm{X})$ and nitro $\left(-\mathrm{NO}_{2}\right)$ in the molecular structure and higher ORP of non-phenolic compounds compared to Lac (D'Acunzo et al., 2006).

Degradation efficiency of pollutants with Lac can be enhanced by the addition of mediators that work as electron shuttles between the target compounds and enzyme (Kim and Nicell, 2006). The low molecular weight mediators, such as syringaldazine (SA) and 1-hydroxybenzotriazole (HBT) are oxidized by the enzyme, they diffuse and oxidize the substrate that cannot enter the enzymatic pocket due to its size. The generated radicals serve as a shuttle for electron transfer between PhACs and Lac and consequently facilitate the degradation of recalcitrant compounds. Also, they can enhance the degradation of non-phenolic compounds by generating highly reactive radicals as a result of mediator oxidation by the enzyme. Therefore, the mediator can extend the range of substrates degradable by the enzyme (Fabbrini et al., 2002). In Table 6, the most studied redox mediator for Lac with their structure and related information are listed. The mediators follow three mechanisms for oxidation i.e. ionic mechanisms, hydrogen atom transfer, and electron transfer (Asif et al., 2017). For instance, HBT and SA tend to follow hydrogen atom transfer, while 2,2'-azino-bis(3-ethylbenzothiazoline-6-sulphonic acid) (ABTS) and 2,2,6,6-tetramethylpiperidinyloxyl (TEMPO) were reported to follow electron transfer and ionic mechanisms, respectively (Ashe et al., 2016; Astolfi et al., 2005). The type and concentration of mediator and the properties of target compound affect the performance of a mediator. For instance, violuric acid (VLA) and HBT were reported to work better for non-phenolic PhACs, while SA and ABTS showed better performance for phenolic compounds (Nguyen et al., 2015, 2014b, 2016b; Yang, 2012).

Lloret et al. investigated the effects of SA on the degradation of DCF by commercial Lac from Myceliophthora thermophila (MT). They found that removal of DCF was improved from 40 to $80 \%$ by increasing the SA concentration from 0.1 to $0.5 \mathrm{mM}$ (Lloret et al., 2010). Similarly, Nguyen et al. obtained $35 \%$ improvement in the removal of DCF by increasing the concentration of SA from 0.01 to $0.1 \mathrm{mM}$ in an enzymatic reactor (Nguyen et al., 2016b). Increasing the mediator concentration beyond a threshold level, may not affect the removal of PhAC. For example, Ashe et al. reported that NPX can be efficiently removed by Lac in the presence of VA or HBT mediators but increasing the VA concentration from 0.5 to $1 \mathrm{mM}$ caused no improvement in the removal of NPX (Ashe et al., 2016). The small aminoxyl radicals formed by reaction of HBT and Lac can abstract hydrogen atom from the $\mathrm{O}-\mathrm{H}$ bond in substrates and form the phenoxyl radicals which are able to react with the substrate (Coniglio et al., 2008). Suda et al. investigated the degradation of several tetracycline antibiotics (TC, CTC, DC, and OTC) with a Lac mediated by HBT. Their results showed complete elimination of DC and CTC in $15 \mathrm{~min}$, and complete removal of CTC and TC in $1 \mathrm{~h}$ (Suda et al., 2012). Nguyen et al. observed that coupling HBT with laccase in a fungal membrane bioreactor (MBR) can eliminate DCF (70-95\%) and NPX (20-98\%) that are resistant to bacterial degradation but can- 
Table 6

Physicochemical properties of redox-mediators used to improve the performance of laccase-based treatment of PhACs.

\begin{tabular}{|c|c|c|c|c|c|c|c|c|}
\hline $\begin{array}{l}\text { Redox- } \\
\text { mediator }\end{array}$ & $\begin{array}{l}\text { Natural/ } \\
\text { synthetic }\end{array}$ & Type of mediator & Free radicals & $\begin{array}{l}\text { Oxidation } \\
\text { mechanism }\end{array}$ & Chemical structure & $\begin{array}{l}\text { Application } \\
\text { for PhACs } \\
\text { removal }\end{array}$ & $\begin{array}{l}\text { Average } \\
\text { removal (\%) }\end{array}$ & References \\
\hline \multirow[t]{2}{*}{$\mathrm{HBT}^{\mathrm{A}}$} & Synthetic & $\mathrm{N}-\mathrm{OH}$ & $\begin{array}{l}\text { Aminoxyl } \\
=\mathrm{N}-\mathrm{O}^{\bullet}\end{array}$ & $\mathrm{HAT}^{\mathrm{H}}$ & & $\mathrm{NPX}^{\mathrm{K}}$ & 89 & $\begin{array}{l}\text { (Lloret et al., 2013; Marco-Urrea et al., } \\
\text { 2010a; Tran et al., 2010) }\end{array}$ \\
\hline & & & & & & $\begin{array}{l}\mathrm{DCF}^{\mathrm{L}} \\
\mathrm{CBZ}^{\mathrm{M}}\end{array}$ & $\begin{array}{l}96 \\
60\end{array}$ & $\begin{array}{l}\text { (Lloret et al., 2013; Nguyen et al., 2014a; } \\
\text { Tran et al., 2010) }\end{array}$ \\
\hline \multirow[t]{5}{*}{$\mathrm{ABTS}^{\mathrm{B}}$} & Synthetic & ABTS & $\begin{array}{l}\mathrm{ABTS}^{+\bullet} \\
\text { ABTS }^{++}\end{array}$ & $\mathrm{ET}^{\mathrm{I}}$ & & $\mathrm{DCF}$ & 100 & (Tran et al., 2010) \\
\hline & & & & & & NPX & 100 & (Tran et al., 2010) \\
\hline & & & & & & $\operatorname{IDM}^{\mathrm{N}}$ & 100 & (Tran et al., 2010) \\
\hline & & & & & & $\mathrm{SDM}^{\mathrm{O}}$ & 100 & (Weng et al., 2012) \\
\hline & & & & & & $\mathrm{SMM}^{\mathrm{P}}$ & 100 & (Weng et al., 2012) \\
\hline TEMPO $^{\mathrm{C}}$ & Synthetic & $\mathrm{N}-\mathrm{O}$ & $\begin{array}{l}\text { Oxoammonium } \\
\mathrm{N}=\mathrm{O}^{\bullet}\end{array}$ & Ionic $^{\mathrm{J}}$ & & NPX & 37 & (Ashe et al., 2016) \\
\hline $\mathrm{HPI}^{\mathrm{D}}$ & Synthetic & $\mathrm{N}-\mathrm{OH}$ & $\begin{array}{l}\text { Aminoxyl } \\
=\mathrm{N}-\mathrm{O}^{*}\end{array}$ & $\mathrm{HAT}^{\mathrm{H}}$ & & NPX & 38 & (Ashe et al., 2016) \\
\hline \multirow[t]{7}{*}{$\mathrm{SA}^{\mathrm{E}}$} & Natural & $\mathrm{C}_{6} \mathrm{H}_{4}(\mathrm{OH})\left(\mathrm{OCH}_{3}\right)$ & $\begin{array}{l}\text { Phenoxyl } \\
\mathrm{C}_{6} \mathrm{H}_{5} \mathrm{O}^{\bullet}\end{array}$ & $\mathrm{HAT}^{\mathrm{H}}$ & & DCF & 64 & $\begin{array}{l}\text { (Lloret et al., 2013; Nguyen et al., 2014a; } \\
\text { Sathishkumar et al., 2014) }\end{array}$ \\
\hline & & & & & & $\mathrm{IBP}^{\mathrm{Q}}$ & 19 & $\begin{array}{l}\text { (Nguyen et al., 2015; Nguyen et al., } \\
2016 b \text { ) }\end{array}$ \\
\hline & & & & & & $\mathrm{GFZ}^{\mathrm{R}}$ & 34 & (Nguyen et al., 2015) \\
\hline & & & & & & NPX & 23 & (Nguyen et al., 2015) \\
\hline & & & & & & $\mathrm{KEP}^{\mathrm{S}}$ & 17 & (Nguyen et al., 2015) \\
\hline & & & & & & CBZ & 32 & $\begin{array}{l}\text { (Nguyen et al., 2015; Nguyen et al., } \\
2014 \mathrm{c} \text { ) }\end{array}$ \\
\hline & & & & & & $\mathrm{AMP}^{\mathrm{T}}$ & 100 & $\begin{array}{l}\text { (Nguyen et al., 2015; Nguyen et al., } \\
\text { 2016b) }\end{array}$ \\
\hline $\mathrm{VLA}^{\mathrm{F}}$ & Natural & $\mathrm{N}-\mathrm{OH}$ & $\begin{array}{l}\text { Aminoxyl } \\
=\mathrm{N}-\mathrm{O}^{\bullet}\end{array}$ & $\mathrm{HAT}^{\mathrm{H}}$ & & SDM & 100 & (Weng et al., 2012) \\
\hline $\mathrm{VAN}^{\mathrm{G}}$ & Synthetic & $\mathrm{C}_{6} \mathrm{H}_{4}(\mathrm{OH})\left(\mathrm{OCH}_{3}\right)$ & $\begin{array}{c}\text { Phenoxyl } \\
\mathrm{C}_{6} \mathrm{H}_{5} \mathrm{O}^{\bullet}\end{array}$ & $\mathrm{HAT}^{\mathrm{H}}$ & & $\begin{array}{l}\text { SMM } \\
\text { NPX }\end{array}$ & $\begin{array}{l}100 \\
39\end{array}$ & $\begin{array}{l}\text { (Weng et al., 2012) } \\
\text { (Ashe et al., 2016) }\end{array}$ \\
\hline
\end{tabular}

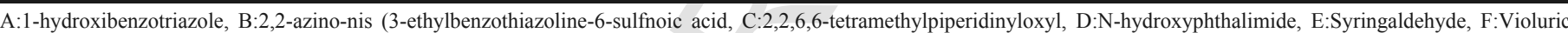

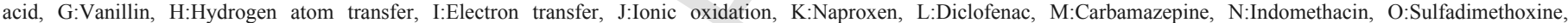
P:Sulfamonomethoxine, Q:Ibuprofen, R:Gemfibrozil, S:Ketoprofen T:Amitriptyline.

not degrade compounds such as IBP, GFZ and AMP that are perfectly removed by activated sludge treatment (Nguyen et al., 2013). This finding indicates that WRF and activated sludge would be a complementary system in WWTPs. However, the high loading of mediator required for the treatment casts doubts on the applicability of enzyme-mediator systems. Margot et al. investigated the removal of SMX with Lac mediated by ABTS, SA, and acetosyringone (ACE). They observed that mediators were consumed at the mediator to pollutant molar ratio of 1.1-16 (Margot et al., 2015).

Another issue with using mediators is the compromising of enzymatic activity after the addition of mediators though they can improve the kinetics of the reaction. For instance, Hata et al. reported $90 \%$ reduction in Lac activity $8 \mathrm{~h}$ after the addition of HBT (Hata et al., 2010 b). Likewise, rapid reduction in activity of laccase was reported after the addition of ABTS, VA, or HBT but the rate of inactivation depends on the stability of the generated radicals (Ashe et al., 2016). If there is no enzyme inhibitor in the medium, rapid inactivation of enzyme in presence of mediator can be due to the blocking of enzyme active sites by metabolites and charged radicals and also the reaction of enzyme-active sites with metabolites and form non-productive complexes (Asif et al., 2017; Purich, 2010). Nevertheless, the periodic enzyme replenishment will be required to maintain the removal efficiency of PhACs which increases the operational cost.
Although the use of mediators can improve the removal of micropollutant, these compounds are toxic and their release into the environment may pose chronic problems (Grandclément et al., 2017). As a result, increasing the toxicity of treated wastewater with enzyme-mediator system leads to another environmental problem. For example, Nguyen et al. found that effluent of enzymatic treatment mediated with SA was more toxic than control sample for all dosages applied, while the addition of HBT did not increase the toxicity at concentrations lower than $0.5 \mathrm{mM}$ (Nguyen et al., 2014a). Therefore, to develop an effective removal strategy, selecting the appropriate mediator and determination of its optimum concentration are critical. Furthermore, it has to be ensured that use of mediator does not result in increasing the toxicity of the effluent.

\subsubsection{Operational parameters}

The performance of ligninolytic enzymes in wastewater treatment plants depends on operational conditions and physiochemical properties of PhACs and wastewater. Briefly, the properties of wastewater, such as temperature, $\mathrm{pH}$, salinity and the presence of metals and dissolved organic/inorganic matter may influence the performance of fungal cultures or their extracted enzyme (Yang et al., 2013b). In the following sections, the effects of different parameters have been summarized. 
The temperature of wastewater affects both the stability of biocatalytic systems and the rate of reaction. It is assumed that the reaction rate increases to some extent when the temperature is increased (Zhang and Geißen, 2010). However, depending on the strain of fungi, thermal denaturation of enzymes is expected at a temperature higher than $40{ }^{\circ} \mathrm{C}$ (Mukhopadhyay et al., 2015; Sampaio et al., 2016). Few studies investigated the effect of temperature on the activity of ligninolytic enzymes (Bosco et al., 2002; Wen et al., 2010). The optimal temperature to obtain the highest degradation efficiency of ligninolytic enzymes differ from one compound to another. For instance, Wen et al. studied the degradation of TC and OTC by crude LiP obtained from $\mathrm{PC}$ and observed that in the range of $30-37^{\circ} \mathrm{C}$, TC was totally removed while the degradation efficiency of OTC was about $90 \%$ at $30{ }^{\circ} \mathrm{C}$ and increased with the temperature, until it was $37^{\circ} \mathrm{C}$ (Wen et al., 2009). In a related study, Margot et al. used purified Lac from TV to degrade DCF and observed that by increasing the temperature from $10^{\circ} \mathrm{C}$ to $25^{\circ} \mathrm{C}$, degradation efficiency was increased and further temperature increase resulted in a plateau (Margot et al., 2013b). Similarly, Naghdi et al. showed that Lac obtained from TV has its highest stability at $30{ }^{\circ} \mathrm{C}$ with $66 \%$ of its initial activity and between 50 and $70{ }^{\circ} \mathrm{C}$, Lac could not retain more than $11 \%$ of its initial activity (Naghdi et al., 2017).

The performance of enzyme and several properties of substrates can be highly impacted by $\mathrm{pH}$ of the reaction medium, which subsequently affects the extent of PhACs removal. The effects of $\mathrm{pH}$ on degradation efficiency are caused by the stability of target compounds at different $\mathrm{pH}$ levels and the $\mathrm{pH}$ dependency of the enzyme activity. The latter is because of the fact that the $\mathrm{pH}$ changes the ionization status of enzyme and each enzyme can be active only in a special ionization status (Wen et al., 2009).

The optimum $\mathrm{pH}$ for DCF removal (60-100\%) by purified Lac obtained from TV and MT and LiP obtained from PC was reported to be in the range of 3.0-4.5 (Lloret et al., 2010; Margot et al., 2013b; Nguyen et al., 2014b; Zhang and Geißen, 2010). Zhang and Geißen found that crude LiP obtained from PC can completely degrade DCF at $\mathrm{pH} 3.0-4.5$ while only $10 \%$ degradation happened at $\mathrm{pH} 6.0$. They indicated that this decline in removal efficiency was due to the inactivation of LiP at higher $\mathrm{pH}$ (Zhang and Geißen, 2010). Wen et al. studied the degradation of TC and OTC with crude MnP obtained from $\mathrm{PC}$ and observed that $\mathrm{pH}$ range of $2.96-4.80$ was the optimum range for treatment (Wen et al., 2010). In another study, it was found that $\mathrm{pH} 4.2$ was the optimum value for degradation of TC and OTC with LiP. They also observed no degradation for $\mathrm{pH}$ values below 2.8 or above 5.4. Interestingly, for $\mathrm{pH}$ values higher than 4.2 , the degradation efficiency of TC decreased more rapidly than that of OTC (Wen et al., 2009). In a related study, Weng et al. observed that Lac activity decreased when $\mathrm{pH}$ was pushed toward alkaline values and related it to the binding of hydroxide anion to the copper element of laccase, which subsequently interrupted the electron transfer pathway (Weng et al., 2013).

Besides temperature and $\mathrm{pH}$, the constituents of wastewater matrix, such as surfactants, natural organic matter, various organic/inorganic compounds and heavy metal ions need to be evaluated in the case of removal of PhACs (Hu et al., 2014). The effects of dissolved organic and inorganic compounds on the activity of Lac and removal of PhACs has been discussed by several studies. Accordingly, compounds such as heavy metals, halides, sulfides and natural/synthetic organics can inhibit the activity of Lac (D'Souza-Ticlo et al., 2009; Sondhi et al., 2014; Wan et al., 2015). Each compound may inhibit the enzyme via a different mechanism. For instance, fatty acids inhibit the catalytic potential of Lac by blocking the enzyme binding sites for phenolic substrates (Gianfreda et al., 1998). Also, the cat- alytic voltammetry analysis indicated that anionic inhibitors, such as sulfides and halides could block the access of substrates to the active copper sites in Lac (Blanford et al., 2009). Among anionic inhibitors, azide and fluoride are known as the most effective inhibitors that can rapidly reduce the activity of Lac by $50 \%$ even at very low concentrations (Bento et al., 2005). The inhibition of Lac by halides can follow this order: fluoride $>$ chloride $>$ bromide, however, the halides concentration required to inhibit Lac varies and no correlation with their inhibition potential have been found (Rodgers et al., 2010; Xu, 1996).

$\mathrm{Lu}$ et al. investigated the effect of natural organic matter on biodegradation of ACT with Lac and found that dissolved natural organic matter can inhibit self-coupling of the ACT and enhance its removal. It can be due to the cross-coupling between ACT and dissolved natural organic matter (Lu and Huang, 2009). Cross-coupling between pharmaceuticals and natural organic matter may play a more important role than self-coupling of pharmaceuticals in degradation of micropollutants due to a higher concentration of natural organic matter (Piccolo et al., 2000). Cross-coupling of the pollutants with molecules of natural organic matter through the oxidative action of enzymes can deactivate their biological effects. Also, the cross-coupling of natural organic matter molecules to each other as a result of enzymatic reaction can enhance the removal of natural organic matter. Therefore, using enzymatic treatment can simultaneously remove micropollutants and natural organic matter (Cozzolino and Piccolo, 2002; Piccolo et al., 2000).

Although oxidoreductase enzymes act non-specifically towards pollutants, they follow the "one electron oxidation" mechanism and therefore the substrates need to have electron donor properties in this reaction. The essential criteria for substrates of these enzymes are the presence of the heterocyclic or aromatic ring, easily oxidized substitutions, such as hydroxyl groups and electron donor substituent such as phenyl, alkyl, etc. (Tran et al., 2010). Hydrophobicity and the presence of electron donating groups (EDGs) or EWGs are important factors affecting the biodegradation of trace organic contaminants in WWTPs (Joutey et al., 2013). EWGs, such as an amide $\left(-\mathrm{CONR}_{2}\right)$ and carboxylic $(-\mathrm{COOH})$ groups cause the compounds to be less susceptible to oxidative catabolism while EDGs such as an amine $\left(-\mathrm{NH}_{2}\right)$ and hydroxyl $(-\mathrm{OH})$ groups facilitate the electrophilic attack by oxygenase produced in aerobic treatment (Tadkaew et al., 2011). As a consequence, high removal efficiency was observed for hydrophilic and hydrophobic compounds, which possess EDGs while low removal efficiency was observed for hydrophilic compounds bearing EWGs. It is noteworthy that some compounds, such as DCF and NPX contain both EDGs and EWGs and the overall influence of these groups on biodegradability is complex. In this case, an extensive study on the structure-activity relationship is required (D'Acunzo et al., 2006; Yang et al., 2013b).

\subsection{Immobilized enzyme}

Using batch reactors with free enzymes is not economically viable for wastewater treatment due to the high volume of wastewater to be treated, high quantities of required enzyme and necessity for removal of the enzyme at the end of treatment (Majeau et al., 2010). Since enzymes are expensive, the economic viability of the whole process needs to be demonstrated. To overcome the cost of large amount of free enzyme needed for real applications (due to enzyme losses during the treatment), different strategies that have been adopted include: (i) immobilization of the enzymes on supports to separate enzymes from the effluent and reuse them several times (Fernández-Fernández et al., 2013), (ii) using ultrafiltration membranes to prevent the release of enzyme with effluent and (iii) production of the enzyme dur- 
ing treatment using microorganisms grown on cost-effective substrates. Using immobilized enzymes is a potential solution for industrial-scale application since it enables reusing of biocatalyst and continuous operation and reduces the operational costs (Cabana et al., 2007a). Furthermore, immobilization of enzyme improves the stability of enzyme during storage, against organic solvents and variations in temperature and $\mathrm{pH}$. Immobilization of enzymes also increases the contact surface and helps to avoid too much shear stress which inactivates enzymes (De Cazes et al., 2014a; Majeau et al., 2010; Mateo et al., 2007). However, the immobilized enzyme may have less activity compared to free one due to conformational alterations of the enzyme and its heterogeneity on the support (Davis and Burns, 1992; Fernández-Fernández et al., 2013; Ji et al., 2016b). The immobilization process influences the properties of the biocatalyst and therefore the selection of an immobilization method determines the process specifications of an enzyme such as cost, catalytic activity, effectiveness and deactivation kinetics (Durán et al., 2002; Fernández-Fernández et al., 2013). Also, the toxicity of reagents used for immobilization should be considered for waste disposal and specific application of the biocatalyst (Durán et al., 2002).

In Table 7, the data on the efficiencies of immobilized enzymes for removal of PhACs obtained by different researchers have been summarized. Immobilization of enzymes has been performed on different types of inert (e.g., aluminum oxide pellets and alginate beads) and active (e.g., activated carbon and silica gel) carrier materials. The selection criteria for support materials are being cost-effective and having non-toxic nature, high surface area and mechanical strength (Cabana et al., 2009a; Daâssi et al., 2014). Several immobilization methods have been developed so far i.e. covalent attachment, crosslinking, entrapment in pores or spun fibers, encapsulation, ionic interaction and adsorption (Cabana et al., 2007b, 2009a; Cristóvão et al., 2011; Daâssi et al., 2014).

Immobilization methods that involved chemical reaction with enzyme provided stable attachment and reduced enzyme inactivation rates, however, they tend to reduce the activity of the biocatalyst because the covalent bonds can perturb the native structure of enzyme (Sheldon, 2007). By contrast, physical immobilization and entrapment methods typically have fewer effects on the structure of the enzyme and provide less stability during the reaction (Durán et al., 2002). The appropriate selection of immobilization method depends on several parameters. Normally, an immobilized enzyme with a lower initial activity but long-time stability is preferred to the one with a high initial activity but with less stability (Durán et al., 2002; Sheldon, 2007; Turło and Turło, 2013).

Among these methods, physical adsorption on carriers is a simple and economical approach (Naghdi et al., 2017). To improve the stability and enzyme loading, the carrier surfaces should be properly modified to create functional groups with affinity for protein (Lloret et al., 2012b). Naghdi et al. immobilized Lac on functionalized nanobiochar through acidic treatment and observed an improvement in storage, $\mathrm{pH}$, and thermal stability. The immobilized Lac preserved $70 \%$ of its initial activity after 3 cycles of reaction with ABTS (Naghdi et al., 2017). Similarly, Nguyen et al. immobilized Lac onto functionalized granular activated carbon through acid treatment and observed more than $90 \%$ residual activity after three oxidation cycles using 2, 6-dimethoxy phenol as substrate (Nguyen et al., 2016a). In another study, Ji et al. immobilized Lac on carbon nanotubes via physical adsorption and observed $60 \%$ activity retention after three cycles of ABTS oxidation (Ji et al., 2016a). Therefore, still, physical methods need to be improved since losing $30-40 \%$ of activity in 3 cycles is not economically viable.
Covalently immobilized enzymes showed high performance in removal of PhACs. For example, Kumar et al. used covalently immobilized Lac on electrospun poly(lactic-co-glycolic acid) nanofibers and observed the almost complete removal of DCF in a batch reactor after $5 \mathrm{~h}$. Also, they reported similar degradation efficiency after 3 cycles (Sathishkumar et al., 2012). Likewise, Xu et al. immobilized Lac on polyvinyl alcohol/chitosan nanofibers through covalent bonding for degradation of DCF and observed complete removal after $6 \mathrm{~h}$ in batch mode (Xu et al., 2015b). Also, they employed immobilized HRP on Poly(vinyl alcohol)/poly(acrylic acid) $/ \mathrm{SiO}_{2}$ nanofibers for degradation of paracetamol and obtained more than $80 \%$ removal after $90 \mathrm{~min}$. Around 20\% reduction in degradation efficiency was reported after 3 cycles (Xu et al., 2015a). However, in the mentioned research the initial concentrations of compounds $(>10 \mathrm{ppm})$ were far beyond their environmentally relevant concentration i.e. several $\mathrm{ppb}$.

Cross-linking of enzyme molecules to each other and formation of insoluble enzyme aggregates is another approach to enhance stability. In this method, it is possible to work with or without support. Due to the small size of aggregates, different methods such as microfiltration and using magnetic particles are proposed for separation of the enzyme from effluent (Arca-Ramos et al., 2016b; Kumar and Cabana, 2016). Lac cross-linking enzyme aggregates and Lac-grafted particles have been used for the degradation of PhACs in different reactor configurations e.g. fluidized-bed reactors and fixed-bed reactors (Cao, 2005). For example, Ba et al. used Lac cross-linked aggregates for degradation of the ACT, CBZ, and MFA and observed $99 \%$, nearly $100 \%$ and up to $85 \%$ degradation efficiency respectively after $8 \mathrm{~h}$ in a batch reactor. In continuous experiments, complete removal of ACT and MFA within $24 \mathrm{~h}$ and $93 \%$ removal for CBZ after $72 \mathrm{~h}$ was achieved (Ba et al., 2014b). In another study, a combination of Lac and Tyr were crosslinked to form aggregates and this combined system was used to degrade ACT in municipal wastewaters in batch mode. They observed more than $80 \%-100 \%$ removal for the ACT in municipal wastewater and more than $90 \%$ removal in hospital wastewater (Ba et al., 2014a). Nair et al. immobilized Lac on silica spheres in a two-step adsorption-crosslinking process and achieved more than $30 \%$ of DCF removal in continuous mode (Nair et al., 2013). There are also other research papers that reported different removal efficiency (up to 95\%) while using enzyme aggregates (Kumar and Cabana, 2016; Shi et al., 2014; Touahar et al., 2014).

\section{Enzymatic membrane reactors (EMRs)}

Application of enzymatic treatment in continuous mode still remained a technical challenge since the enzyme is washed out with the treated effluent (Hai et al., 2012). Using membranes with a pore size smaller than the size of enzyme macromolecules is a potential approach to solve this issue (Lloret et al., 2012a). In this, so-called enzymatic membrane reactor (EMR), the enzyme remains in the reactor while continuous feeding and effluent withdrawal are enabled. Compared to the enzyme immobilization approach, EMR has advantages, such as better enzyme dispersion in the reactor, more effective enzyme retention and easier replenishment of fresh enzymes. There are few studies in which continuous PhACs degradation by EMRs is investigated (Asif et al., 2017; Cabana et al., 2009b). Two types of EMRs have been already distinguished (Fig. 3) (Jochems et al., 2011). In the first case (Fig. 3A), which is also called "stirred-tank membrane reactors" the membrane acts as a barrier against escape of free enzyme from the reactor, while transformation products (TPs) are able to cross the membrane along with effluent. In this design, separated devices for reaction and separation are deployed in series and independently controlled in terms of operating conditions (De 
Table 7

Removal efficiencies PhACs by immobilized enzyme in batch experiments.

\begin{tabular}{|c|c|c|c|c|c|c|c|}
\hline Compound & $\begin{array}{l}\text { Initial } \\
\text { PhAC } \\
\text { concentration }\end{array}$ & Source of Fungi & Enzyme & Immobilization method & $\begin{array}{l}\text { Removal } \\
\text { conditions }\end{array}$ & $\begin{array}{l}\text { Removal } \\
(\%)\end{array}$ & References \\
\hline \multirow[t]{4}{*}{ Acetaminophen } & $10-50 \mu \mathrm{g} / \mathrm{L}$ & Trametes versicolor & Laccase & Cross-linked enzyme aggregates & $\begin{array}{l}\text { Wastewater } \\
30{ }^{\circ} \mathrm{C}, 150 \mathrm{rpm}, 6 \mathrm{~h}, \\
10 \mathrm{~mL}\end{array}$ & 26 & $\begin{array}{l}\text { (Arca-Ramos } \\
\text { et al., 2016b) }\end{array}$ \\
\hline & $100 \mu \mathrm{g} / \mathrm{L}$ & Trametes versicolor & Laccase & Cross-linked enzyme aggregates & $\begin{array}{l}\text { Wastewater } \\
20^{\circ} \mathrm{C}, 125 \mathrm{rpm}, 12 \mathrm{~h}, \\
10 \mathrm{~mL}\end{array}$ & 97 & $\begin{array}{l}\text { (Kumar and } \\
\text { Cabana, 2016) }\end{array}$ \\
\hline & $100 \mu \mathrm{g} / \mathrm{L}$ & Trametes versicolor & Laccase & Cross-linked enzyme aggregates & $\begin{array}{l}\text { Wastewater } \\
20^{\circ} \mathrm{C}, 300 \mathrm{rpm}, 120 \mathrm{~h}, \\
3500 \mathrm{~mL}\end{array}$ & 100 & $\begin{array}{l}\text { (Ba et al., } \\
\text { 2014b) }\end{array}$ \\
\hline & $90 \mu \mathrm{g} / \mathrm{L}$ & $\begin{array}{l}\text { Trametes } \\
\text { versicolor/Mushroom }\end{array}$ & Laccase/Tyrosinase & Cross-linked enzyme aggregates & $\begin{array}{l}\text { Wastewater } \\
20^{\circ} \mathrm{C}, 8 \mathrm{~h}\end{array}$ & 93 & $\begin{array}{l}\text { (Ba et al., } \\
\text { 2014a) }\end{array}$ \\
\hline Atenolol & $100 \mu \mathrm{g} / \mathrm{L}$ & Trametes versicolor & Laccase & Cross-linked enzyme aggregates & $\begin{array}{l}\text { Wastewater } \\
20^{\circ} \mathrm{C}, 125 \mathrm{rpm}, 12 \mathrm{~h} \\
10 \mathrm{~mL}\end{array}$ & 90 & $\begin{array}{l}\text { (Kumar and } \\
\text { Cabana, 2016) }\end{array}$ \\
\hline Diazepam & $100 \mu \mathrm{g} / \mathrm{L}$ & Trametes versicolor & Laccase & Cross-linked enzyme aggregates & $\begin{array}{l}\text { Wastewater } \\
20^{\circ} \mathrm{C}, 125 \mathrm{rpm}, 12 \mathrm{~h} \\
10 \mathrm{~mL}\end{array}$ & 68 & $\begin{array}{l}\text { (Kumar and } \\
\text { Cabana, 2016) }\end{array}$ \\
\hline \multirow[t]{7}{*}{ Diclofenac } & $93 \mu \mathrm{g} / \mathrm{L}$ & Trametes versicolor & Laccase & Immobilized on silica nanoparticles & $\begin{array}{l}\text { Wastewater } \\
25^{\circ} \mathrm{C}, 210 \mathrm{rpm}, 24 \mathrm{~h}, \\
100 \mathrm{~mL}\end{array}$ & 0 & $\begin{array}{l}\text { (Arca-Ramos } \\
\text { et al., 2016a) }\end{array}$ \\
\hline & $93 \mu \mathrm{g} / \mathrm{L}$ & $\begin{array}{l}\text { Myceliophthora } \\
\text { thermophila }\end{array}$ & Laccase & Immobilized on silica nanoparticles & $\begin{array}{l}\text { Wastewater } \\
25^{\circ} \mathrm{C}, 210 \mathrm{rpm}, 24 \mathrm{~h}, \\
100 \mathrm{~mL}\end{array}$ & 0 & $\begin{array}{l}\text { (Arca-Ramos } \\
\text { et al., 2016a) }\end{array}$ \\
\hline & $2.5 \mathrm{mg} / \mathrm{L}$ & Aspergillus oryzae & Laccase & $\begin{array}{l}\text { Immobilized on granular activated } \\
\text { carbon }\end{array}$ & $\begin{array}{l}\text { Spiked water } \\
25^{\circ} \mathrm{C}, 70 \mathrm{rpm}, 2 \mathrm{~h} \\
100 \mathrm{~mL}\end{array}$ & 60 & $\begin{array}{l}\text { (Nguyen et al., } \\
\text { 2016a) }\end{array}$ \\
\hline & $50 \mathrm{mg} / \mathrm{L}$ & Pleurotus florida & Laccase & $\begin{array}{l}\text { Immobilized on poly (lactic-co- } \\
\text { glycolic acid) }\end{array}$ & $\begin{array}{l}\text { Spiked water } \\
30^{\circ} \mathrm{C}, 100 \mathrm{rpm}, 5 \mathrm{~h}\end{array}$ & 100 & $\begin{array}{l}\text { (Sathishkumar } \\
\text { et al., 2012) }\end{array}$ \\
\hline & $12.5 \mathrm{mg} / \mathrm{L}$ & $\begin{array}{l}\text { Trametes } \\
\text { versicolor }\end{array}$ & Laccase & $\begin{array}{l}\text { Immobilized on polyvinyl alcohol/ } \\
\text { chitosan/multi-walled } \\
\text { carbon nanotubes }\end{array}$ & $\begin{array}{l}\text { Spiked water } \\
50^{\circ} \mathrm{C}, 6 \mathrm{~h}\end{array}$ & 100 & $\begin{array}{l}\text { (Xu et al., } \\
\text { 2015b) }\end{array}$ \\
\hline & $100 \mu \mathrm{g} / \mathrm{L}$ & Trametes versicolor & Laccase & Cross-linked enzyme aggregates & $\begin{array}{l}\text { Wastewater } \\
20{ }^{\circ} \mathrm{C}, 125 \mathrm{rpm}, 12 \mathrm{~h}, \\
10 \mathrm{~mL}\end{array}$ & 95 & $\begin{array}{l}\text { (Kumar and } \\
\text { Cabana, 2016) }\end{array}$ \\
\hline & $10 \mu \mathrm{M}$ & Coriolopsis gallica & Laccase & $\begin{array}{l}\text { Immobilized on } \\
\text { mesoporous silica spheres }\end{array}$ & $\begin{array}{l}\text { Wastewater } \\
20^{\circ} \mathrm{C}, 24 \mathrm{~h}, 50 \mathrm{~mL}\end{array}$ & 70 & $\begin{array}{l}\text { (Nair et al., } \\
\text { 2013) }\end{array}$ \\
\hline \multirow[t]{4}{*}{ Carbamazepine } & $20 \mu \mathrm{g} / \mathrm{L}$ & Trametes versicolor & Laccase & Immobilized on nanobiochar & $\begin{array}{l}\text { Wastewater } \\
25^{\circ} \mathrm{C}, 200 \mathrm{rpm}, 24 \mathrm{~h}, \\
20 \mathrm{~mL}\end{array}$ & 66 & $\begin{array}{l}\text { (Naghdi et al., } \\
\text { 2017) }\end{array}$ \\
\hline & $5 \mathrm{mg} / \mathrm{L}$ & Trametes versicolor & Laccase & Immobilizedon $\mathrm{TiO}_{2}$ nanoparticles & $\begin{array}{l}\text { Wastewater } \\
25^{\circ} \mathrm{C}, \text { Constant stirring, } \\
96 \mathrm{~h}, 50 \mathrm{~mL}\end{array}$ & 60 & $\begin{array}{c}\text { (Ji et al., } \\
2016 b)\end{array}$ \\
\hline & $2.5 \mathrm{mg} / \mathrm{L}$ & Aspergillus oryzae & Laccase & $\begin{array}{l}\text { Immobilized on granular activated } \\
\text { carbon }\end{array}$ & $\begin{array}{l}\text { Spiked water } \\
25^{\circ} \mathrm{C}, 70 \mathrm{rpm}, 2 \mathrm{~h}, \\
100 \mathrm{~mL}\end{array}$ & 40 & $\begin{array}{l}\text { (Nguyen et al., } \\
\text { 2016a) }\end{array}$ \\
\hline & $100 \mu \mathrm{g} / \mathrm{L}$ & Trametes versicolor & Laccase & Cross-linked enzyme aggregates & $\begin{array}{l}\text { Wastewater } \\
20^{\circ} \mathrm{C}, 300 \mathrm{rpm}, 120 \mathrm{~h} \\
3500 \mathrm{~mL}\end{array}$ & 18 & $\begin{array}{l}\text { (Ba et al., } \\
2014 b)\end{array}$ \\
\hline Chlortetracycline & $200 \mu \mathrm{g} / \mathrm{L}$ & Trametes versicolor & Laccase & Immobilized on nanofibers & $\begin{array}{l}\text { Spiked water } \\
25^{\circ} \mathrm{C} \text {, continuous mode } \\
\left(1 \mathrm{~mL} / \mathrm{h} \cdot \mathrm{cm}^{2}\right)\end{array}$ & 58.3 & $\begin{array}{l}\text { (Taheran et al., } \\
\text { 2017b) }\end{array}$ \\
\hline \multirow[t]{2}{*}{ Fenofibrate } & $10-50 \mu \mathrm{g} / \mathrm{L}$ & Trametes versicolor & Laccase & Cross-linked enzyme aggregates & $\begin{array}{l}\text { Wastewater } \\
30^{\circ} \mathrm{C}, 150 \mathrm{rpm}, 6 \mathrm{~h}, \\
10 \mathrm{~mL}\end{array}$ & 37 & $\begin{array}{l}\text { (Arca-Ramos } \\
\text { et al., 2016b) }\end{array}$ \\
\hline & $100 \mu \mathrm{g} / \mathrm{L}$ & Trametes versicolor & Laccase & Cross-linked enzyme aggregates & $\begin{array}{l}\text { Wastewater } \\
20^{\circ} \mathrm{C}, 125 \mathrm{rpm}, 12 \mathrm{~h}, \\
10 \mathrm{~mL}\end{array}$ & 45 & $\begin{array}{l}\text { (Kumar and } \\
\text { Cabana, 2016) }\end{array}$ \\
\hline Ketoprofen & $100 \mu \mathrm{g} / \mathrm{L}$ & Trametes versicolor & Laccase & Cross-linked enzyme aggregates & $\begin{array}{l}\text { Wastewater } \\
20{ }^{\circ} \mathrm{C}, 125 \mathrm{rpm}, 12 \mathrm{~h}, \\
10 \mathrm{~mL}\end{array}$ & 48 & $\begin{array}{l}\text { (Kumar and } \\
\text { Cabana, 2016) }\end{array}$ \\
\hline \multirow[t]{2}{*}{ Mefenamic acid } & $100 \mu \mathrm{g} / \mathrm{L}$ & Trametes versicolor & Laccase & Cross-linked enzyme aggregates & $\begin{array}{l}\text { Wastewater } \\
20^{\circ} \mathrm{C}, 125 \mathrm{rpm}, 12 \mathrm{~h}, \\
10 \mathrm{~mL}\end{array}$ & 99 & $\begin{array}{l}\text { (Kumar and } \\
\text { Cabana, 2016) }\end{array}$ \\
\hline & $100 \mu \mathrm{g} / \mathrm{L}$ & Trametes versicolor & Laccase & Cross-linked enzyme aggregates & $\begin{array}{l}\text { Wastewater } \\
20^{\circ} \mathrm{C}, 300 \mathrm{rpm}, 120 \mathrm{~h} \text {, } \\
3500 \mathrm{~mL}\end{array}$ & 100 & $\begin{array}{l}\text { (Ba et al., } \\
\text { 2014b) }\end{array}$ \\
\hline Paracetamol & $20 \mathrm{mg} / \mathrm{L}$ & Not mentioned & $\begin{array}{c}\text { Horseradish } \\
\text { peroxidase }\end{array}$ & $\begin{array}{l}\text { Immobilized on nanofibrous } \\
\text { membranes }\end{array}$ & $\begin{array}{l}\text { Spiked water } \\
25^{\circ} \mathrm{C}, 90 \mathrm{~min}, 50 \mathrm{~mL}\end{array}$ & 98 & $\begin{array}{l}\text { (Xu et al., } \\
\text { 2015a) }\end{array}$ \\
\hline Sulfadiazine & $50 \mathrm{mg} / \mathrm{L}$ & Echinodontium taxodii & Laccase & Immobilized on $\mathrm{Fe}_{3} \mathrm{O}_{4}$ nanoparticles & $\begin{array}{l}\text { Spiked water } \\
25^{\circ} \mathrm{C}, 5 \mathrm{~min}\end{array}$ & 100 & $\begin{array}{l}\text { (Shi et al., } \\
\text { 2014) }\end{array}$ \\
\hline
\end{tabular}


Table 7 (Continued)

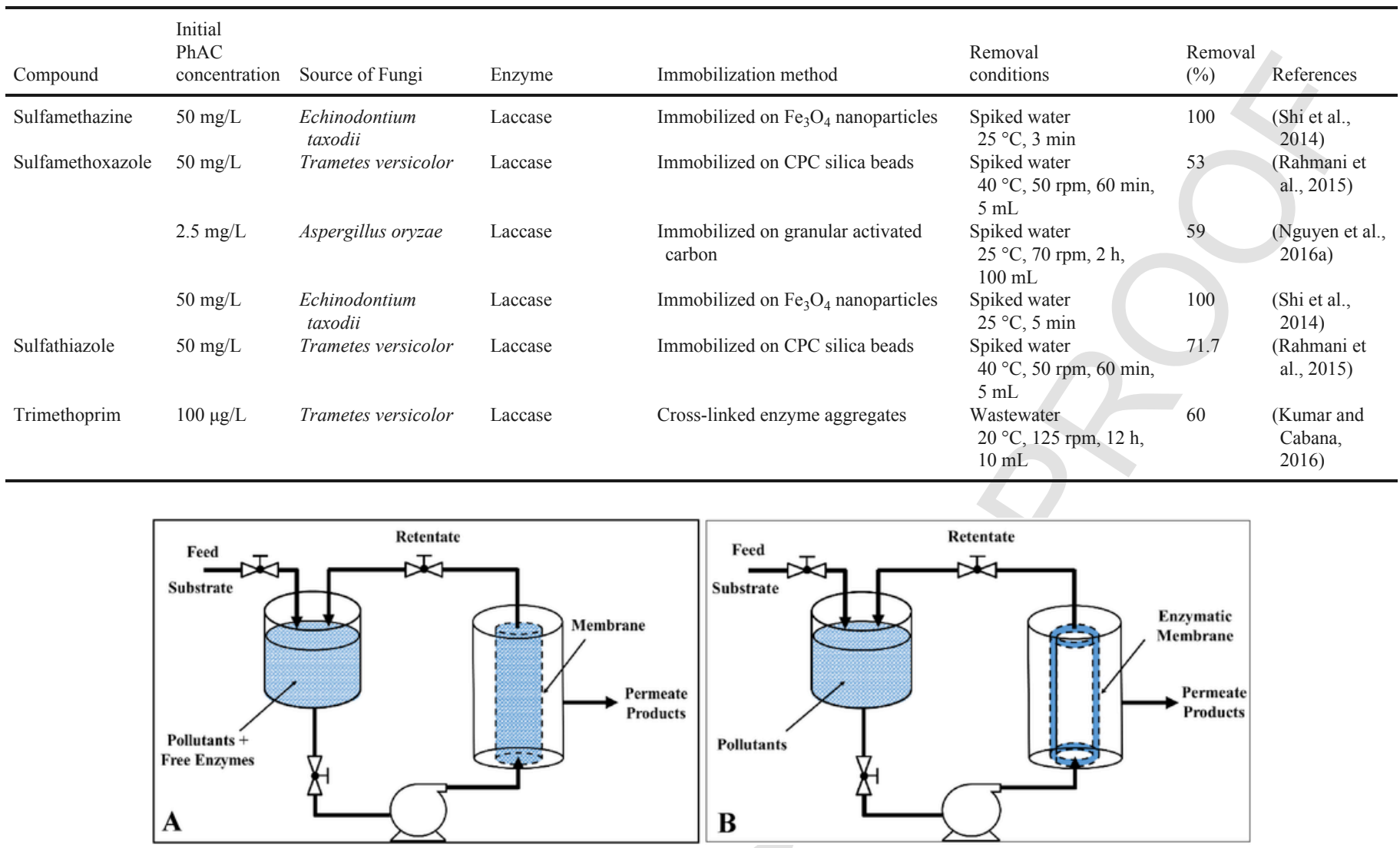

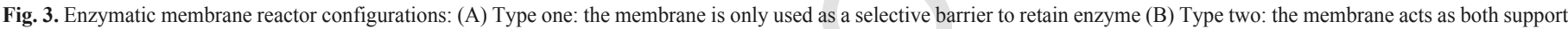
for biocatalyst and selective barrier.

Cazes et al., 2014a). One of the challenges for this type of EMR is adjusting the tangential flow to avoid concentration polarization, to prevent enzyme inactivation and to achieve a feasible filtration rate. Stirred-tank membrane reactors have been already studied for hydrolysis reactions, but such reactors were also studied for environmental applications. Recent investigations have focused on the removal of DCF, CBZ, and SMX from water and wastewater. For instance, Nguyen et al. studied the effect of the addition of granular activated carbon (GAC) to Lac-based stirred-tank membrane reactors on the removal of CBZ, DCF, and SMX. They observed that dosing of $3 \mathrm{~g} / \mathrm{L}$ GAC caused 14-25\% improvement in biodegradation of the PhACs and reduced membrane fouling. They also found that the removal of DCF increased from $30 \%$ in a batch reactor ( $8 \mathrm{~h}$ contact time) to $60 \%$ in EMR continuous operation ( $8 \mathrm{~h}$ retention time) (Nguyen et al., $2014 \mathrm{c}$ ). In a related study, they observed that addition of SA as a mediator at $5 \mu \mathrm{M}$ can increase the removal efficiency from $60 \%$ to $80 \%$ under DCF loading rate of $480 \mu \mathrm{g} / \mathrm{L}$.d. They also found that DCF was trapped into the gel layer of enzyme formed on the membrane surface, which helped in its biodegradation (Nguyen et al., 2014b, 2015). They also found that a higher concentration of SA increased the toxicity of effluent, due to the presence of generated radicals and unconsumed SA (Nguyen et al., 2016b). A gradual depletion of the enzymatic activity has been reported even with properly selected molecular weight cut-off, which indicates enzyme denaturation during operation. Depletion of enzymatic activity is caused by both natural activity decay of free enzymes and inactivation due to shear stresses. Therefore, the periodic addition of enzyme to the reactor will be required to maintain the level of enzymatic activity (Nguyen et al., 2014b, 2014c).
In the second type of EMRs (Fig. 3B), the membrane is a selective barrier, which was already used as a support for immobilization of enzyme. Therefore, the biocatalytic reaction happens at many places where the enzyme is immobilized i.e. the external/internal surface of the membrane. This configuration offers several advantages, such as lower energy consumption compared to packed bed reactors, enzyme stability and reducing the blockage of the membrane and forcing the pollutants to pass over the active sites during filtration. The latter is considered as the main benefit of this process (Sanchez Marcano and Tsotsis, 2004). In this type of EMRs, the collision between enzyme and substrate occurs during the mass transfer process through the membrane. Therefore, the biocatalytic reaction takes place during the mass transfer process and the TPs are released into the permeate. This concept enables better control of the process by reducing the distance between the substrate and catalyst and increasing the probability of reaction. The membrane is an assembly of pores, which can be considered as micro-reactors. In these micro-reactors, the contact between reactants is improved since the path for mass transfer is reduced and simultaneously the retention time can be adjusted by manipulating the flux rate (Jochems et al., 2011; Rios et al., 2004).

The selection of immobilization method is based on membrane properties, enzyme properties, and cost. There are three main techniques for preparation of active membranes: attachment through covalent or physical bonds on the membrane, entrapment in the pores and deposition of a gel layer of enzymes on the surface of the membrane (Hilal et al., 2004; Kanwar and Goswami, 2002).

Attachment through covalent bonding methods such as the formation of carbodiimides, diazonium salts, etc. is advantageous in terms 
of enzyme stability and leaching prevention. De Cazes et al. covalently immobilized Lac onto the ceramic membrane and degraded TC at $56 \%$ efficiency after $24 \mathrm{~h}$ whereas the efficiency was only $30 \%$ with free Lac. Furthermore, their EMR reached a constant degradation rate during 10 days (De Cazes et al., 2014b; de Cazes et al., 2015). However covalent bonding to support is not a preferred method due to reduction of activity, high cost and regeneration problems (Belleville et al., 2001; Chea et al., 2012; Durante et al., 2004; Hou et al., 2014; Mateo et al., 2007; Xu et al., 2006). On the other hand, entrapping enzyme in the pores and formation of gel on the surface of membrane are simple, cheap and offer the possibility of regeneration and their leaching can be overcome by forming enzymes clusters inside the membrane pores (Paiva et al., 2000; Sakaki et al., 2001; Trusek-Holownia and Noworyta, 2007). Also, the stability of the enzyme layer can be improved by covalent bonding of enzyme molecules to each other (Yujun et al., 2008). It is obvious that further studies are needed to advance in EMR design, particularly to verify their viability at large scales and their potential challenges such as fouling. A mathematical study by Abejon et al. on immobilized Lac for degradation of antibiotics showed that this process is still far from economic competitiveness due to the costs of membrane conditioning. They concluded that some improvements on the lifetime of the reactors, enzymatic activity, and membrane conditioning or regeneration costs need to be made to achieve competitive economical (Abejón et al., 2015).

\section{Hybrid methods}

The combination and enzymatic degradation with other treatment methods attracted the attention of researchers due to the potential of overcoming the drawbacks of single process approaches. For example, placing fungal reactor and activated sludge system in series can combine the benefits of both systems, namely the oxidative activities with fungi and decreasing chemical oxygen demand (COD) by activated sludge (Anastasi et al., 2012). Also, a combination of sonication with oxidation by Lac enzyme improves the degradation efficiency and reduces reaction time (Sutar and Rathod, 2015a, 2016). Ultrasonication produces radicals that independently attack and oxidize the contaminant molecules (Sutar and Rathod, 2015b). Combination of fungal media with Fenton system is another strategy studied for degradation of PhACs. In this system, the degradation efficiency of recalcitrant $\mathrm{CBZ}$ was reported to reach $80 \%$ after addition of 2,6-dimethoxy-1,4-benzoquinone and $\mathrm{Fe}^{3+}$-oxalate to fungal culture (Marco-Urrea et al., 2010d). Yang studied the augmentation of an MBR with TV to take the advantage of both fungi and bacteria for removal of DCF. Initially, they observed the high removal of DCF, which gradually dropped from $80 \%$ to $40 \%$ after three weeks of operation in continuous mode. Their mass balance showed that $66 \%$ of adsorbed DCF onto sludge underwent biodegradation that reveals a successful combination. However, the loss of extracellular laccase through membrane should be mentioned as a drawback (Yang, 2012).

\section{Conclusion and future outlook}

Over the past 20 years, numerous researchers have investigated the performance of different processes to remove PhACs from water and wastewaters. Treatment systems based on WRFs and their related oxidoreductase enzyme systems offer a promising and environmentally friendly solution for removing such pollutants. This method has advantages over other treatment methods, such as the production of less toxic by-products and producing no concentrated stream.
The reviewed literature showed that a variety of pharmaceuticals are efficiently removed by both crude/purified enzymes and whole-cell fungi. Treatment with whole-cell fungi showed superior performance for many compounds due to the synergistic effects of intracellular and extracellular enzymes coupled with sorption onto fungal biomass. However, in these systems, the washing out of enzymes with effluent and constant supply of different nutrients to keep fungi active increase the organic loading of final effluent. Furthermore, the bacterial contamination of fungal culture should be considered for large-scale applications since in real water and wastewater, there are different consortia of microorganisms that can compete with fungal activity. Also, there are factors including temperature, $\mathrm{pH}$, aeration, and dissolved constituents that affect the removal performance in enzymatic treatment system and this needs to be investigated. Based on the data obtained from the effects of different factors, the limitations of enzymatic treatment in terms of influent characteristics, operational conditions and effluent quality will be elucidated. Performing the enzymatic treatment in pilot scale is needed $f$ the operational problems and to estimate the capital and operational costs for large-scale applications.

While using extracted enzymes, the lower stability of enzyme and its loss with the effluent urged researchers to insolubilize or immobilize the free enzyme through different methods. Although immobilization can increase the stability and enable reusability, most of the immobilization methods have significant drawbacks, such as being highly expensive, loss of enzyme activity and regeneration problems. Low molecular weight redox mediators can enhance the kinetic and degradation efficiency of enzymes, however, they deplete the enzyme activity and may pose toxicity to the final effluents.

Although ligninolytic enzymes and especially laccases were already commercialized for applications, such as denim bleaching, still there are significant hurdles in the commercialization of these enzymes for waste stream bioremediation. Large amounts of required enzyme, the high cost of mediators, production of toxic compounds and losing enzyme activity as a result of inhibitors in the waste stream are among the most important hurdles that need to be addressed in research phase before proceeding to commercialization phase.

According to the performed studies on by-products of enzymatic treatment of PhACs till date, final TPs are less toxic as compared to the parent compounds in many cases. However, there are several examples of enzymatic treatment by-products, such as hydroxylated IBP, which is more toxic compared to IBP. Therefore, the major TPs of enzymatic treatment can be identified for the majority of present compounds in the waste streams and their toxicity should be determined through standard methods prior to the decision for the system scale-up.

Recent investigations suggested using immobilized enzymes in hybrid processes to improve pollutant degradation. These complementary processes, such as adsorption, Fenton oxidation, and ultrasonication can improve the degradation of recalcitrant compounds, such as CBZ. Further investigation is required to evaluate the technical, economical and environmental aspects of different process combinations to obtain a reliable and robust strategy for degradation of micropollutants.

\section{Acknowledgements}

The authors are sincerely thankful to the Natural Sciences and Engineering Research Council of Canada (Discovery Grant 355254 and Strategic Grants), and Ministère des Relations Internationales du Québec (122523) (coopération Québec-Catalanya 2012-2014) for fi- 
nancial support. INRS-ETE is thanked for providing Mr. Mehrdad Taheran "Bourse d'excellence" scholarship for his Ph.D. studies. The views or opinions expressed in this article are those of the authors.

\section{References}

Abejón, R., Belleville, M.P., Sanchez-Marcano, J., 2015. Design, economic evaluation and optimization of enzymatic membrane reactors for antibiotics degradation in wastewaters. Sep. Purif. Technol. 156, 183-199, Part 2.

Alexander, J.T., Hai, F.I., Al-aboud, T.M., 2012. Chemical coagulation-based processes for trace organic contaminant removal: current state and future potential. J. Environ. Manag. 111, 195-207.

Anastasi, A., Spina, F., Romagnolo, A., Tigini, V., Prigione, V., Varese, G.C., 2012. Integrated fungal biomass and activated sludge treatment for textile wastewaters bioremediation. Bioresour. Technol. 123, 106-111.

Arca-Ramos, A., Ammann, E., Gasser, C., Nastold, P., Eibes, G., Feijoo, G., Lema, J., Moreira, M., Corvini, P.-X., 2016a. Assessing the use of nanoimmobilized laccases to remove micropollutants from wastewater. Environ. Sci. Pollut. Res. 23, $3217-3228$.

Arca-Ramos, A., Kumar, V., Eibes, G., Moreira, M., Cabana, H., 2016b. Recyclable cross-linked laccase aggregates coupled to magnetic silica microbeads for elimination of pharmaceuticals from municipal wastewater. Environ. Sci. Pollut. Res. 23, 8929-8939.

Arora, D.S., Sharma, R.K., 2010. Ligninolytic fungal laccases and their biotechnological applications. Appl. Biochem. Biotechnol. 160, 1760-1788.

Asgher, M., Asad, M.J., Bhatti, H.N., Legge, R.L., 2007. Hyperactivation and thermostabilization of Phanerochaete chrysosporium lignin peroxidase by immobilization in xerogels. World J. Microbiol. Biotechnol. 23, 525-531.

Asgher, M., Bhatti, H.N., Ashraf, M., Legge, R.L., 2008. Recent developments in biodegradation of industrial pollutants by white rot fungi and their enzyme system. Biodegradation 19, 771 .

Ashe, B., Nguyen, L.N., Hai, F.I., Lee, D.-J., van de Merwe, J.P., Leusch, F.D., Price, W.E., Nghiem, L.D., 2016. Impacts of redox-mediator type on trace organic contaminants degradation by laccase: degradation efficiency, laccase stability and effluent toxicity. Int. Biodeterior. Biodegrad. 113, 169-176.

Asif, M.B., Hai, F.I., Singh, L., Price, W.E., Nghiem, L.D., 2017. Degradation of pharmaceuticals and personal care products by white-rot fungi-a critical review. Curr. Pollut. Rep. 1-16.

Astolfi, P., Brandi, P., Galli, C., Gentili, P., Gerini, M.F., Greci, L., Lanzalunga, O., 2005. New mediators for the enzyme laccase: mechanistic features and selectivity in the oxidation of non-phenolic substrates. New J. Chem. 29, 1308-1317.

Ba, S., Haroune, L., Cruz-Morató, C., Jacquet, C., Touahar, I.E., Bellenger, J.-P., Legault, C.Y., Jones, J.P., Cabana, H., 2014a. Synthesis and characterization of combined cross-linked laccase and tyrosinase aggregates transforming acetaminophen as a model phenolic compound in wastewaters. Sci. Total Environ. 487, $748-755$.

Ba, S., Jones, J.P., Cabana, H., 2014b. Hybrid bioreactor (HBR) of hollow fiber microfilter membrane and cross-linked laccase aggregates eliminate aromatic pharmaceuticals in wastewaters. J. Hazard. Mater. 280, 662-670.

Baborová, P., Möder, M., Baldrian, P., Cajthamlová, K., Cajthaml, T., 2006. Purification of a new manganese peroxidase of the white-rot fungus Irpex lacteus, and degradation of polycyclic aromatic hydrocarbons by the enzyme. Res. Microbiol. $157,248-253$.

Baldrian, P., 2006. Fungal laccases-occurrence and properties. FEMS Microbiol. Rev. $30,215-242$.

Bathen, D., 2003. Physical waves in adsorption technology-an overview. Sep. Purif. Technol. 33, 163-177.

Bauer, C.G., Kühn, A., Gajovic, N., Skorobogatko, O., Holt, P.-J., Bruce, N.C., Makower, A., Lowe, C.R., Scheller, F.W., 1999. New enzyme sensors for morphine and codeine based on morphine dehydrogenase and laccase. Fresenius' J. Anal. Chem. 364, 179-183.

Becker, D., Della Giustina, S.V., Rodriguez-Mozaz, S., Schoevaart, R., Barceló, D., de Cazes, M., Belleville, M.-P., Sanchez-Marcano, J., de Gunzburg, J., Couillerot, O., 2016. Removal of antibiotics in wastewater by enzymatic treatment with fungal laccase-Degradation of compounds does not always eliminate toxicity. Bioresour. Technol. 219, 500-509.

Belleville, M., Lozano, P., Iborra, J., Rios, G., 2001. Preparation of hybrid membranes for enzymatic reaction. Sep. Purif. Technol. 25, 229-233.

Bento, I., Martins, L.O., Lopes, G.G., Carrondo, M.A., Lindley, P.F., 2005. Dioxygen reduction by multi-copper oxidases; a structural perspective. Dalton Trans. 3507-3513.

Bistan, M., Podgorelec, M., Marinšek Logar, R., Tišler, T., 2012. Yeast estrogen screen assay as a tool for detecting estrogenic activity in water bodies. Food Technol. Biotechnol. 50, 427-433.

Blanford, C.F., Foster, C.E., Heath, R.S., Armstrong, F.A., 2009. Efficient electrocatalytic oxygen reduction by the 'blue'copper oxidase, laccase, directly attached to chemically modified carbons. Faraday Discuss. 140, 319-335.
Bosco, F., Capolongo, A., Ruggeri, B., 2002. Effect of temperature, pH, ionic strength, and sodium nitrate on activity of LiPs: implications for bioremediation. Bioremediation J. 6, 65-76.

Buchicchio, A., Bianco, G., Sofo, A., Masi, S., Caniani, D., 2016. Biodegradation of carbamazepine and clarithromycin by Trichoderma harzianum and Pleurotus ostreatus investigated by liquid chromatography - high-resolution tandem mass spectrometry (FTICR MS-IRMPD). Sci. Total Environ. 557, 733-739.

Bumpus, J., Tien, M., Wright, D., Aust, S., 1985. Oxidation of persistent environmental pollutants by a white rot fungus. Science 228, 1434-1436.

Burkhardt-Holm, P., 2011. Linking water quality to human health and environment: the fate of micropollutants. Inst. Water Policy Natl. Univ. Singap. 1-62.

Cabana, H., Jones, J., Agathos, S., 2007a. Elimination of endocrine disrupting chemicals using white rot fungi and their lignin modifying enzymes: a review. Eng. Life Sci. 7, 429-456.

Cabana, H., Jones, J.P., Agathos, S.N., 2007b. Preparation and characterization of cross-linked laccase aggregates and their application to the elimination of endocrine disrupting chemicals. J. Biotechnol. 132, 23-31.

Cabana, H., Alexandre, C., Agathos, S.N., Jones, J.P., 2009a. Immobilization of laccase from the white rot fungus Coriolopsis polyzona and use of the immobilized biocatalyst for the continuous elimination of endocrine disrupting chemicals. Bioresour. Technol. 100, 3447-3458.

Cabana, H., Jones, J.P., Agathos, S.N., 2009b. Utilization of cross-linked laccase aggregates in a perfusion basket reactor for the continuous elimination of endocrine-disrupting chemicals. Biotechnol. Bioeng. 102, 1582-1592.

Camarero, S., Sarkar, S., Ruiz-Dueñas, F.J., Martinez, M.a.J., Martínez, Á.T., 1999. Description of a versatile peroxidase involved in the natural degradation of lignin that has both manganese peroxidase and lignin peroxidase substrate interaction sites. J. Biol. Chem. 274, 10324-10330.

Cao, L., 2005. Immobilised enzymes: science or art?. Curr. Opin. Chem. Biol. 9, 217-226.

Carvalho, A., Fonseca, T., Mattos, M., Oliveira, M., Lemos, T., Molinari, F., Romano, D., Serra, I., 2015. Recent advances in lipase-mediated preparation of pharmaceuticals and their intermediates. Int. J. Mol. Sci. 16, 26191.

Chea, V., Paolucci-Jeanjean, D., Belleville, M., Sanchez, J., 2012. Optimization and characterization of an enzymatic membrane for the degradation of phenolic compounds. Catal. Today 193, 49-56.

Cheng, X.-B., Rong, J., Ping-Sheng, L., Qin, Z., Shi-Qian, T., Wen-Zhong, T., 2007. Studies on the properties and co-immobilization of manganese peroxidase. Chin. J. Biotechnol. 23, 90-96.

Christian, V., Shrivastava, R., Shukla, D., Modi, H., Vyas, B., 2005a. Degradation of Xenobiotic Compounds by Lignin-degrading White-rot Fungi: Enzymology and Mechanisms Involved.

Christian, V., Shrivastava, R., Shukla, D., Modi, H., Vyas, B.R.M., 2005b. Mediator role of veratryl alcohol in the lignin peroxidase-catalyzed oxidative decolorization of remazol brilliant blue R. Enzyme Microb. Technol. 36, 327-332.

Christian, V., Shrivastava, R., Shukla, D., Modi, H.A., Vyas, B.R.M., 2005c. Degradation of xenobiotic compounds by lignin-degrading white-rot fungi: enzymology and mechanisms involved. Indian J. Exp. Biol. 43, 301-312.

Coniglio, A., Galli, C., Gentili, P., Vadala, R., 2008. Oxidation of amides by laccase-generated aminoxyl radicals. J. Mol. Catal. B Enzym. 50, 40-49.

Cozzolino, A., Piccolo, A., 2002. Polymerization of dissolved humic substances catalyzed by peroxidase. Effects of $\mathrm{pH}$ and humic composition. Org. Geochem. 33, 281-294.

Cristóvão, R.O., Tavares, A.P., Brígida, A.I., Loureiro, J.M., Boaventura, R.A., Macedo, E.A., Coelho, M.A.Z., 2011. Immobilization of commercial laccase onto green coconut fiber by adsorption and its application for reactive textile dyes degradation. J. Mol. Catal. B Enzym. 72, 6-12.

Cruz-Morató, C., Rodríguez-Rodríguez, C., Marco-Urrea, E., Sarrà, M., Caminal, G., Vicent, T., Jelić, A., García-Galán, M., Pérez, S., Díaz-Cruz, M., 2012. Biodegradation of Pharmaceuticals by Fungi and Metabolites Identification, Emerging Organic Contaminants in Sludges. Springer, 165-213.

Cruz-Morató, C., Ferrando-Climent, L., Rodriguez-Mozaz, S., Barceló, D., Marco-Urrea, E., Vicent, T., Sarrà, M., 2013. Degradation of pharmaceuticals in non-sterile urban wastewater by Trametes versicolor in a fluidized bed bioreactor. Water Res. $47,5200-5210$.

Cruz-Morató, C., Lucas, D., Llorca, M., Rodriguez-Mozaz, S., Gorga, M., Petrovic, M., Barceló, D., Vicent, T., Sarrà, M., Marco-Urrea, E., 2014. Hospital wastewater treatment by fungal bioreactor: removal efficiency for pharmaceuticals and endocrine disruptor compounds. Sci. Total Environ. 493, 365-376.

Daâssi, D., Rodríguez-Couto, S., Nasri, M., Mechichi, T., 2014. Biodegradation of textile dyes by immobilized laccase from Coriolopsis gallica into $\mathrm{Ca}$-alginate beads. Int. Biodeterior. Biodegrad. 90, 71-78.

Davis, S., Burns, R.G., 1992. Covalent immobilization of laccase on activated carbon for phenolic effluent treatment. Appl. Microbiol. Biotechnol. 37, 474-479.

De Cazes, M., Abejón, R., Belleville, M.-P., Sanchez-Marcano, J., 2014a. Membrane bioprocesses for pharmaceutical micropollutant removal from waters. Membranes 4, 692-729.

De Cazes, M., Belleville, M.-P., Petit, E., Llorca, M., Rodríguez-Mozaz, S., De Gunzburg, J., Barceló, D., Sanchez-Marcano, J., 2014b. Design and optimization of an 
enzymatic membrane reactor for tetracycline degradation. Catal. Today 236, $146-152$.

de Cazes, M.d., Belleville, M.-P., Mougel, M., Kellner, H., Sanchez-Marcano, J., 2015 Characterization of laccase-grafted ceramic membranes for pharmaceuticals degradation. J. Membr. Sci. 476, 384-393.

De García, S.O., Pinto, G.P., García-Encina, P.A., Mata, R.I., 2013. Ranking of concern, based on environmental indexes, for pharmaceutical and personal care products: an application to the Spanish case. J. Environ. Manag. 129, 384-397.

Deblonde, T., Cossu-Leguille, C., Hartemann, P., 2011. Emerging pollutants in wastewater: a review of the literature. Int. J. Hyg. Environ. health 214, 442-448.

Demarche, P., Junghanns, C., Nair, R.R., Agathos, S.N., 2012. Harnessing the power of enzymes for environmental stewardship. Biotechnol. Adv. 30, 933-953.

Duran, N., Esposito, E., 2000. Potential applications of oxidative enzymes and phenoloxidase-like compounds in wastewater and soil treatment: a review. Appl. Catal. B Environ. 28, 83-99.

Durán, N., Rosa, M.A., D'Annibale, A., Gianfreda, L., 2002. Applications of laccases and tyrosinases (phenoloxidases) immobilized on different supports: a review. Enzyme Microb. Technol. 31, 907-931.

Durante, D., Casadio, R., Martelli, L., Tasco, G., Portaccio, M., De Luca, P., Bencivenga, U., Rossi, S., Di Martino, S., Grano, V., 2004. Isothermal and non-isothermal bioreactors in the detoxification of waste waters polluted by aromatic compounds by means of immobilised laccase from Rhus vernicifera. J. Mol. Catal. B Enzym. 27, 191-206.

D'Acunzo, F., Galli, C., Gentili, P., Sergi, F., 2006. Mechanistic and steric issues in the oxidation of phenolic and non-phenolic compounds by laccase or laccase-mediator systems. The case of bifunctional substrates. New J. Chem. 30, 583-591.

D'Souza-Ticlo, D., Sharma, D., Raghukumar, C., 2009. A thermostable metal-tolerant laccase with bioremediation potential from a marine-derived fungus. Mar. Biotechnol. 11, 725-737.

Eibes, G., Debernardi, G., Feijoo, G., Moreira, M.T., Lema, J.M., 2011. Oxidation of pharmaceutically active compounds by a ligninolytic fungal peroxidase. Biodegradation 22, 539-550.

Espinosa-Ortiz, E.J., Rene, E.R., Pakshirajan, K., van Hullebusch, E.D., Lens, P.N., 2016. Fungal pelleted reactors in wastewater treatment: applications and perspectives. Chem. Eng. J. 283, 553-571.

Fabbrini, M., Galli, C., Gentili, P., 2002. Comparing the catalytic efficiency of some mediators of laccase. J. Mol. Catal. B Enzym. 16, 231-240.

Fernández-Fernández, M., Sanromán, , Moldes, D., 2013. Recent developments and applications of immobilized laccase. Biotechnol. Adv. 31, 1808-1825.

Ferrando-Climent, L., Cruz-Morató, C., Marco-Urrea, E., Vicent, T., Sarrà, M., Rodriguez-Mozaz, S., Barceló, D., 2015. Non conventional biological treatment based on Trametes versicolor for the elimination of recalcitrant anticancer drugs in hospital wastewater. Chemosphere 136, 9-19.

Gao, D., Zeng, Y., Wen, X., Qian, Y., 2008. Competition strategies for the incubation of white rot fungi under non-sterile conditions. Process Biochem. 43, 937-944.

García-Galán, M.J., Rodríguez-Rodríguez, C.E., Vicent, T., Caminal, G., Díaz-Cruz, M.S., Barceló, D., 2011. Biodegradation of sulfamethazine by Trametes versicolor: removal from sewage sludge and identification of intermediate products by UPLC-QqTOF-MS. Sci. Total Environ. 409, 5505-5512.

Garcia-Ruiz, E., Mate, D.M., Gonzalez-Perez, D., Molina-Espeja, P., Camarero, S., Martínez, A.T., Ballesteros, A.O., Alcalde, M., 2014. Directed evolution of ligninolytic oxidoreductases: from functional expression to stabilization and beyond. Cascade Biocatal. 1-22.

Gianfreda, L., Sannino, F., Filazzola, M., Leonowicz, A., 1998. Catalytic behavior and detoxifying ability of a laccase from the fungal strain Cerrena unicolor. J. Mol. Catal. B Enzym. 4, 13-23.

Golan-Rozen, N., Chefetz, B., Ben-Ari, J., Geva, J., Hadar, Y., 2011. Transformation of the recalcitrant pharmaceutical compound carbamazepine by Pleurotus ostreatus: role of cytochrome P450 monooxygenase and manganese peroxidase. Environ. Sci. Technol. 45, 6800-6805.

Golan-Rozen, N., Seiwert, B., Riemenschneider, C., Reemtsma, T., Chefetz, B., Hadar, Y., 2015. Transformation pathways of the recalcitrant pharmaceutical compound carbamazepine by the white-rot fungus Pleurotus ostreatus: effects of growth conditions. Environ. Sci. Technol. 49, 12351-12362.

Gold, M.H., Wariishi, H., Valli, K., 1989. Extracellular Peroxidases Involved in Lignin Degradation by the White Rot Basidiomycete Phanerochaete Chrysosporium. ACS Publications.

González, L.F., Sarria, V., Sánchez, O.F., 2010. Degradation of chlorophenols by sequential biological-advanced oxidative process using Trametes pubescens and $\mathrm{TiO}$ 2/UV. Bioresour. Technol. 101, 3493-3499.

Grandclément, C., Seyssiecq, I., Piram, A., Wong-Wah-Chung, P., Vanot, G., Tiliacos N., Roche, N., Doumenq, P., 2017. From the conventional biological wastewater treatment to hybrid processes, the evaluation of organic micropollutant removal: a review. Water Res.

Gros, M., Cruz-Morato, C., Marco-Urrea, E., Longrée, P., Singer, H., Sarrà, M., Hollender, J., Vicent, T., Rodriguez-Mozaz, S., Barceló, D., 2014. Biodegradation of the X-ray contrast agent iopromide and the fluoroquinolone antibiotic ofloxacin by the white rot fungus Trametes versicolor in hospital wastewaters and identification of degradation products. Water Res. 60, 228-241.
Guo, X.-1., Zhu, Z.-w., Li, H.-1., 2014. Biodegradation of sulfamethoxazole by Phanerochaete chrysosporium. J. Mol. Liq. 198, 169-172.

Hai, F.I., Yamamoto, K., Nakajima, F., Fukushi, K., 2009. Factors governing performance of continuous fungal reactor during non-sterile operation-the case of a membrane bioreactor treating textile wastewater. Chemosphere 74, 810-817.

Hai, F.I., Yamamoto, K., Nakajima, F., Fukushi, K., 2012. Application of a GAC-coated hollow fiber module to couple enzymatic degradation of dye on membrane to whole cell biodegradation within a membrane bioreactor. J. Membr. Sci. 389, 67-75.

Hata, T., Kawai, S., Okamura, H., Nishida, T., 2010a. Removal of diclofenac and mefenamic acid by the white rot fungus Phanerochaete sordida YK-624 and identification of their metabolites after fungal transformation. Biodegradation 21, 681-689.

Hata, T., Shintate, H., Kawai, S., Okamura, H., Nishida, T., 2010b. Elimination of carbamazepine by repeated treatment with laccase in the presence of 1-hydroxybenzotriazole. J. Hazard. Mater. 181, 1175-1178.

Hatakka, A., 1994. Lignin-modifying enzymes from selected white-rot fungi: production and role from in lignin degradation. FEMS Microbiol. Rev. 13, 125-135.

Hilal, N., Nigmatullin, R., Alpatova, A., 2004. Immobilization of cross-linked lipase aggregates within microporous polymeric membranes. J. Membr. Sci. 238, 131-141.

Hirai, H., Sugiura, M., Kawai, S., Nishida, T., 2005. Characteristics of novel lignin peroxidases produced by white-rot fungus Phanerochaete sordida YK-624. FEMS Microbiol. Lett. 246, 19-24.

Hou, J., Dong, G., Ye, Y., Chen, V., 2014. Laccase immobilization on titania nanoparticles and titania-functionalized membranes. J. Membr. Sci. 452, 229-240.

Hu, X., Wang, C., Wang, L., Zhang, R., Chen, H., 2014. Influence of temperature, pH and metal ions on guaiacol oxidation of purified laccase from Leptographium qinlingensis. World J. Microbiol. Biotechnol. 30, 1285-1290.

Husain, M., Husain, Q., 2007. Applications of redox mediators in the treatment of organic pollutants by using oxidoreductive enzymes: a review. Crit. Rev. Environ. Sci. Technol. 38, 1-42.

Ikehata, K., Jodeiri Naghashkar, N., Gamal El-Din, M., 2006. Degradation of aqueous pharmaceuticals by ozonation and advanced oxidation processes: a review. Ozone Sci. Eng. 28, 353-414.

Jelic, A., Cruz-Morató, C., Marco-Urrea, E., Sarrà, M., Perez, S., Vicent, T., Petrović, M., Barcelo, D., 2012. Degradation of carbamazepine by Trametes versicolor in an air pulsed fluidized bed bioreactor and identification of intermediates. Water Res. 46, 955-964.

Ji, C., Hou, J., Chen, V., 2016a. Cross-linked carbon nanotubes based biocatalytic membranes for micro-pollutants degradation: performance, stability, and regeneration. J. Membr. Sci.

Ji, C., Hou, J., Wang, K., Zhang, Y., Chen, V., 2016b. Biocatalytic degradation of carbamazepine with immobilized laccase-mediator membrane hybrid reactor. J. Membr. Sci. 502, 11-20.

Jochems, P., Satyawali, Y., Diels, L., Dejonghe, W., 2011. Enzyme immobilization on in polymeric membranes: status, challenges and perspectives in biocatalytic membrane reactors (BMRs). Green Chem. 13, 1609-1623.

Joutey, N.T., Bahafid, W., Sayel, H., El Ghachtouli, N., Chamy, R., Rosenkranz, F., 2013. Biodegradation: Involved Microorganisms and Genetically Engineered Microorganisms, Biodegradation-life of science. InTech, Rijeka 289320.

Kang, S.-I., Kang, S.-Y., Hur, H.-G., 2008. Identification of fungal metabolites of anticonvulsant drug carbamazepine. Appl. Microbiol. Biotechnol. 79, 663.

Kanwar, L., Goswami, P., 2002. Isolation of a Pseudomonas lipase produced in pure hydrocarbon substrate and its application in the synthesis of isoamyl acetate using membrane-immobilised lipase. Enzyme Microb. Technol. 31, 727-735.

Kersten, P.J., 1990. Glyoxal oxidase of Phanerochaete chrysosporium: its characterization and activation by lignin peroxidase. Proc. Natl. Acad. Sci. 87, 2936-2940.

Kim, Y.J., Nicell, J.A., 2006. Laccase-catalysed oxidation of aqueous triclosan. J. Chem. Technol. Biotechnol. 81, 1344-1352.

Kim, S.D., Cho, J., Kim, I.S., Vanderford, B.J., Snyder, S.A., 2007. Occurrence and removal of pharmaceuticals and endocrine disruptors in South Korean surface, drinking, and waste waters. Water Res. 41, 1013-1021.

Kosjek, T., Andersen, H.R., Kompare, B., Ledin, A., Heath, E., 2009. Fate of carbamazepine during water treatment. Environ. Sci. Technol. 43, 6256-6261.

Kumar, V.V., Cabana, H., 2016. Towards high potential magnetic biocatalysts fo on-demand elimination of pharmaceuticals. Bioresour. Technol. 200, 81-89.

Kümmerer, K., 2008. Pharmaceuticals in the Environment-a Brief Summary, Pharmaceuticals in the Environment. Springer, 3-21.

Lagesson, A., Fahlman, J., Brodin, T., Fick, J., Jonsson, M., Byström, P., Klaminder, J., 2016. Bioaccumulation of five pharmaceuticals at multiple trophic levels in an aquatic food web - insights from a field experiment. Sci. Total Environ. 568 , 208-215.

Li, X., de Toledo, R.A., Wang, S., Shim, H., 2015. Removal of carbamazepine and naproxen by immobilized Phanerochaete chrysosporium under non-sterile condition. New Biotechnol. 32, 282-289.

Lienert, J., Güdel, K., Escher, B.I., 2007. Screening method for ecotoxicological hazard assessment of 42 pharmaceuticals considering human metabolism and excretory routes. Environ. Sci. Technol. 41, 4471-4478. 
Llorca, M., Rodríguez-Mozaz, S., Couillerot, O., Panigoni, K., de Gunzburg, J., Bayer, S., Czaja, R., Barceló, D., 2015. Identification of new transformation products during enzymatic treatment of tetracycline and erythromycin antibiotics at laboratory scale by an on-line turbulent flow liquid-chromatography coupled to a high resolution mass spectrometer LTQ-Orbitrap. Chemosphere 119, 90-98.

Lloret, L., Eibes, G., Lú-Chau, T.A., Moreira, M.T., Feijoo, G., Lema, J.M., 2010. Laccase-catalyzed degradation of anti-inflammatories and estrogens. Biochem. Eng. J. 51, 124-131.

Lloret, L., Eibes, G., Feijoo, G., Moreira, M., Lema, J.M., 2012a. Continuous biotransformation of estrogens by laccase in an enzymatic membrane reactor. Chem. Eng. 27.

Lloret, L., Hollmann, F., Eibes, G., Feijoo, G., Moreira, M., Lema, J., 2012b. Immobilisation of laccase on Eupergit supports and its application for the removal of endocrine disrupting chemicals in a packed-bed reactor. Biodegradation 23, 373-386.

Lloret, L., Eibes, G., Moreira, M., Feijoo, G., Lema, J., 2013. On the use of a high-redox potential laccase as an alternative for the transformation of non-steroidal anti-inflammatory drugs (NSAIDs). J. Mol. Catal. B Enzym. 97, 233-242.

Lonappan, L., Brar, S.K., Das, R.K., Verma, M., Surampalli, R.Y., 2016. Diclofenac and its transformation products: environmental occurrence and toxicity-A review. Environ. Int. 96, 127-138

Lonappan, L., Rouissi, T., Laadila, M.A., Brar, S.K., Hernández-Galán, L., Verma, M., Surampalli, R.Y., 2017. Agro-Industrial Produced Laccase for Degradation of Diclofenac and Identification of Transformation Products. ACS Sustainable Chemistry \& Engineering.

Lu, J., Huang, Q., 2009. Removal of acetaminophen using enzyme-mediated oxidative coupling processes: II. Cross-coupling with natural organic matter. Environ. Sci. Technol. 43, 7068-7073.

Lu, J., Huang, Q., Mao, L., 2009. Removal of acetaminophen using enzyme-mediated oxidative coupling processes: I. Reaction rates and pathways. Environ. Sci. Technol. 43, 7062-7067.

Lucas, D., Castellet-Rovira, F., Villagrasa, M., Badia-Fabregat, M., Barceló, D., Vicent, T., Caminal, G., Sarrà, M., Rodríguez-Mozaz, S., 2018. The role of sorption processes in the removal of pharmaceuticals by fungal treatment of wastewater. Sci. Total Environ. 610-611, 1147-1153.

Lundell, T.K., Mäkelä, M.R., Hildén, K., 2010. Lignin-modifying enzymes in filamentous basidiomycetes-ecological, functional and phylogenetic review. J. basic Microbiol. 50, 5-20.

Majeau, J.-A., Brar, S.K., Tyagi, R.D., 2010. Laccases for removal of recalcitrant and emerging pollutants. Bioresour. Technol. 101, 2331-2350.

Marco-Urrea, E., Pérez-Trujillo, M., Vicent, T., Caminal, G., 2009. Ability of white-rot fungi to remove selected pharmaceuticals and identification of degradation products of ibuprofen by Trametes versicolor. Chemosphere 74, 765-772.

Marco-Urrea, E., Pérez-Trujillo, M., Blánquez, P., Vicent, T., Caminal, G., 2010a. Biodegradation of the analgesic naproxen by Trametes versicolor and identification of intermediates using HPLC-DAD-MS and NMR. Bioresour. Technol. 101, 2159-2166.

Marco-Urrea, E., Pérez-Trujillo, M., Cruz-Morató, C., Caminal, G., Vicent, T., 2010b. Degradation of the drug sodium diclofenac by Trametes versicolor pellets and identification of some intermediates by NMR. J. Hazard. Mater. 176, 836-842.

Marco-Urrea, E., Pérez-Trujillo, M., Cruz-Morató, C., Caminal, G., Vicent, T., 2010c White-rot fungus-mediated degradation of the analgesic ketoprofen and identification of intermediates by HPLC-DAD-MS and NMR. Chemosphere 78, 474-481.

Marco-Urrea, E., Radjenović, J., Caminal, G., Petrović, M., Vicent, T., Barceló, D., 2010d. Oxidation of atenolol, propranolol, carbamazepine and clofibric acid by a biological Fenton-like system mediated by the white-rot fungus Trametes versicolor. Water Res. 44, 521-532.

Margot, J., Bennati-Granier, C., Maillard, J., Blánquez, P., Barry, D.A., Holliger, C., 2013a. Bacterial versus fungal laccase: potential for micropollutant degradation. AMB Express 3, 63 .

Margot, J., Maillard, J., Rossi, L., Barry, D.A., Holliger, C., 2013b. Influence of treatment conditions on the oxidation of micropollutants by Trametes versicolor laccase. New Biotechnol. 30, 803-813.

Margot, J., Copin, P.-J., von Gunten, U., Barry, D.A., Holliger, C., 2015. Sulfamethoxazole and isoproturon degradation and detoxification by a laccase-mediator system: influence of treatment conditions and mechanistic aspects. Biochem. Eng. J. $103,47-59$

Martens, R., Wetzstein, H.G., Zadrazil, F., Capelari, M., Hoffmann, P., Schmeer, N. 1996. Degradation of the fluoroquinolone enrofloxacin by wood-rotting fungi. Appl. Environ. Microbiol. 62, 4206-4209.

Martínková, L., Kotik, M., Marková, E., Homolka, L., 2016. Biodegradation of phenolic compounds by Basidiomycota and its phenol oxidases: a review. Chemosphere 149, 373-382.

Mateo, C., Palomo, J.M., Fernandez-Lorente, G., Guisan, J.M., Fernandez-Lafuente, R., 2007. Improvement of enzyme activity, stability and selectivity via immobilization techniques. Enzyme Microb. Technol. 40, 1451-1463.

Mir-Tutusaus, J., Sarrà, M., Caminal, G., 2016. Continuous treatment of non-sterile hospital wastewater by Trametes versicolor: how to increase fungal viability by means of operational strategies and pretreatments. J. Hazard. Mater. 318, 561-570.
Mohapatra, D.P., Brar, S.K., Tyagi, R.D., Picard, P., Surampalli, R.Y., 2012. Carbamazepine in municipal wastewater and wastewater sludge: ultrafast quantification by laser diode thermal desorption-atmospheric pressure chemical ionization coupled with tandem mass spectrometry. Talanta 99, 247-255.

Mukhopadhyay, A., Dasgupta, A.K., Chakrabarti, K., 2015. Enhanced functionality and stabilization of a cold active laccase using nanotechnology based activation-immobilization. Bioresour. Technol. 179, 573-584.

Naghdi, M., Taheran, M., Brar, S.K., Kermanshahi-pour, A., Verma, M., Surampalli, R., 2017. Immobilized laccase on oxygen functionalized nanobiochars through mineral acids treatment for removal of carbamazepine. Sci. Total Environ.

Nair, R.R., Demarche, P., Agathos, S.N., 2013. Formulation and characterization of an immobilized laccase biocatalyst and its application to eliminate organic micropollutants in wastewater. New Biotechnol. 30, 814-823.

Nazaret, S., Aminov, R., 2013. Role and prevalence of antibiosis and the related resistance genes in the environment. Front. Microbiol. 5, 520-520.

Nghiem, L.D., Coleman, P.J., Espendiller, C., 2010. Mechanisms underlying the effects of membrane fouling on the nanofiltration of trace organic contaminants. Desalination $250,682-687$.

Nguyen, L.N., Hai, F.I., Yang, S., Kang, J., Leusch, F.D., Roddick, F., Price, W.E., Nghiem, L.D., 2013. Removal of trace organic contaminants by an MBR comprising a mixed culture of bacteria and white-rot fungi. Bioresour. Technol. 148 , 234-241.

Nguyen, L.N., Hai, F.I., Kang, J., Leusch, F.D., Roddick, F., Magram, S.F., Price, W.E., Nghiem, L.D., 2014a. Enhancement of trace organic contaminant degradation by crude enzyme extract from Trametes versicolor culture: effect of mediator type and concentration. J. Taiwan Inst. Chem. Eng. 45, 1855-1862.

Nguyen, L.N., Hai, F.I., Price, W.E., Leusch, F.D., Roddick, F., McAdam, E.J., Magram, S.F., Nghiem, L.D., 2014b. Continuous biotransformation of bisphenol A and diclofenac by laccase in an enzymatic membrane reactor. Int. Biodeterior. Biodegrad. 95, 25-32.

Nguyen, L.N., Hai, F.I., Price, W.E., Leusch, F.D., Roddick, F., Ngo, H.H., Guo, W., Magram, S.F., Nghiem, L.D., 2014c. The effects of mediator and granular activated carbon addition on degradation of trace organic contaminants by an enzymatic membrane reactor. Bioresour. Technol. 167, 169-177.

Nguyen, L.N., Hai, F.I., Yang, S., Kang, J., Leusch, F.D.L., Roddick, F., Price, W.E., Nghiem, L.D., 2014d. Removal of pharmaceuticals, steroid hormones, phytoestrogens, UV-filters, industrial chemicals and pesticides by Trametes versicolor: role of biosorption and biodegradation. Int. Biodeterior. Biodegrad. 88, 169-175.

Nguyen, L.N., Hai, F.I., Price, W.E., Kang, J., Leusch, F.D., Roddick, F., van de Merwe, J.P., Magram, S.F., Nghiem, L.D., 2015. Degradation of a broad spectrum of trace organic contaminants by an enzymatic membrane reactor: complementary role of membrane retention and enzymatic degradation. Int. Biodeterior. Biodegrad. $99,115-122$.

Nguyen, L.N., Hai, F.I., Dosseto, A., Richardson, C., Price, W.E., Nghiem, L.D., 2016a. Continuous adsorption and biotransformation of micropollutants by granular activated carbon-bound laccase in a packed-bed enzyme reactor. Bioresour. Technol. 210, 108-116.

Nguyen, L.N., van de Merwe, J.P., Hai, F.I., Leusch, F.D., Kang, J., Price, W.E., Roddick, F., Magram, S.F., Nghiem, L.D., 2016b. Laccase-syringaldehyde-mediated degradation of trace organic contaminants in an enzymatic membrane reactor: removal efficiency and effluent toxicity. Bioresour. Technol. 200, 477-484.

Paiva, A.L., Balcao, V.M., Malcata, F.X., 2000. Kinetics and mechanisms of reactions catalyzed by immobilized lipases 访. Enzyme Microb. Technol. 27, 187-204.

Parshikov, I.A., Freeman, J.P., Lay, J.O., Beger, R.D., Williams, A.J., Sutherland, J.B., 1999. Regioselective transformation of ciprofloxacin to N-acetylciprofloxacin by the fungus Mucor ramannianus. FEMS Microbiol. Lett. 177, 131-135.

Parshikov, I.A., Freeman, J.P., Lay, J.O., Beger, R.D., Williams, A.J., Sutherland, J.B., 2000. Microbiological transformation of enrofloxacin by the fungus Mucor ramannianus. Appl. Environ. Microbiol. 66, 2664-2667.

Parshikov, I., Heinze, T., Moody, J., Freeman, J., Williams, A., Sutherland, J., 2001. The fungus Pestalotiopsis guepini as a model for biotransformation of ciprofloxacin and norfloxacin. Appl. Microbiol. Biotechnol. 56, 474-477.

Parshikov, I.A., Moody, J.D., Freeman, J.P., Lay Jr., J.O., Williams, A.J., Heinze, T.M., Sutherland, J.B., 2002. Formation of conjugates from ciprofloxacin and norfloxacin in cultures of Trichoderma viride. Mycologia 94, 1-5.

Parshikov, I.A., Miriyala, B., Muraleedharan, K.M., Illendula, A., Avery, M.A., Williamson, J.S., 2005. Biocatalysis of the antimalarial artemisinin by mucor ramannianus. Strains. Pharm. Biol. 43, 579-582.

Piccolo, A., Cozzolino, A., Conte, P., Spaccini, R., 2000. Polymerization of humic substances by an enzyme-catalyzed oxidative coupling. Naturwissenschaften 87 391-394.

Pointing, S., 2001. Feasibility of bioremediation by white-rot fungi. Appl. Microbiol. Biotechnol. 57, 20-33.

Prieto, A., Möder, M., Rodil, R., Adrian, L., Marco-Urrea, E., 2011. Degradation of the antibiotics norfloxacin and ciprofloxacin by a white-rot fungus and identification of degradation products. Bioresour. Technol. 102, 10987-10995.

Purich, D.L., 2010. Enzyme Kinetics: Catalysis and Control: a Reference of Theory and Best-practice Methods. Elsevier. 
Quaratino, D., Federici, F., Petruccioli, M., Fenice, M., D'Annibale, A., 2007. Production, purification and partial characterisation of a novel laccase from the white-rot fungus Panus tigrinus CBS 577.79. Antonie Leeuwenhoek 91, 57-69.

Radjenović, J., Petrović, M., Ventura, F., Barceló, D., 2008. Rejection of pharmaceuticals in nanofiltration and reverse osmosis membrane drinking water treatment. Water Res. 42, 3601-3610.

Rahmani, K., Faramarzi, M.A., Mahvi, A.H., Gholami, M., Esrafili, A., Forootanfar, H., Farzadkia, M., 2015. Elimination and detoxification of sulfathiazole and sulfamethoxazole assisted by laccase immobilized on porous silica beads. Int. Biodeterior. Biodegrad. 97, 107-114.

Rios, G., Belleville, M., Paolucci, D., Sanchez, J., 2004. Progress in enzymatic membrane reactors-a review. J. Membr. Sci. 242, 189-196.

Rodarte-Morales, A., Feijoo, G., Moreira, M., Lema, J., 2011. Degradation of selected pharmaceutical and personal care products (PPCPs) by white-rot fungi. World J. Microbiol. Biotechnol. 27, 1839-1846.

Rodarte-Morales, A., Feijoo, G., Moreira, M., Lema, J., 2012a. Biotransformation of three pharmaceutical active compounds by the fungus Phanerochaete chrysosporium in a fed batch stirred reactor under air and oxygen supply. Biodegradation 23, $145-156$.

Rodarte-Morales, A., Feijoo, G., Moreira, M., Lema, J., 2012b. Operation of stirred tank reactors (STRs) and fixed-bed reactors (FBRs) with free and immobilized Phanerochaete chrysosporium for the continuous removal of pharmaceutical compounds. Biochem. Eng. J. 66, 38-45.

Rodarte-Morales, A.I., Feijoo, G., Moreira, M.T., Lema, J.M., 2012c. Evaluation of two operational regimes: fed-batch and continuous for the removal of pharmaceuticals in a fungal stirred tank reactor. Chem. Eng. 27.

Rodgers, C.J., Blanford, C.F., Giddens, S.R., Skamnioti, P., Armstrong, F.A., Gurr, S.J., 2010. Designer laccases: a vogue for high-potential fungal enzymes?. Trends Biotechnol. 28, 63-72

Rodríguez-Rodríguez, C.E., Marco-Urrea, E., Caminal, G., 2010. Naproxen degradation test to monitor Trametes versicolor activity in solid-state bioremediation processes. J. Hazard. Mater. 179, 1152-1155.

Rodríguez-Rodríguez, C.E., Jelić, A., Llorca, M., Farré, M., Caminal, G., Petrović, M., Barceló, D., Vicent, T., 2011. Solid-phase treatment with the fungus Trametes versicolor substantially reduces pharmaceutical concentrations and toxicity from sewage sludge. Bioresour. Technol. 102, 5602-5608.

Rodríguez-Rodríguez, C.E., García-Galán, M.J., Blánquez, P., Díaz-Cruz, M.S., Barceló, D., Caminal, G., Vicent, T., 2012. Continuous degradation of a mixture of sulfonamides by Trametes versicolor and identification of metabolites from sulfapyridine and sulfathiazole. J. Hazard. Mater. 213, 347-354.

Rodrıguez, E., Nuero, O., Guillén, F., Martınez, A., Martınez, M., 2004. Degradation of phenolic and non-phenolic aromatic pollutants by four Pleurotus species: the role of laccase and versatile peroxidase. Soil Biol. Biochem. 36, 909-916.

Rosal, R., Rodríguez, A., Perdigón-Melón, J.A., Petre, A., García-Calvo, E., Gómez, M.J., Agüera, A., Fernández-Alba, A.R., 2010. Occurrence of emerging pollutants in urban wastewater and their removal through biological treatment followed by ozonation. Water Res. 44, 578-588.

Rouches, E., Herpoël-Gimbert, I., Steyer, J., Carrere, H., 2016. Improvement of anaerobic degradation by white-rot fungi pretreatment of lignocellulosic biomass: a review. Renewable Sustainable Energy Rev. 59, 179-198.

Routledge, E.J., Sumpter, J.P., 1996. Estrogenic activity of surfactants and some of their degradation products assessed using a recombinant yeast screen. Environ. Toxicol. Chem. 15, 241-248.

Sanchez Marcano, J.G., Tsotsis, T.T., 2004. Membrane Bioreactors, Catalytic Membranes and Membrane Reactors. Wiley-VCH Verlag GmbH \& Co. KGaA, $133-168$.

Sahar, E., Ernst, M., Godehardt, M., Hein, A., Herr, J., Kazner, C., Melin, T., Cikurel, H., Aharoni, A., Messalem, R., Brenner, A., Jekel, M., 2011. Comparison of two treatments for the removal of selected organic micropollutants and bulk organic matter: conventional activated sludge followed by ultrafiltration versus membrane bioreactor. Water Sci. Technol. 63, 733-740.

Sakaki, K., Giorno, L., Drioli, E., 2001. Lipase-catalyzed optical resolution of racemic naproxen in biphasic enzyme membrane reactors. J. Membr. Sci. 184, 27-38.

Sampaio, L.M., Padrão, J., Faria, J., Silva, J.P., Silva, C.J., Dourado, F., Zille, A., 2016. Laccase immobilization on bacterial nanocellulose membranes: antimicrobial, kinetic and stability properties. Carbohydr. Polym. 145, 1-12.

Sathishkumar, P., Chae, J.-C., Unnithan, A.R., Palvannan, T., Kim, H.Y., Lee, K.-J., Cho, M., Kamala-Kannan, S., Oh, B.-T., 2012. Laccase-poly (lactic-co-glycolic acid)(PLGA) nanofiber: highly stable, reusable, and efficacious for the transformation of diclofenac. Enzyme Microb. Technol. 51, 113-118.

Sathishkumar, P., Mythili, A., Hadibarata, T., Jayakumar, R., Kanthimathi, M., Palvannan, T., Ponraj, M., Salim, M.R., Yusoff, A.R.M., 2014. Laccase mediated diclofenac transformation and cytotoxicity assessment on mouse fibroblast 3T3-L1 preadipocytes. RSC Adv. 4, 11689-11697.

Schwarz, J., Aust, M.-O., Thiele-Bruhn, S., 2010. Metabolites from fungal laccase-catalysed transformation of sulfonamides. Chemosphere 81, 1469-1476.

Senthivelan, T., Kanagaraj, J., Panda, R., 2016. Recent trends in fungal laccase for various industrial applications: an eco-friendly approach-A review. Biotechnol. Bioprocess Eng. 21.
Sheldon, R.A., 2007. Enzyme immobilization: the quest for optimum performance. Adv. Synthesis Catal. 349, 1289-1307.

Shi, L., Ma, F., Han, Y., Zhang, X., Yu, H., 2014. Removal of sulfonamide antibiotics by oriented immobilized laccase on Fe $3 \mathrm{O} 4$ nanoparticles with natural mediators. J. Hazard. Mater. 279, 203-211.

Sondhi, S., Sharma, P., Saini, S., Puri, N., Gupta, N., 2014. Purification and characterization of an extracellular, thermo-alkali-stable, metal tolerant laccase from Bacillus tequilensis SN4. PLoS One 9, e96951.

Spengler, P., Körner, W., Metzger, J.W., 2001. Substances with estrogenic activity in effluents of sewage treatment plants in southwestern Germany. 1. Chemical analysis. Environ. Toxicol. Chem. 20, 2133-2141.

Stackelberg, P.E., Gibs, J., Furlong, E.T., Meyer, M.T., Zaugg, S.D., Lippincott, R.L., 2007. Efficiency of conventional drinking-water-treatment processes in removal of pharmaceuticals and other organic compounds. Sci. Total Environ. 377, 255-272.

Stadlmair, L.F., Letzel, T., Drewes, J.E., Graßmann, J., 2017. Mass spectrometry based in vitro assay investigations on the transformation of pharmaceutical compounds by oxidative enzymes. Chemosphere 174, 466-477.

Strong, P., Claus, H., 2011. Laccase: a review of its past and its future in bioremediation. Crit. Rev. Environ. Sci. Technol. 41, 373-434.

Suárez, S., Carballa, M., Omil, F., Lema, J.M., 2008. How are pharmaceutical and personal care products (PPCPs) removed from urban wastewaters? Rev. Environ. Sci. Bio/Technology 7, 125-138.

Suda, T., Hata, T., Kawai, S., Okamura, H., Nishida, T., 2012. Treatment of tetracycline antibiotics by laccase in the presence of 1-hydroxybenzotriazole. Bioresour. Technol. 103, 498-501.

Sutar, R.S., Rathod, V.K., 2015a. Ultrasound assisted enzyme catalyzed degradation of Cetirizine dihydrochloride. Ultrason. sonochemistry $24,80-86$.

Sutar, R.S., Rathod, V.K., 2015b. Ultrasound assisted Laccase catalyzed degradation of Ciprofloxacin hydrochloride. J. Industrial Eng. Chem. 31, 276-282.

Sutar, R.S., Rathod, V.K., 2016. Ultrasound assisted enzymatic degradation of diclofenac sodium: optimization of process parameters and kinetics. J. Water Process Eng. 9, e1-e6.

Tadkaew, N., Hai, F.I., McDonald, J.A., Khan, S.J., Nghiem, L.D., 2011. Removal of trace organics by MBR treatment: the role of molecular properties. Water Res. 45, 2439-2451.

Taheran, M., Brar, S.K., Verma, M., Surampalli, R.Y., Zhang, T.C., Valéro, J.R., 2016. Membrane processes for removal of pharmaceutically active compounds (PhACs) from water and wastewaters. Sci. Total Environ. 547, 60-77.

Taheran, M., Naghdi, M., Brar, S.K., Knystautas, E.J., Verma, M., Surampalli, R.Y., 2017a. Covalent Immobilization of Laccase onto Nanofibrous Membrane for Degradation of Pharmaceutical Residues in Water. ACS Sustainable Chemistry \& Engineering.

Taheran, M., Naghdi, M., Brar, S.K., Knystautas, E.J., Verma, M., Surampalli, R.Y., 2017b. Degradation of chlortetracycline using immobilized laccase on Polyacrylonitrile-biochar composite nanofibrous membrane. Sci. Total Environ. 605-606, 315-321.

Tiwari, B., Sellamuthu, B., Ouarda, Y., Drogui, P., Tyagi, R.D., Buelna, G., 2017. Review on fate and mechanism of removal of pharmaceutical pollutants from wastewater using biological approach. Bioresour. Technol. 224, 1-12.

Touahar, I.E., Haroune, L., Ba, S., Bellenger, J.-P., Cabana, H., 2014. Characterization of combined cross-linked enzyme aggregates from laccase, versatile peroxidase and glucose oxidase, and their utilization for the elimination of pharmaceuticals. Sci. Total Environ. 481, 90-99.

Tran, N.H., Urase, T., Kusakabe, O., 2010. Biodegradation characteristics of pharmaceutical substances by whole fungal culture Trametes versicolor and its laccase. J. Water Environ. Technol. 8, 125-140.

Trusek-Holownia, A., Noworyta, A., 2007. An integrated process: ester synthesis in an enzymatic membrane reactor and water sorption. J. Biotechnol. 130, 47-56.

Turło, J., Turło, A., 2013. Application of mushroom cultures and isolated enzymes for biodegradation of organic environmental pollutants. Mil. Pharm. Med. 3, 27-36.

Van Leeuwen, J., Hu, Z., Yi, T., Pometto III, A., Jin, B., 2003. Kinetic model for selective cultivation of microfungi in a microscreen process for food processing wastewater treatment and biomass production. Eng. Life Sci. 23, 289-300.

Vergeynst, L., Haeck, A., De Wispelaere, P., Van Langenhove, H., Demeestere, K., 2015. Multi-residue analysis of pharmaceuticals in wastewater by liquid chromatography-magnetic sector mass spectrometry: method quality assessment and application in a Belgian case study. Chemosphere 119, S2-S8.

Wan, J., Zeng, G., Huang, D., Huang, C., Lai, C., Li, N., Wei, Z., Xu, P., He, X., Lai, M., 2015. The oxidative stress of Phanerochaete chrysosporium against lead toxicity. Appl. Biochem. Biotechnol. 175, 1981-1991.

Wang, J., Wang, S., 2016. Removal of pharmaceuticals and personal care products (PPCPs) from wastewater: a review. J. Environ. Manag. 182, 620-640.

Wang, J., Majima, N., Hirai, H., Kawagishi, H., 2012. Effective removal of endocrine-disrupting compounds by lignin peroxidase from the white-rot fungus Phanerochaete sordida YK-624. Curr. Microbiol. 64, 300-303.

Wen, X., Jia, Y., Li, J., 2009. Degradation of tetracycline and oxytetracycline by crude lignin peroxidase prepared from Phanerochaete chrysosporium-a white rot fungus. Chemosphere 75, 1003-1007. 
Wen, X., Jia, Y., Li, J., 2010. Enzymatic degradation of tetracycline and oxytetracycline by crude manganese peroxidase prepared from Phanerochaete chrysosporium. J. Hazard. Mater. 177, 924-928.

Weng, S.-S., Ku, K.-L., Lai, H.-T., 2012. The implication of mediators for enhancement of laccase oxidation of sulfonamide antibiotics. Bioresour. Technol. 113 , 259-264.

Weng, S.-S., Liu, S.-M., Lai, H.-T., 2013. Application parameters of laccase-mediator systems for treatment of sulfonamide antibiotics. Bioresour. Technol. 141 152-159.

Westerhoff, P., Moon, H., Minakata, D., Crittenden, J., 2009. Oxidation of organics in retentates from reverse osmosis wastewater reuse facilities. Water Res. 43, 3992-3998.

Wetzstein, H.-G., Schmeer, N., Karl, W., 1997. Degradation of the fluoroquinolone enrofloxacin by the brown rot fungus Gloeophyllum striatum: identification of metabolites. Appl. Environ. Microbiol. 63, 4272-4281.

Wetzstein, H.-G., Stadler, M., Tichy, H.-V., Dalhoff, A., Karl, W., 1999. Degradation of ciprofloxacin by basidiomycetes and identification of metabolites generated by the brown rot FungusGloeophyllum striatum. Appl. Environ. Microbiol. 65, $1556-1563$.

Wetzstein, H.-G., Schneider, J., Karl, W., 2006. Patterns of metabolites produced from the fluoroquinolone enrofloxacin by basidiomycetes indigenous to agricultural sites. Appl. Microbiol. Biotechnol. 71, 90-100.

Wong, D.W.S., 2009. Structure and action mechanism of ligninolytic enzymes. Appl. Biochem. Biotechnol. 157, 174-209.

Xu, F., 1996. Oxidation of phenols, anilines, and benzenethiols by fungal laccases: correlation between activity and redox potentials as well as halide inhibition. Biochemistry $35,7608-7614$.

Xu, J., Wang, Y., Hu, Y., Luo, G., Dai, Y., 2006. Immobilization of lipase by filtration into a specially designed microstructure in the CA/PTFE composite membrane. J. Mol. Catal. B Enzym. 42, 55-63.

Xu, R., Si, Y., Li, F., Zhang, B., 2015a. Enzymatic removal of paracetamol from aqueous phase: horseradish peroxidase immobilized on nanofibrous membranes. Environ. Sci. Pollut. Res. 22, 3838-3846.
Xu, R., Tang, R., Zhou, Q., Li, F., Zhang, B., 2015b. Enhancement of catalytic activity of immobilized laccase for diclofenac biodegradation by carbon nanotubes. Chem. Eng. J. 262, 88-95.

Yang, S., 2012. Removal of Micropollutants by a Fungus-augmented Membrane Bioreactor. University of Wollongong.

Yang, S., Hai, F.I., Nghiem, L.D., Nguyen, L.N., Roddick, F., Price, W.E., 2013a. Removal of bisphenol A and diclofenac by a novel fungal membrane bioreactor operated under non-sterile conditions. Int. Biodeterior. Biodegrad. 85, 483-490.

Yang, S., Hai, F.I., Nghiem, L.D., Price, W.E., Roddick, F., Moreira, M.T., Magram, S.F., 2013b. Understanding the factors controlling the removal of trace organic contaminants by white-rot fungi and their lignin modifying enzymes: a critical review. Bioresour. Technol. 141, 97-108.

Yang, S., Hai, F.I., Nghiem, L.D., Roddick, F., Price, W.E., 2013c. Removal of trace organic contaminants by nitrifying activated sludge and whole-cell and crude enzyme extract of Trametes versicolor. Water Sci. Technol. 67, 1216-1223.

Yujun, W., Jian, X., Guangsheng, L., Youyuan, D., 2008. Immobilization of lipase by ultrafiltration and cross-linking onto the polysulfone membrane surface. Bioresour. Technol. 99, 2299-2303.

Zhan, Y., Wu, Y., Xu, F., Bai, Y., Guan, Y., Williamson, J.S., Liu, B., 2017. A novel dihydroxylated derivative of artemisinin from microbial transformation. Fitoterapia 120, 93-97.

Zhang, Y., Geißen, S.-U., 2010. In vitro degradation of carbamazepine and diclofenac by crude lignin peroxidase. J. Hazard. Mater. 176, 1089-1092.

Zhang, Y., Geißen, S.-U., 2012. Elimination of carbamazepine in a non-sterile fungal bioreactor. Bioresour. Technol. 112, 221-227.

Zouari-Mechichi, H., Mechichi, T., Dhouib, A., Sayadi, S., Martínez, A.T., Martínez, M.J., 2006. Laccase purification and characterization from Trametes trogii isolated in Tunisia: decolorization of textile dyes by the purified enzyme. Enzyme Microb. Technol. 39, 141-148.

Zuehlke, S., Duennbier, U., Lesjean, B., Gnirss, R., Buisson, H., 2006. Long-term comparison of trace organics removal performances between conventional and membrane activated sludge processes. Water Environ. Res. 78, 2480-2486. 